\title{
A census of semisymmetric cubic graphs on up to 768 vertices
}

\author{
Marston Conder ${ }^{1} \cdot$ Aleksander Malnič ${ }^{2}$. \\ Dragan Marušič² • Primož Potočnik ${ }^{3}$
}

Received: November 29, 2004 / Accepted: September 27, 2005

(C) Springer Science + Business Media, Inc. 2006

\begin{abstract}
A list is given of all semisymmetric (edge- but not vertex-transitive) connected finite cubic graphs of order up to 768. This list was determined by the authors using Goldschmidt's classification of finite primitive amalgams of index $(3,3)$, and a computer algorithm for finding all normal subgroups of up to a given index in a finitely-presented group. The list includes several previously undiscovered graphs. For each graph in the list, a significant amount of information is provided, including its girth and diameter, the order of its automorphism group, the order and structure of a minimal edge-transitive group of automorphisms, its Goldschmidt type, stabiliser partitions, and other details about its quotients and covers. A summary of all known infinite families of semisymmetric cubic graphs is also given, together with explicit rules for their construction, and members of the list are identified with these. The special case of those graphs having $K_{1,3}$ as a normal quotient is investigated in detail.
\end{abstract}

Keywords Semisymmetric graphs $\cdot$ Edge-transitive graphs $\cdot$ Amalgams

\section{Introduction}

A bipartite regular graph is called semisymmetric if its automorphism group has a single orbit on edges but two orbits on vertices (the two parts of the bipartition).

\footnotetext{
${ }^{1}$ Supported in part by N.Z. Marsden Fund (grant no. UOA 124) and N.Z. Centres of Research Excellence Fund (grant no. UOA 201)

${ }^{2}$ Supported in part by "Ministrstvo za šolstvo, znanost in šport Slovenije", research program no. 101-506.

${ }^{3}$ Supported in part by research projects no. Z1-4186-0101 and no. Z1-3124-0101. The fourth author would like to thank the University of Auckland for hospitality during his visit there in 2003.
}

M. Conder $(\square)$

Department of Mathematics, University of Auckland, Private Bag 92019 Auckland, New Zealand

A. Malnič $\cdot$ P. Potočnik $(\bowtie)$

IMFM, Oddelek za matematiko, Univerza v Ljubljani, Jadranska 19, 1111 Ljubljana, Slovenija

D. Marušič

University of Primorska, Cankarjeva 5, 6000, Koper, Slovenia 
More generally, given a graph $X$, let $V(X), E(X), A(X)$ and Aut $X$ be the vertex set, the edge set, the arc set and the automorphism group of $X$, respectively. If a subgroup $G$ of Aut $X$ acts transitively on $V(X), E(X)$ and $A(X)$, then $X$ is said to be $G$-vertex-transitive, $G$-edge-transitive and $G$-arc-transitive (or $G$-symmetric), respectively. It is easily seen that a graph $X$ which is $G$-edge- but not $G$-vertex-transitive is necessarily bipartite, with the two parts of the bipartition coinciding with the orbits of $G$. In particular, if $X$ is a regular graph, then these two parts have equal cardinalities, and such a graph is then referred to as being $G$-semisymmetric. In the case where $G=$ Aut $X$ the symbol $G$ may be omitted from the definitions above, so that $X$ is called symmetric if it is regular and (Aut $X$ )-arc-transitive, and semisymmetric if it is regular and (Aut $X$ )-edge-transitive but not (Aut $X$ )-vertex-transitive.

The study of semisymmetric graphs was initiated by Folkman [15], who gave a construction of several infinite families of such graphs and posed a number of open problems which spurred the interest in this topic. Several papers followed; see [2, 3, 13, 14, 24, 25, 26, 27 , $33,35]$.

This article is devoted to the case of cubic (trivalent) semisymmetric graphs. Motivated by the Foster census of arc-transitive cubic graphs on up to 512 vertices [4], the complete determination of all such graphs on up to 768 vertices [7], and the extended Foster census of these up to 998 vertices [6] (incomplete in the range 770-998), we give a complete list of all connected finite semisymmetric cubic graphs of order up to 768 . This list contains several previously undiscovered graphs; the third smallest graph on the list, on 112 vertices, is the subject of a separate paper [8].

The construction of this list of semisymmetric cubic graphs was achieved using Goldschmidt's classification [20] of finite primitive amalgams of index (3, 3). Roughly speaking, an amalgam of index $(3,3)$ is a pair of groups $P_{1}$ and $P_{2}$ having a common subgroup of index 3 in both, such as the stabilisers in Aut $X$ of the vertices $u$ and $v$ of some edge $\{u, v\}$ in a cubic graph $X$. Goldschmidt's classification implies that the automorphism group of every edge-transitive cubic graph is obtainable as a homomorphic image of the universal completion of one of 15 finite amalgams, denoted by $G_{1}, G_{1}^{1}, G_{1}^{2}, G_{1}^{3}, G_{2}, G_{2}^{1}, G_{2}^{2}, G_{2}^{3}, G_{2}^{4}, G_{3}, G_{3}^{1}$, $G_{4}, G_{4}^{1}, G_{5}$ and $G_{5}^{1}$. Each universal completion is essentially an amalgamated free product of the two subgroups $P_{1}$ and $P_{2}$ (with their common subgroup of index 3 amalgamated), and hence has a finite presentation in terms of generators and defining relations. By abuse of notation we use the same symbols $\left(G_{1}, G_{1}^{1}\right.$, and so on) to denote these 15 finitely-presented groups, and call each such symbol the Goldschmidt type of the corresponding amalgam.

Note that in all of these 15 finite primitive amalgams of index $(3,3)$, the orders of the two subgroups $P_{1}$ and $P_{2}$ are at most $384=3 \cdot 2^{7}$, and it follows that in the automorphism group of any finite connected edge-transitive cubic graph $X$, the stabiliser of any vertex has order $3 \cdot 2^{s-1}$ for some $s \leq 8$. A now classical theorem of Tutte [37] states that $s \leq 5$ in the special case where $X$ is symmetric.

Adopting the same approach as taken in [7] to determine all connected finite symmetric cubic graphs, we used a computerised algorithm for finding all normal subgroups of up to a certain index in each of the 15 finitely-presented groups. In fact again we used the "mixed" version of this algorithm (as explained in [7]), involving coset enumeration over the subgroup $P_{1}$ in each case, so that the upper bound on the index was taken as $768 / 2=384$ in each case, in order to obtain all finite homomorphic images representable as an edge-transitive group of automorphisms of some connected finite cubic graph. A more detailed description of this algorithm and unix implementations of it are available from Peter Dobcsányi [12]. The MAGMA system was used to construct and analyse the resulting graphs, and to determine vertex-transitivity (and hence distinguish between symmetric and semisymmetric cases). 
This article is organised as follows. Background material on amalgams (including a table showing the 15 types of primitive amalgam of index $(3,3)$ ) is given in Section 2, followed by some basic theory of quotients and covering projections in Section 3. A brief summary of all known semisymmetric cubic graphs (together with a number of new constructions of such graphs) is given in Section 4. In Section 5, a more detailed analysis of one of these families is undertaken, namely of those semisymmetric cubic graphs having $K_{1,3}$ as a normal quotient. The list itself is presented in Section 7, including detailed information on several graph- and group-theoretic parameters for each of the graphs, and is preceded by some explanation in Section 8. Finally in Section 6 we show how some of the graphs in the list can be obtained as covers of smaller symmetric cubic graphs using voltage constructions.

For general background on graphs and groups, as well as for the concepts and notation not defined here, we refer the reader to $[10,19,21,38]$.

\section{Amalgams of index $(3,3)$}

Following [20], an amalgam is a pair $\left(\phi_{1}, \phi_{2}\right)$ of group monomorphisms $\phi_{1}: B \rightarrow P_{1}$ and $\phi_{2}: B \rightarrow P_{2}$ with the same domain $B$. The amalgam is said to be finite if the codomains $P_{1}$ and $P_{2}$ are finite. The pair $\left(\left|P_{1}: \operatorname{im} \phi_{1}\right|,\left|P_{2}: \operatorname{im} \phi_{2}\right|\right)$ of indices is called the index of the amalgam. The amalgam is said to be primitive if for each $i \in\{1,2\}$ and for each non-trivial subgroup $K$ of $B$ such that $\phi_{3-i}(K)$ is normal in $P_{3-i}$, the normaliser of $\phi_{i}(K)$ in $P_{i}$ is $\phi_{i}(B)$. Two amalgams $\left(\phi_{1}: B \rightarrow P_{1}, \phi_{2}: B \rightarrow P_{2}\right)$ and $\left(\phi_{1}^{\prime}: B^{\prime} \rightarrow P_{1}^{\prime}, \phi_{2}^{\prime}: \mathrm{B}^{\prime} \rightarrow P_{2}^{\prime}\right)$ are isomorphic if there exist group isomorphisms $\alpha: B \rightarrow \mathrm{B}^{\prime}, \alpha_{1}: P_{1} \rightarrow P_{1}^{\prime}$ and $\alpha_{2}: P_{2} \rightarrow P_{2}^{\prime}$, making the obvious diagrams commutative.

A completion of the amalgam $\left(\phi_{1}, \phi_{2}\right)$ is a pair of group homomorphisms $\left(\psi_{1}: P_{1} \rightarrow G\right.$, $\psi_{2}: P_{2} \rightarrow G$ ) into the same group $G$, such that $\psi_{1} \circ \phi_{1}=\psi_{2} \circ \phi_{2}$, and such that the images of $\psi_{1}$ and $\psi_{2}$ generate $G$. If $\psi_{1}$ and $\psi_{2}$ are monomorphisms, then the completion is said to be faithful. The completion is called finite if the group $G$ is finite. For every amalgam $\left(\phi_{1}: B \rightarrow\right.$ $\left.P_{1}, \phi_{2}: B \rightarrow P_{2}\right)$, there is a so-called universal completion $\left(\psi_{1}^{\circ}: P_{1} \rightarrow G^{\circ}, \psi_{2}^{\circ}: P_{2} \rightarrow G^{\circ}\right)$ with the following defining property: for every completion $\left(\psi_{1}: P_{1} \rightarrow G, \psi_{2}: P_{2} \rightarrow G\right)$ of $\left(\phi_{1}, \phi_{2}\right)$ there exists a group homomorphism $\alpha: G^{\circ} \rightarrow G$, such that $\psi_{i}=\alpha \circ \psi_{i}^{\circ}$ for $i=1,2$. The group $G^{\circ}$ is usually called the amalgamated product of $P_{1}$ and $P_{2}$ (with amalgamated subgroup $B$ ), and denoted by $P_{1} *_{B} P_{2}$. In particular, every faithful finite completion can be obtained from the universal completion by taking as the kernel of $\alpha$ some normal subgroup of finite index in $G^{\circ}$ that intersects $\psi_{i}^{\circ}\left(P_{i}\right)$ trivially for $i=1,2$.

Now let $\left(\psi_{1}: P_{1} \rightarrow G, \psi_{2}: P_{2} \rightarrow G\right)$ be a faithful, finite completion of a finite amalgam $\left(\phi_{1}: B \rightarrow P_{1}, \phi_{2}: B \rightarrow P_{2}\right)$. By abuse of notation, we may identify the groups $B, P_{1}$ and $P_{2}$ with their images in $G$. We then define the graph of the completion as the bipartite graph $X=X\left(G, P_{1}, P_{2}\right)$ with vertex set $V$ the union of the two (right) coset spaces $\left[G: P_{1}\right]$ and [G: $P_{2}$ ], and with adjacency given by $P_{1} g \sim P_{2} h$ if and only if $P_{1} g \cap P_{2} h$ is non-empty. It is easy to see that the group $G$ acts (via right multiplication) as a group of automorphisms of $X$, and that this action is transitive on edges but not on vertices; moreover, this action is faithful if and only if $\operatorname{core}_{G}(B)=\bigcap_{g \in G} g^{-1} B g=1$, which is equivalent to the primitivity of the amalgam $\left(\phi_{1}, \phi_{2}\right)$.

Note that the valence of any vertex $P_{i} g$ is $\left|P_{i}: B\right|$, for $i=1,2$. This implies that the graph of a faithful finite completion of a finite amalgam of index $(3,3)$ is a cubic $G$-semisymmetric graph, with stabilisers of any two adjacent vertices isomorphic to $P_{1}$ and $P_{2}$ in some order. The converse of this fact together with the classification of all finite primitive amalgams of index $(3,3)$ achieved in $[20$, Theorem $A]$ is crucial for our analysis of semisymmetric cubic graphs: 
Proposition 2.1. Let $X$ be a finite cubic G-semisymmetric graph other than $K_{3,3}$. Let $\{u, v\}$ be any edge of $X$, and let $G_{u}$ and $G_{v}$ be the stabilisers in $G$ of $u$ and $v$ respectively. Then the pair $\left(\psi_{1}: G_{u} \rightarrow G, \psi_{2}: G_{v} \rightarrow G\right)$ of inclusion maps is a faithful finite completion of the finite primitive amalgam $\left(\phi_{1}, \phi_{2}\right)$, of index $(3,3)$, given by the inclusion maps of the intersection $G_{u} \cap G_{v}$ into $G_{u}$ and $G_{v}$ respectively, and $X$ is isomorphic to the graph of this completion.

Theorem 2.2. [20, Theorem A] There are precisely fifteen isomorphism classes of finite primitive amalgams of index $(3,3)$. The corresponding fifteen universal completions are given in Table 1.

\section{Quotients and coverings}

In order to facilitate easier reading, we give a short account on quotient projections, and regular covering projections in particular. (For a more extensive treatment of coverings, see $[23,31,32]$ for example.)

Quotients and coverings We say that two graph morphisms $f: X \rightarrow \bar{X}$ and $f^{\prime}: X^{\prime} \rightarrow$ $\bar{X}^{\prime}$ are isomorphic, written $f \cong f^{\prime}$, if there exist graph isomorphisms $\alpha: X \rightarrow X^{\prime}$ and $\bar{\alpha}: \bar{X} \rightarrow$ $\bar{X}^{\prime}$ such that $\bar{\alpha} \circ f=f^{\prime} \circ \alpha$. (We tacitly assume that graph morphisms are composed on the left, with composition written as $\circ$. However, graph automorphisms are occasionally composed on the right; this is indicated by juxtaposition, that is, by using no special sign for the composition.) If $X=X^{\prime}$ and $\alpha=$ id we call $f \cong f^{\prime}$ an equivalence below $X$ (or just equivalence from below). If $\bar{X}=\bar{X}^{\prime}$ and $\bar{\alpha}=$ id we call $f \cong f^{\prime}$ an equivalence above $\bar{X}$ (or just equivalence from above).

Let $X$ be a graph and $N \leq$ Aut $X$. The quotient graph $X_{N}$ has the vertex orbits $\{[v]$ : $v \in V(X)\}$ of $N$ as the vertex set, the arc orbits $\{[a]: a \in A(X)\}$ as the arc set (and hence the edge orbits $\{[e]: e \in E(X)\}$ as the edge set), and the adjacencies in $X_{N}$ are defined in a natural way so that the mappings $v \mapsto[v]$ and $a \mapsto[a]$ on vertices and arcs (and hence $e \mapsto[e]$ on edges) define a graph epimorphism $q_{N}: X \rightarrow X_{N}$, the quotient projection by the action of $N$. (Note that $X_{N}$ may have multiple edges, loops or even semiedges-a semiedge is created whenever two inverse arcs of $X$ are in the same orbit of $N$.) By abuse of terminology, any graph epimorphism $q: X \rightarrow \bar{X}$ which is equivalent below $X$ to a quotient projection $q_{N}: X \rightarrow X_{N}$ is also called a quotient projection and often identified with $q_{N}$-although a quotient projection might arise from several distinct groups; this fact is a source of many anomalies to be discussed further below.

The kernel of a quotient projection $q_{N}: X \rightarrow X_{N}$ is the largest subgroup $\operatorname{Ker} q_{N} \leq$ Aut $X$ having the same orbits as the group $N$. This group is uniquely defined and $\operatorname{Ker} q_{N}=\{\alpha \in$ Aut $\left.X \mid q_{N} \circ \alpha=q_{N}\right\}$. Thus, $q_{N}=q_{N^{\prime}}$ if and only if $\operatorname{Ker} q_{N}=\operatorname{Ker} q_{N^{\prime}}$ (for instance, we do have $q_{N}=q_{N^{\prime}}$ whenever $N \leq N^{\prime} \leq \operatorname{Ker} q_{N}$, but the converse might fail to be true). Also, two quotient projections $q_{N}$ and $q_{N^{\prime}}$ defined on a graph $X$ are isomorphic if and only if Ker $q_{N}$ and $\operatorname{Ker} q_{N^{\prime}}$ are conjugate subgroups of Aut $X$; in particular $q_{N}$ and $q_{N^{\prime}}$ are equivalent below $X$ if and only if $q_{N}=q_{N^{\prime}}$. Finally, $q_{N}$ is an isomorphism if and only if $N$ is the trivial group (because Aut $X$ acts faithfully on the set of arcs).

Let $X$ be a graph. We say that a group $N \leq$ Aut $X$ acts semiregularly on $X$ if it acts semiregularly on the vertex set $V(X)$ and on the arc set $A(X)$. The respective quotient projection $X \rightarrow X_{N}$ is called a regular $N$-covering projection and is usually denoted by $\wp_{N}$. If $N$ belongs to some specific class of groups such as cyclic, elementary abelian etc., then the respective regular covering covering projection is also called cyclic, elementary abelian etc. 
Table 1 Universal completions of finite primitive amalgams of index $(3,3)$

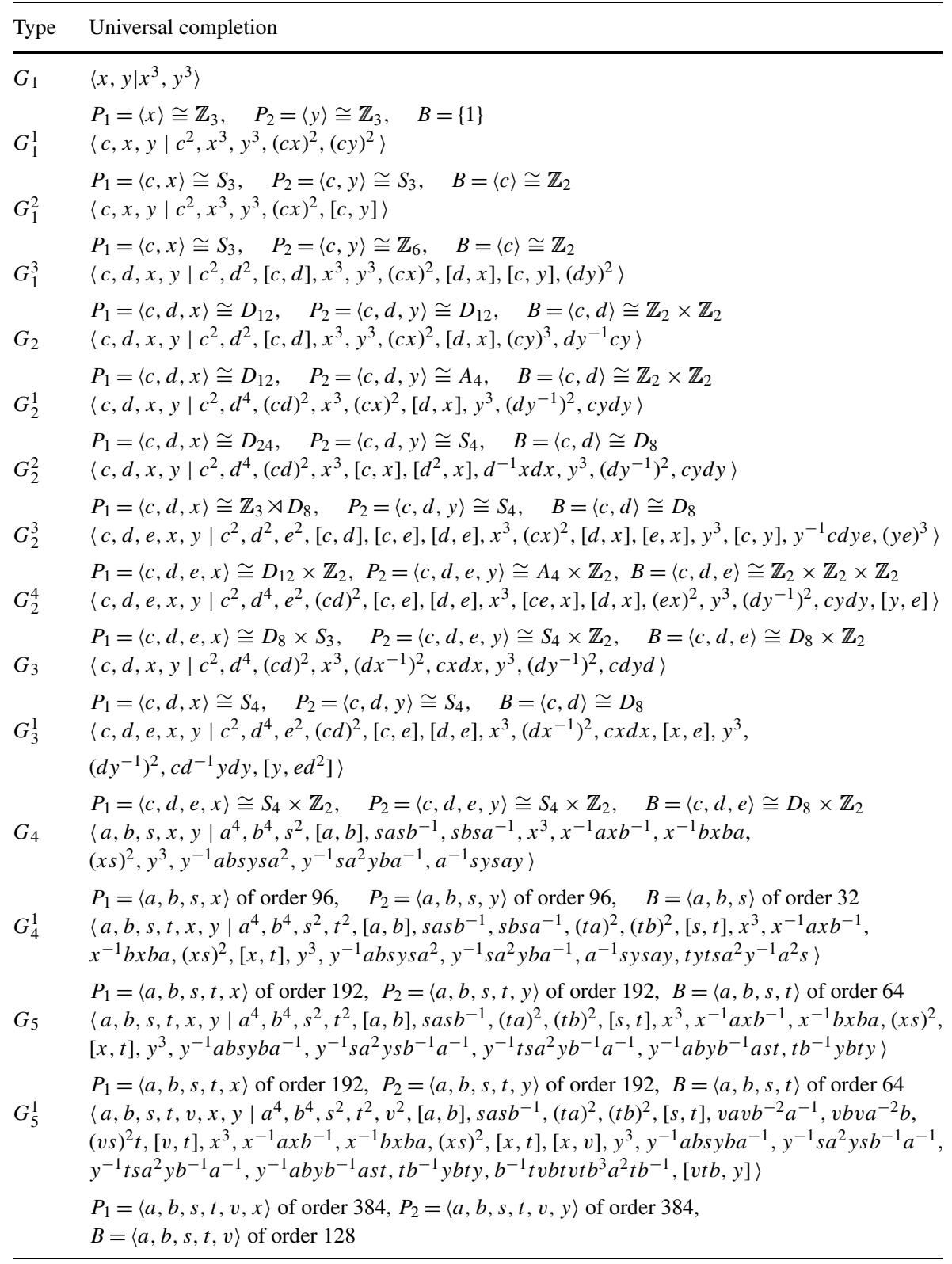

By abuse of terminology, any graph epimorphism $\wp: X \rightarrow \bar{X}$ which is equivalent below $X$ to a regular $N$-covering projection $\wp_{N}: X \rightarrow X_{N}$ is itself called a regular covering projection (and often identified with $\left.\wp_{N}\right)$. Note that the group CT $(\wp)=N=\operatorname{Ker} \wp$, also known as the group of covering transformations, is now uniquely determined by $\wp$. This is one of the many nice properties that regular covering projections share and which makes them rather distinguished among quotient projections in general. Another such (characteristic) property 
is the following: for each arc starting at $[v] \in V\left(X_{N}\right)$ and each vertex $u \in[v]$, there exists a unique arc in $X$ starting at $u$ which projects to the selected arc in $X_{N}$-this property extends to walks and it is known as the unique walk lifting property; in other words: a quotient projection is a regular covering projection if and only if it is valency preserving.

A regular covering projection $\wp: X \rightarrow \bar{X}$ can be reconstructed from $\bar{X}$, up to equivalence above $\bar{X}$, as follows. Consider $\mathrm{CT}(\wp)=N$ as an abstract group, now called the voltage group. In each vertex fibre $\wp^{-1}(\bar{v}), \bar{v} \in V(\bar{X})$, choose a vertex and label it by $1 \in N$. Then transfer the labelling by the action of $N$ (considered as a group of automorphisms of $X$ acting on the left) to all the vertices of $X$. Let $\bar{a} \in A(\bar{X})$. Then there exists an element $\zeta(\bar{a}) \in N$, called the voltage of $\bar{a}$, such that all arcs in the arc fibre $\wp^{-1}(\bar{a})$ over $\bar{a} \in A(\bar{X})$ have origins and termini labelled by $g$ and $g \zeta(\bar{a})$, where $g \in N$. Obviously, inverse arcs receive inverse voltages. The graph $\operatorname{Cov}(X ; \zeta)$ with vertex set $V(\bar{X}) \times N$ and edge set $E(\bar{X}) \times N=\{\{(\bar{u}, g),(\bar{v}, g \zeta(\bar{a})\} \mid \bar{a} \in$ $A(\bar{X})$ with origin $\bar{u}$ and terminus $\bar{v}, g \in N$ \} is isomorphic to $X$. Obviously, $N$ acts by left multiplication on the second coordinate as a group of automorphisms of $\operatorname{Cov}(\bar{X} ; \zeta)$, and gives rise to a regular covering projection $\wp_{\zeta}=\wp_{N}: \operatorname{Cov}(\bar{X} ; \zeta) \rightarrow \bar{X}$ which is equivalent above $\bar{X}$ to $\wp: X \rightarrow \bar{X}$. As an abstract voltage group, $N$ acts by right multiplication on itself as the set of labels. It is well known that the voltages can be assigned in such a way that the arcs of an arbitrary chosen spanning tree of $\bar{X}$ receive the trivial voltage.

Conversely, given a graph $\bar{X}$, an abstract group $N$, and a voltage assignment $\zeta: A(\bar{X}) \rightarrow N$ such that the inverse arcs receive inverse voltages, the construction of $\operatorname{Cov}(\bar{X} ; \zeta)$ as above gives a regular covering projection $\wp_{\zeta}=\wp_{N}: \operatorname{Cov}(\bar{X} ; \zeta) \rightarrow \bar{X}$. The only inconvenience with $\zeta$ being arbitrary is that the resulting derived graph might be disconnected. The additional "if and only if" condition which should be imposed on $\zeta$ such that $\operatorname{Cov}(\bar{X} ; \zeta)$ is connected, is well known: each element of $N$ should appear as the voltage of some closed walk. If we assume that the arcs of an arbitrarily chosen spanning tree carry the trivial voltage then the derived covering graph is connected if and only if the voltages of the "off-tree arcs" generate the group $N$.

Projecting and lifting automorphisms Let $H \leq$ Aut $X$. We say that $H$ projects along $q_{N}: X \rightarrow X_{N}$ if, for each $h \in H$, there exists an automorphism $\bar{h} \in$ Aut $X_{N}$ such that $\bar{h}$ 。 $q_{N}=q_{N} \circ h$, called the projection of $h$ along $q_{N}$. Note that if $\bar{h}$ exists then it is unique. So if $H$ projects then we have a naturally defined group $\bar{H} \leq$ Aut $X_{N}$, the projection of $H$ along $q_{N}$, and we have an induced group epimorphism $q_{N}^{*}: H \rightarrow \bar{H}$, taking $h \mapsto \bar{h}$. We say that $G \in$ Aut $X_{N}$ lifts along $q_{N}$ if there exists a group $H \leq$ Aut $X$ which projects to $G$, that is, $\bar{H}=G$. The largest group that projects onto $G$ is called the lift of $G$ along $q_{N}$ and is denoted by $\widetilde{G}$ (such a group is unique). In particular, the kernel $\operatorname{Ker} q_{N}$ is the lift of the trivial group.

If a group $H \leq$ Aut $X$ normalizes $N$, then $H$ projects along $q_{N}$, and the groups $H /(H \cap N)$ and $\bar{H}$ have the same permutation representation on $X_{N}$; but the action of $H /(H \cap N)$ might not be faithful. On the other hand, $H$ may project even if $H$ does not normalize $N$. In particular, $N$ might not be a normal subgroup of $\operatorname{Ker} q_{N}$. These anomalies are not present with regular covering projections, since $\operatorname{Ker} \wp_{N}=N$ in their case.

Proposition 3.1. Let $X$ be a graph and $N \leq$ Aut $X$. Then a group $H \leq$ Aut $X$ projects along $q_{N}: X \rightarrow X_{N}$ if and only if $H$ normalizes $\operatorname{Ker} q_{N}$. In particular, the largest group that projects along $q_{N}$ is the normalizer of $\operatorname{Ker} q_{N}$ within Aut $X$.

Moreover, if a group $H \leq$ Aut $X$ projects, then the kernel of the induced group epimorphism $q_{N}^{*}: H \rightarrow \bar{H}$ is $H \cap \overline{K e r} q_{N}$. In particular, if $G \leq$ Aut $X_{N}$ lifts, then the kernel of the group epimorphism $\widetilde{G} \rightarrow G$ is $\operatorname{Ker} q_{N}$.

A quotient projection $q_{N}: X \rightarrow X_{N}$ is called normal if Aut $X$ projects along $q_{N}$, that is, if $\operatorname{Ker} q_{N} \triangleleft$ Aut $X$. In general, if $H$ projects along $q_{N}$ then a conjugate subgroup $\alpha \circ H \circ \alpha^{-1}$ 을 Springer 
projects along $q_{\left(\alpha \circ N \circ \alpha^{-1}\right)}$ (which might not be equal to $q_{N}$ ). From Proposition 3.1 it follows that, given a graph $X$ and $H \leq$ Aut $X$, one can find all quotient projections $q_{K}: X \rightarrow X_{K}$ along which $H$ projects-by letting $K$ run through all normal subgroups of all subgroups $H^{\prime} \leq$ Aut $X$ containing $H$. (The resulting projections are not necessarily pairwise distinct, nonequivalent or nonisomorphic; we do find all morphisms, but not necessarily all groups realising these morphisms.) Among these, all regular covering projections are obtained by selecting those subgroups $K$ which act semiregularly on $X$.

If an automorphism lifts along a quotient projection then it lifts along any quotient projection equivalent to it from above. Consequently, if a regular covering projection $\wp_{N}: X \rightarrow \bar{X}$ is reconstructed as $\wp_{\zeta}: \operatorname{Cov}(\bar{X} ; \zeta) \rightarrow \bar{X}$, then a necessary and sufficient condition for $G \leq$ Aut $\bar{X}$ to lift along $\wp_{N}$ can be expressed combinatorially in terms of voltages (in order for $G$ to lift along $\wp_{\zeta}$ ). Also, it is enough to require that each automorphism in some generating set of $G$ lifts; see Proposition 3.2 below. Equivalence and isomorphism of regular covering projections can also be studied along these lines; see Proposition 3.3 below.

Proposition 3.2. An automorphism $\alpha \in$ Aut $\bar{X}$ lifts along a regular covering projection $\wp_{\zeta}: \operatorname{Cov}(\bar{X} ; \zeta) \rightarrow \bar{X}$ if and only if each closed walk with trivial voltage is mapped to a walk with trivial voltage.

Proposition 3.3. Regular covering projections $\wp_{N_{i}}: \operatorname{Cov}\left(\bar{X} ; \zeta_{i}\right) \rightarrow \bar{X}$, for $i=1,2$, are isomorphic if and only if there exists a group isomorphism $\tau: N_{1} \rightarrow N_{2}$ and a graph automorphism $\alpha \in$ Aut $\bar{X}$ such that for each closed walk $W$ in $\bar{X}$ we have $\tau\left(\zeta_{1}(W)\right)=\zeta_{2}(\alpha(W))$. In particular, $\wp_{N_{1}}$ and $\wp_{N_{2}}$ are equivalent from above if and only if there exists an isomorphism $\tau: N_{1} \rightarrow N_{2}$ such that $\tau \circ \zeta_{1}=\zeta_{2}$.

Decomposition of quotient projections We say that a quotient projection $q_{N}: X \rightarrow X_{N}$ has a decomposition if it can be written as $q_{N}=q_{M} \circ q_{K}$ where $N, K \leq$ Aut $X$ and $M \leq$ Aut $X_{K}$. Such a decomposition is said to be nontrivial if neither of the projections $q_{K}$ or $q_{M}$ is an isomorphism.

Observe that the $K$-orbits are contained in the orbits of $N$ if and only if $\operatorname{Ker} q_{K} \leq \operatorname{Ker} q_{N}$. Consequently, a necessary condition for a nontrivial decomposition of $q_{N}$ to exist is that there be a subgroup $1 \neq K \leq \operatorname{Ker} q_{N}$ such that $\operatorname{Ker} q_{K}<\operatorname{Ker} q_{N}$. But the condition is not sufficient. Specifically, if the $K$-orbits are contained in the orbits of $N$, then one can define a graph morphism $f: X_{K} \rightarrow X_{N}$ such that $q_{N}=f \circ q_{K}$, however, this morphism does not necessarily arise from taking the quotient by the action of some group of automorphisms of $X_{K}$. Now suppose that $\operatorname{Ker} q_{K}<\operatorname{Ker} q_{N}$ is normalized by $N$, that is, let $N$ project along $q_{K}$. Then the projection $\bar{N} \cong N /\left(N \cap \operatorname{Ker} q_{K}\right)$ of $N$ along $q_{K}$ projects along $f$; in fact, the graph epimorphism $f$ is isomorphic to the projection $q_{\bar{N}}$. Such a decomposition is called strong. In particular, if $\operatorname{Ker} q_{K}$ is a nontrivial proper normal subgroup of $\operatorname{Ker} q_{N}$, then the decomposition is nontrivial and strong. But if a nontrivial decomposition is strong, then $\operatorname{Ker} q_{K}$ is not necessarily a normal subgroup of $\operatorname{Ker} q_{N}$, that is, $\operatorname{Ker} q_{N}$ might not project along $q_{K}$.

Regular covering projections are much better behaved with respect to the questions addressed above. We point out, firstly, that any decomposition $\wp_{N}=\wp_{M} \circ \wp_{K}$ (where all projections are required to be reqular covering projections) is necessarily strong. Secondly, since $N=\operatorname{Ker} \wp_{N}$ and $K=\operatorname{Ker} q_{K}$, we see that $K$ must be normal in $N$, and consequently, such a decomposition takes the form $\wp_{N}=\wp_{N / K} \circ \wp_{K}$. Also, a decomposition is trivial if and only if $K=1$ or $K=N$.

We close this section by introducing some additional terminology for later reference. If $\wp_{N}: X \rightarrow X_{N}$ is a regular covering projection and a group $G \leq X_{N}$ lifts along $\wp_{N}$ we say that 
$\wp_{N}$ is $G$-admissible. If $\wp_{N}$ is $G$-admissible but there exists no $G$-admissible regular covering projection $\wp_{N / K}$ such that $\wp_{N}=\wp_{N / K} \circ \wp_{K}$, then we say that $\wp_{N}$ is minimally $G$-admissible. Loosely speaking, $\wp_{N}$ is minimally $G$-admissible whenever $G$ lifts along $\wp_{N}$ but does not lift along any "smaller" regular covering projection which decomposes $\wp_{N}$. We also remark that if $\zeta$ denotes the voltage assignment realising $\wp_{N}$, then the voltage assignment which realises, up to equivalence, the covering projection $\wp_{N / K}$ (such that $\wp_{N}=\wp_{N / K} \circ \wp_{K}$ ), is given by $q \circ \zeta$, where $q: N \rightarrow N / K$ is the quotient projection. Further details on decomposing quotient and regular covering projections will be given in Section 6 .

\section{Families of cubic semisymmetric graphs}

In this section we give an overview of known families of cubic semisymmetric graphs. With the exception of families I, II and VII, they all arise as regular covers of certain cubic arc-transitive graphs of small orders.

Let $n$ be an integer such that there exists a unique cubic semisymmetric graph on $n$ vertices. We use the symbol Sn to denote this graph. As a natural extension of this notation we use the symbols Sna, Snb, ... in the case when nonisomorphic cubic semisymmetric graphs on $n$ vertices exist. For example, the smallest cubic semisymmetric graph, the so called Gray graph on 54 vertices, is denoted with symbol S54, while the three pairwise non-isomorphic cubic semisymmetric graphs on 336 vertices are denoted with symbols S336a, S336b and S336c. The proposed notation is analogous to that used in the Foster census [4] for cubic arc-transitive graphs on $n$ vertices, namely Fn (or Fna, Fnb, ...).

I. Let $n \geq 9$ be an integer divisible by 3 , let $A_{n}$ denote the alternating group acting on the set $\{1,2, \ldots, n\}$, and let $\alpha, \beta \in A_{n}$ be the following permutations:

$$
\begin{gathered}
\alpha=(1,2,3)(4,5,6)(7,8,9) \cdots(n-2, n-1, n), \\
\beta=(1,2,6)(4,5,9)(7,8,12) \cdots(n-5, n-4, n)(3)(n-2)(n-1) .
\end{gathered}
$$

Then the Cayley graph of $A_{n}$ relative to the generating set $\{\alpha, \beta\}$ is the line graph of a cubic semisymmetric graph of order $n ! / 3$ (see [33]). The vertex stabilisers are all isomorphic to $S_{3}$ and so the Goldschmidt type is $G_{1}^{1}$. The smallest graph in the family has 60480 vertices.

II. Two families of cubic semisymmetric graphs given in [28] can be defined using the amalgam completion construction described in Section 2. Namely, let $G=P G L(2, p)$ for $p$ a prime congruent to 11 or 13 modulo 24 and let $G=P S L(2, p)$ for a prime $p$ congruent to \pm 1 modulo 24 . Then $G$ contains a subgroup $P_{1}$ isomorphic to $D_{24}$, and a maximal subgroup $P_{2}$ isomorphic to $S_{4}$, which generate $G$ and intersect in a subgroup $B$ isomorphic to $D_{8}$. The pair of inclusion maps from $B$ into $P_{1}$ and $P_{2}$ is a finite primitive amalgam of index $(3,3)$ and type $G_{2}^{1}$ (see 2.2). Moreover, the pair of inclusion maps from $P_{i}$, for $i=1,2$, into $G$ is its faithful finite completion. As observed by Goldschmidt [20], the graph of this completion is a cubic $G$-semisymmetric graph. The semisymmetry of these graphs was proved in [28]. The smallest members, arising for primes $p=11,13,23$, are the three biprimitive graphs in our list: S110, S182 and S506. Note that other graphs in these two families are not biprimitive (since the group $P_{1} \cong D_{24}$ is not maximal in $G$ ). But as $P_{2} \cong S_{4}$ is maximal in $G$, the action of $G$ on one part of bipartition is primitive in all cases.

III. The next two families arise as covers of $K_{3,3}$ with a cyclic or elementary abelian voltage group $N$. In order to describe them via voltage assignments we choose the spanning 且 Springer 
tree carrying trivial voltages as shown in Figure 1. Furthermore, let $a, b, c, d \in N$ be the voltages of the remaining cotree arcs $(3,2),(3,4),(2,5)$ and $(4,5)$, respectively.

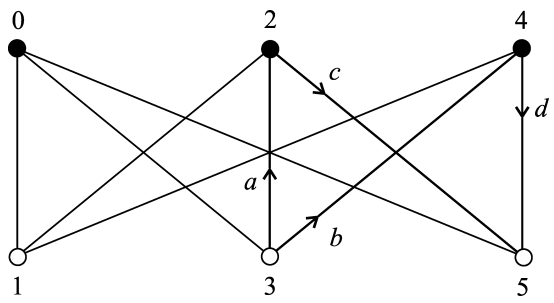

Fig. $1 K_{3,3}$ as base graph with spanning tree carrying trivial voltages

Let $n=3^{\varepsilon} p_{1}^{e_{1}} p_{2}^{e_{2}} \ldots p_{k}^{e_{k}}, k \geq 2$, where $\varepsilon \in\{0,1\}$ and $p_{i}, i=1, \ldots, k$, are distinct primes congruent to 1 modulo 3 , and let $N=\mathbb{Z}_{n}$. In this case there are two solutions $r, s \in \mathbb{Z}_{n}^{*}$ of the equation $x^{2}+x+1=0$ such that $r \neq s, s^{-1}$. Then, letting $a=1, b=-r, c=s$, and $d=-r s$, the corresponding regular $N$-cover of $K_{3,3}$ is semisymmetric with vertex stabilisers isomorphic to $\mathbb{Z}_{3}$ (see [30]). Hence the Goldschmidt type is $G_{1}$. The smallest graph in this family has 546 vertices, appearing in the list as S546a.

Along similar lines, let $p \equiv 1(\bmod 3)$ be a prime, let $N=\mathbb{Z}_{p}^{2}$, and let $r \in \mathbb{Z}_{p}$ be a primitive third root of unity. Then, by letting $a=(1,0), b=(0,1), c=(r, 0)$ and $d=(0, r)$, the corresponding regular $N$-cover of $K_{3,3}$ is semisymmetric (see [29]). We note that vertex stabilisers are isomorphic to $S_{3}$ for $p>7$, and so the Goldschmidt type is $G_{1}^{1}$ in such cases. On the other hand, $p=7$ gives rise to the smallest graph in the family on 294 vertices, appearing in the list as $\mathbf{S 2 9 4}$. Its Goldschmidt type is $G_{1}^{2}$.

IV. There are four infinite families of pairwise nonisomorphic regular elementary abelian covers of the Heawood graph (F14), where the maximal lifted subgroup acts semisymmetrically on the covering graph. The first two families arise, respectively, as $\mathbb{Z}_{p}^{3}$-covers and $\mathbb{Z}_{p}^{5}$-covers for $p \equiv 1,2$ or $4(\bmod 7)$, whereas the remaining two arise as $\mathbb{Z}_{p}^{4}$-covers when $p$ satisfies the additional condition $p \equiv 1(\bmod 3)$. Furthermore, there are three sporadic examples of regular $\mathbb{Z}_{7}^{6}$-covers of $\mathbf{F 1 4}$ satisying the above-mentioned property. The minimal members of the first two families are the graphs S112 and S448 in the list. Their Goldschmidt type is $G_{1}$. We remark that for the remaining graphs the semisymmetry of the full automorphism group has not yet been verified.

In order to describe these constructions, we let the arcs of the Hamilton path $5,0^{\prime}, 6,1^{\prime}, \ldots, 3,5^{\prime}, 4,6^{\prime}$ of $\mathbf{F 1 4}$ carry the trivial voltage, and we let $a_{7}, a_{0}, a_{1}, \ldots, a_{6}$ denote the voltages of the arcs $\left(5,6^{\prime}\right),\left(0,4^{\prime}\right),\left(1,5^{\prime}\right),\left(2,6^{\prime}\right),\left(3,0^{\prime}\right),\left(4,1^{\prime}\right)$, $\left(5,2^{\prime}\right),\left(6,3^{\prime}\right)$, respectively (see Figure 2$)$. To obtain the three $\mathbb{Z}_{7}^{6}$-covers, let $a_{7}=(1,0,0,0,0,0), a_{0}=(0,1,0,0,0,0), \ldots, a_{4}=(0,0,0,0,0,1)$ be the six standard basis vectors of $\mathbb{Z}_{7}^{6}$, and let $a_{5}=(x, 1,2,3,4,5)$ and $a_{6}=(y, 5,4,3,2,1)$, where $(x, y)=$ $(6,6),(3,2)$ or $(0,5)$, respectively. The corresponding three covering graphs on 14 . $7^{6}=1647086$ vertices arise from pairwise non-isomorphic covering projections.

For the remaining infinite families, the prime $p$ satisfies the condition $p \equiv 1,2$ or $4(\bmod 7)$. In this case there exists an element $s \in \mathbb{Z}_{p}$ satisfying $s^{2}+s+2=0$. The infinite family of $\mathbb{Z}_{p}^{3}$-covers is obtained by letting $a_{7}=(0,0,0), a_{0}=(1,0,0)$, $a_{1}=(0,1,0), a_{2}=(0,0,1), a_{3}=(1, s+1, s), a_{4}=(s,-1,-1), a_{5}=(-1,-1,-s-1)$, and $a_{6}=(-s-1,-s, 1)$. Similarly, the infinite family of $\mathbb{Z}_{p}^{5}$-covers is obtained by letting $a_{7}=(1,0,0,0,0), a_{0}=(0,1,0,0,0), a_{1}=(0,0,1,0,0), a_{2}=(0,0,0,1,0)$, $a_{3}=(0,0,0,0,1)$ be the standard basis vectors of $\mathbb{Z}_{p}^{5}$, and by setting $a_{4}=(s,-1,-s, 1, s+$ $1), a_{5}=(s,-s-1,1,1, s)$, and $a_{6}=(-1,-s, 1, s+1,-1)$. Finally, let $p$ satisfy the addi- 


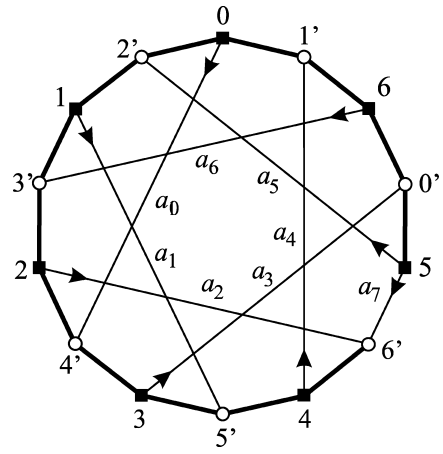

Fig. 2 The Heawood graph F14 as base graph with bold spanning tree carrying trivial voltages

tional condition $p \equiv 1(\bmod 3)$ and let $r_{1}, r_{2}$ be the two primitive cube roots of unity in $\mathbb{Z}_{p}$. Then the two infinite families of $\mathbb{Z}_{p}^{4}$-covers are obtained for $i=1,2$ by letting $a_{7}=\left(r_{i}+\right.$ $3,0,0,0), a_{0}=(1,1,0,0), a_{1}=(1,0,1,0), a_{2}=(1,0,0,1), a_{3}=\left(-r_{i}-2,1, s+1, s\right)$, $a_{4}=\left(-r_{i}-2, s,-1,-1\right), a_{5}=(1,-1,-1,-s-1)$ and $a_{6}=(1,-s-1,-s,-1)$.

V. Apart from the graph $\mathbf{S 1 4 4}$, with Goldschmidt type $G_{1}^{1}$, which is a regular $\mathbb{Z}_{3}^{2}$-cover of the Moebius-Kantor graph (F16), there is an infinite family of regular elementary abelian covers of F16 where the maximal lifted subgroup acts semisymmetrically on the covering graphs. These graphs arise as $\mathbb{Z}_{p}^{2}$-covers, where $p \equiv 1(\bmod 4)$ is a prime. The smallest graph in this family is $\mathbf{S 4 0 0}$. Its Goldschmidt type is $G_{1}$. As in IV above, the semisymmetry of the full automorphism group for the remaining graphs in the family has not been verified.

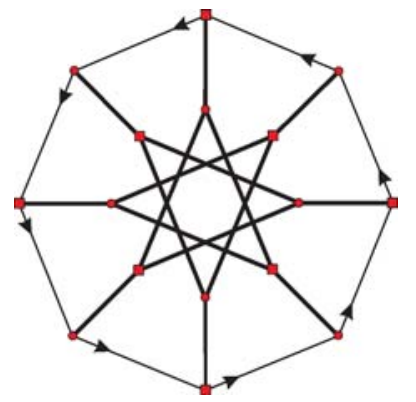

Fig. 3 The Moebius-Kantor graph F16 as base graph with bold edges carrying trivial voltages

In order to describe these constructions we observe that F16 is isomorphic to the generalised Petersen graph $G P(8,3)$, thus allowing us to identify its vertex set with $\mathbb{Z}_{8} \times \mathbb{Z}_{2}$ and its edge set with

$$
E=\left\{\left\{(i, 0),(i+1,0\} \mid i \in \mathbb{Z}_{8}\right\} \cup\left\{\{(i, 1),(i+3,1)\} \mid i \in \mathbb{Z}_{8}\right\} \cup\left\{\{(i, 0),(i, 1)\} \mid i \in \mathbb{Z}_{8}\right\} .\right.
$$

Define the voltages on the arcs of the inner cycle and on the spokes to be trivial. As for the arcs of the oriented outer cycle, we define these to be, respectively, $(1,0),(0,1),(0,1)$, $(-1,0),(-1,0),(0,-1),(0,-1),(1,0)$, in the case $p=3$. Similarly, for the infinite family of regular $\mathbb{Z}_{p}^{2}$-covers, $p \equiv 1(\bmod 4)$, we let the above voltages be, respectively, $(1,0),(0,1)$, $(r, r-1),(0,-r),(-1,0),(0,-1),(-r,-r-1),(0, r)$, where $r^{2}=-1$ (see Figure 3.) 
VI. There exists an infinite family of regular elementary abelian covers of the Pappus graph (F18) where the maximal lifted subgroup acts semisymmetrically on the covering graph. The graphs in this family arise as $\mathbb{Z}_{p}^{2}$-covers, where $p \equiv 1(\bmod 3)$ is a prime. With the exception of the smallest graph $(p=7)$, which has 882 vertices and is indeed semisymmetric, the semisymmetry of the full automorphism group for the remaining graphs in the family has not been verified.

To describe this construction we identify the vertex set of $\mathbf{F 1 8}$ with $\{1,2, \ldots, 18\}$ and its edge set with the union of the sets $\{\{i, i+1\} \mid i \in\{1,2, \ldots, 18\}\},\{\{i, i+5\} \mid i \in\{1,7,13\}\}$, $\{\{i, i+7\} \mid i \in\{2,8,14\}\}$, and $\{\{i, i+7\} \mid i \in\{4,10,16\}\}$, where the arithmetic is performed modulo 18 . We let the arcs of the Hamilton path $(1,2, \ldots, 18)$ carry trivial voltages (see Figure 4), and we let the arcs $\{(i, i+5) \mid i \in\{1,7,13\}\},\{(i, i+7) \mid i \in\{2,8,14\}\},\{(i, i+$ 7) $\mid i \in\{4,10,16\}\}$, and $(18,1)$, respectively, have the following voltages: $(1,0),(1,0),(1,0)$, $(0,1),(-r+1, r),(-1,-r-1),(-r, r+1),(2,-1),(-2,-r)$, and $(-r-3,0)$, where $r$ is an element of order 3 in $\mathbb{Z}_{p}^{*}$.

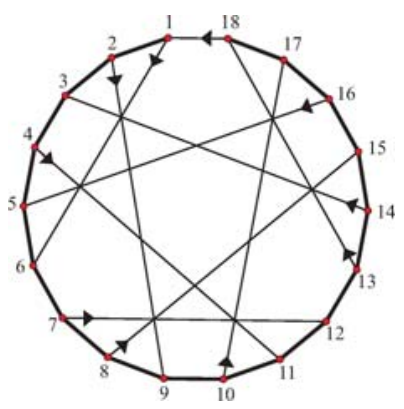

Fig. 4 The Pappus graph F18 as base graph with bold spanning tree carrying trivial voltages

VII. There are eight graphs in the list with the tripod $K_{1,3}$ as a normal quotient: S120, S330, S336b, S336c, S504, S546b, S660 and S720b. These graphs may be obtained using a general approach described in the next section.

\section{Semisymmetric actions arising from $K_{1,3}$}

In this section we prove a theorem which enables us to construct several infinite families of semisymmetric cubic graphs. It deals with the situation where a semisymmetric group of automorphisms contains a normal simple subgroup acting transitively on one part of the bipartition and intransitively on the other. It came to our notice while preparing the final version of this paper that a similar problem was considered by Giudici, Li and Praeger [17].

First we prove the following, which relies heavily on the analysis and classification of finite arc-transitive cubic graphs by Tutte [37] and Djoković and Miller [11], and on an indepth analysis of their automorphism groups in terms of generators and relations by Conder and Lorimer [5]. In particular, it is known that an arc-transitive group of automorphisms of a finite cubic graph $X$ acts regularly on the set of $k$-arcs of $X$ for some $k \in\{1,2,3,4,5\}$, and its action falls into one of seven possible classes, denoted in [5] by $G_{1}, G_{2}^{1}, G_{2}^{2}, G_{3}, G_{4}^{1}$, $G_{4}^{2}$ and $G_{5}$. We shall call these classes Djoković-Miller classes, to distinguish them from the Goldschmidt types of semisymmetric group actions. 
Proposition 5.1. Let $X$ be a connected cubic bipartite graph admitting an arc-transitive action of a group $G \leq$ Aut $X$, and a semisymmetric action of a subgroup $H \leq G$. Also let $T$ be the maximal subgroup of $G$ preserving both parts of the bipartition of $X$. If $H$ is selfnormalising in $G$, that is, if $N_{G}(H)=H$, then $H$ is of Goldschmidt type $G_{1}$, and $G$ belongs to the Djoković-Miller class $G_{4}^{2}$, and the permutation representation induced by $T$ (by right multiplication) on right cosets of $H$ is isomorphic to the natural action of $P S L(2,7)$ on the projective line $P G(1,7)$.

Proof: The proof is divided into several cases, dealing in turn with the seven possible Djoković-Miller classes to which $G$ might belong. It follows from [5] that in each of these cases, the group $G$ is generated by an element $h$ (of order 3 ) fixing a vertex $v$, an element $a$ (of order 2 or 4) reversing an arc $(v, w)$ incident with $v$, and up to four involutions (denoted by $p, q, r$ and $s)$ which generate the stabiliser of the arc $(v, w)$. Also the vertex-stabilisers $G_{v}$ and $G_{w}$ are generated by the stabiliser of the $\operatorname{arc}(v, w)$ and elements $h$ and $h^{a}=a^{-1} h a$, respectively. Moreover, since the order of $G_{v}$ divides $3 \cdot 2^{4}$ (by Tutte's theorem), all cyclic subgroups of order 3 fixing the vertex $v$ are conjugate in $G_{v}$.

We consider possibilities for $H$ in terms of two elements $x$ and $y$, each of order 3 in $H$, and stabilizing $v$ and $w$ respectively. Without loss of generality we may suppose that $x$ is the element $h$ used as a generator for $G$. Furthermore, since $v^{a}=w$ we know that $a y a^{-1}$ fixes $v$, and therefore also $a y a^{-1}$ is an element of order 3 in $G_{v}$.

Now if $G$ belongs to one of the Djoković-Miller classes $G_{1}, G_{2}^{1}$ or $G_{2}^{2}$, then $\left|T_{v}\right|=\left|G_{v}\right|=3$ or 6, so the index $|T: H|=\left|T_{v}: H_{v}\right|$ is either 1 or 2 , and hence $H$ is normal in $T$, contradicting the assumption $N_{G}(H)=H$.

If $G$ belongs to the Djoković-Miller class $G_{3}$, then $G_{v} \cong S_{3} \times C_{2}$ is generated by $h$ and two involutions $p$ and $q$ such that $p q=q p, p h p=h, q h q=h^{-1}$ and $a p=q a$, and $a$ itself is an involution. Clearly, $G_{v}$ has only two elements of order 3, so $a y a^{-1}=h$ or $h^{-1}$. In both cases, the group $\left\langle h, h^{a}\right\rangle=\langle x, y\rangle \leq H$ has index dividing 4 in $T$ with transversal a subset of $\{1, p, q, p q\}$. Since $N_{G}(H)=H$, the index of $H$ in $T$ is at least 4. It follows that $H=\left\langle h, h^{a}\right\rangle$, but this subgroup is normalised by $a$, a contradiction.

If $G$ belongs to the Djoković-Miller class $G_{4}^{1}$ or to the class $G_{4}^{2}$, then $G_{v} \cong S_{4}$ is generated by $h$ and three involutions $p, q$ and $r$ such that $p q=q p, p r=r p,(q r)^{2}=p, h^{-1} p h=q$, $h^{-1} q h=p q, r h r=h^{-1}, a p=p a$ and $a q=r a$, with $a^{2}=1$ in the case of Djoković-Miller class $G_{4}^{1}$, while $a^{2}=p$ in the case of Djoković-Miller class $G_{4}^{2}$. This time we find that $G_{v}$ has eight elements of order 3, and so $a y a^{-1}=h^{ \pm 1}$ or $p h^{ \pm 1} p$ or $q h^{ \pm 1} q$ or $q p h^{ \pm 1} p q$. Thus $H$ contains one of the four subgroups $L_{1}=\left\langle h, a^{-1} h a\right\rangle, L_{2}=\left\langle h, a^{-1} p h p a\right\rangle, L_{3}=\left\langle h, a^{-1} q h q a\right\rangle$ or $L_{4}=\left\langle h, a^{-1} q\right.$ phpqa $\rangle$. These subgroups all have index dividing 16 in $G$, and index dividing 8 in $T=\left\langle h, a^{-1} h a, p, q, r\right\rangle$, with transversal in $T$ being a subset of the dihedral subgroup $A=\langle p, q, r\rangle$ of order 8. Since $h^{-1} p h=q, h^{-1} q h=p q, h^{-1} p q h=p$, it follows that if $H$ contains one of the elements $p, q$ and $p q$, then it contains all of them, and has therefore index at most 2 in $T$, a contradiction. Observe also that $r^{-1} L_{1} r=L_{3}$ while $r^{-1} L_{2} r=L_{4}$.

In the case of $G_{4}^{1}$, the subgroup $L_{1}$ is normalised by $a$, while $L_{2}, L_{3}$ and $L_{4}$ are normalised by ap, aqr and arq respectively. Hence the index of $H$ in $T$ has to be 4, and $H$ must be of the form $\left\langle L_{i}, u\right\rangle$ where $u$ is an element of $A \backslash\langle p, q\rangle=\{r, p r, q r, p q r\}$ normalizing $L_{i}$. It is easy to check, however, that this is impossible for all four subgroups $L_{i}$; for example, $p r h r p=p h^{-1} p=p q h^{-1}$ but $p q \notin H$, and although $r h r=h^{-1}$ we have also $r\left(a^{-1} h a\right) r=r a^{-1} h q a=r a^{-1} p h a=r p a^{-1} h a=p\left(a^{-1} h a\right)$ but $p \notin H$.

In the case of $G_{4}^{2}$, observe that $a^{-1} p L_{1} p a=\left\langle a^{-1}\right.$ phpa, $a^{-1}$ pahapa $\rangle=\left\langle a^{-1} p h p a\right.$, $h\rangle=L_{2}$ since $a p a=a^{4}=p^{2}=1$, and hence that all four subgroups $L_{1}, L_{2}, L_{3}$ and $L_{4}$ are conjugate to $L=L_{1}=\left\langle h, a^{-1} h a\right\rangle$ in $G$. We can therefore assume without loss of generality 
that $L \leq H$. Now consider the action of the group $T$ on the right cosets of its subgroup $L$. The permutations induced by its generators $h, a^{-1} h a, p, q$ and $r$ are as follows:

- $h$ induces the permutation $(L)(L p, L q, L p q)(L p r, L p q r, L q r)(L r)$;

- $a^{-1} h a$ induces the permutation $(L)(L p, L r, L p r)(L q)(L p q, L q r, L p q r)$;

- $p$ induces the permutation $(L, L p)(L q, L p q)(L r, L p r)(L q r, L p q r)$;

- $q$ induces the permutation $(L, L q)(L p, L p q)(L r, L p q r)(L p r, L q r)$; and

- $r$ induces the permutation $(L, L r)(L p, L p r)(L q, L q r)(L p q, L p q r)$.

These permutations generate a group of order 168 isomorphic to $\operatorname{PSL}(2,7)$ in its natural representation on the projective line over $\mathrm{GF}(7)$, as may be seen from the correspondence which takes ( $L, L p, L q r, L p q, L q, L r, L p r, L p q r)$ to $(\infty, 0,1,2,3,4,5,6)$, and the permutations induced by the generators $h, a^{-1} h a, p, q$ and $r$ of $T$ to the transformations $z \mapsto 2 z+1$, $z \mapsto 2 z-1, z \mapsto 3 / z, z \mapsto(z-3) /(z-1)$ and $z \mapsto(-z-3) /(z+1)$ respectively. In particular, as this permutation representation is primitive, we find that $L \leq H$ is a maximal subgroup of $T$, implying $L=H$, as required.

Finally, if $G$ belongs to Djoković-Miller class $G_{5}$, then $G_{v} \cong S_{4} \times C_{2}$ is generated by $h$ and four involutions $p, q, r$ and $s$ such that $p q=q p, p r=r p, p s=s p, q r=r q, q s=s q$, $(r s)^{2}=p q, h^{-1} p h=p, h^{-1} q h=r, h^{-1} r h=p q r, s h s=h^{-1}, a p=q a$ and $a r=s a$, and $a$ itself is an involution. Again we find that $G_{v}$ has eight elements of order 3, so $a y a^{-1}=h^{ \pm 1}$ or $q h^{ \pm 1} q$ or $r h^{ \pm 1} r$ or $r q h^{ \pm 1} q r$, and hence $H$ contains at least one of $M_{1}=\left\langle h, a^{-1} h a\right\rangle$, $M_{2}=\left\langle h, a^{-1} q h q a\right\rangle, M_{3}=\left\langle h, a^{-1} r h r a\right\rangle$ and $M_{4}=\left\langle h, a^{-1} r q h q r a\right\rangle$. These subgroups all have index dividing 32 in $G$, and index dividing 16 in $T$, with transversal in $T$ being a subset of the subgroup $A=\langle p, q, r, s\rangle \cong D_{4} \times C_{2}$. Also $p^{-1} M_{1} p=M_{2}$ and $s^{-1} M_{1} s=M_{3}$ and $(p s)^{-1} M_{1} p s=M_{4}$, so that all four subgroups are conjugate in $T$, and hence we may suppose that $H$ contains $M=M_{1}=\left\langle h, a^{-1} h a\right\rangle$. Moreover, as $M$ is normalised by $a$, we know $H \neq M$ and therefore $H$ must contain at least one element of $A=\langle p, q, r, s\rangle$. But if $H$ contains $p$, then $H$ contains $\left(a^{-1} h a\right)^{-1} p\left(a^{-1} h a\right)=s$ and $\left(a^{-1} h a\right) p\left(a^{-1} h a\right)^{-1}=p q s$, and therefore contains also $p(p q s) s=q$ and $h^{-1} q h=r$, and so contains all of $T$, contradiction. Hence $p \notin H$. A similar argument shows that $H$ contains none of $\{p, q, r, s, p q r, p q s\}$. Furthermore, this shows $p r s \notin H$, for otherwise $H$ would contain $h p r s h=p h r h^{-1} s=p q s$, and similarly $q r s \notin H$. On the other hand, if $H$ contains $p q$, then $H$ contains also $h^{-1} p q h=p r$ and $\left(a^{-1} h a\right) p q\left(a^{-1} h a\right)^{-1}=p s$, and therefore contains the subgroup $\left\langle h, a^{-1} h a, p q, p r, p s\right\rangle$, which has index 2 in $T$, contradiction. Hence $p q \notin H$, and the same sort of argument shows that $H$ contains none of $\{p q, p r, p s, q r, q s\}$. In particular, $r s \notin H$ (for otherwise $\left.p q=(r s)^{2} \in H\right)$, and similarly pqrs $=s r \notin H$.

This completes the proof.

Theorem 5.2. Let $X$ be a connected cubic bipartite edge-transitive graph with automorphism group $G$, and let $S$ be a simple group other than $\mathbb{Z}_{3}$ such that $S$ is normal in some semisymmetric group $H \leq G$, and such that the quotient $X_{S}$ is isomorphic to $K_{1,3}$. Then the following hold:

(i) $N_{G}(H) \leq N_{G}(S)$;

(ii) $N_{G}(S)$ acts semisymmetrically on $X$;

(iii) $N_{G}(S)$ is a self-normalising subgroup of $G$, that is, $N_{G}\left(N_{G}(S)\right)=N_{G}(S)$;

(iv) $X$ is semisymmetric.

Proof: Observe first that $H$ acts transitively on both parts of the bipartition of $X$, and that $S$ acts transitively on one part and intransitively (with three orbits of equal length) on the other 
part. This implies that

$$
|H: S|=\left|H_{u}: S_{u}\right|=3\left|H_{v}: S_{v}\right|
$$

where $u$ is a vertex of the part on which $S$ acts transitively and $v$ is a vertex of the other part. In particular, by Goldshmidt's theorem [20], the index $|H: S|$ divides $3 \cdot 2^{s-1}$ for some $s \leq 9$. (Moreover, if $X$ were symmetric, then we would have $s \leq 5$ by the earlier theorem of Tutte [37]). Note also that since $S$ is transitive on one part of the bipartition, and the cardinality of both parts is divisible by 3 , the cardinality of $S$ is divisible by 3 , and $|S|>3$.

Let us now prove part (i). Assume the contrary. Then there exists an element $g \in G$ which normalises $H$ but does not normalise $S$. It follows that the conjugate $S^{g}$ is a normal subgroup of $H^{g}=H$, so also $S \cap S^{g}$ is a normal subgroup of $S$, and by simplicity of $S$, we find $S \cap S^{g}=1$. This implies that the normal subgroup $S S^{g}$ of $H$ is isomorphic to the direct product $S \times S$, of order $|S|^{2}$. In particular, the index $|H: S|$ is divisible by $|S|$, and so by (*), $|S|$ divides $3 \cdot 2^{s-1}$, and hence $S \cong \mathbb{Z}_{2}$ or $\mathbb{Z}_{3}$, contradicting the fact that $|S|>3$. Thus $N_{G}(H) \leq N_{G}(S)$.

To prove parts (ii) and (iii), observe that $S$ cannot be normal in any vertex-transitive subgroup of $G$, since its actions on the two parts of the bipartition are not equivalent. In particular, $N_{G}(S)$ is edge- but not vertex-transitive. But now applying part (i) to $N_{G}(S)$ in place of $H$, we find $N_{G}\left(N_{G}(S)\right) \leq N_{G}(S)$, and thus $N_{G}(S)$ is a self-normalising semisymmetric subgroup of $G$.

Finally, let us prove that the graph $X$ is not vertex-transitive. Assume the contrary. As a consequence of part (ii) we can assume without loss of generality that $N_{G}(H)=H$. Hence by Proposition 5.1, the group $H$ has a homomorphic image isomorphic to a point-stabiliser in the natural action of $P S L(2,7)$ on the projective line $P G(1,7)$. This is a group of order 21, and so $H$ must have both the cyclic groups $C_{3}$ and $C_{7}$ among its composition factors. On the other hand, we know that $H$ contains a non-abelian simple normal subgroup $S$ of index dividing $3 \cdot 2^{s-1}$ for some $1 \leq s \leq 5$, and so $C_{7}$ cannot be a composition factor of $H$. This contradiction completes the proof.

In what follows we construct some infinite families of connected cubic graphs, the semisymmetry of which follows from Theorem 5.2.

Construction 5.3. Let $G$ be the direct product $C \times S$, where $C$ is a cyclic group generated by an element $c$ of order 3 , and $S$ is a non-abelian simple group that can be generated by two elements $a$ and $b$ of order 3. Define $P_{1}=\langle c a\rangle$ and $P_{2}=\langle b\rangle$, and observe that the pair of inclusions $\left(P_{1} \rightarrow G, P_{2} \rightarrow G\right)$ is a faithful finite completion of the amalgam $\left(1 \rightarrow P_{1}, 1 \rightarrow\right.$ $P_{2}$ ). Now take $X$ to be the graph of this completion, with vertex-set the union $G / P_{1} \cup G / P_{2}$ of right cosets of $P_{1}$ and $P_{2}$ in $G$, and with two cosets $P_{1} x$ and $P_{2} y$ adjacent in $X$ if and only if $P_{1} x \cap P_{2} y \neq \emptyset$. Observe also that $G$ acts on $X$ by right multiplication of cosets, and that this action is semisymmetric (of Goldschmidt type $G_{1}$ ). Moreover, the subgroup $S$ acts transitively on the coset space $\left(G: P_{1}\right)$ but has three orbits on $\left(G: P_{2}\right)$. The corresponding quotient graph $X_{S}$ is therefore isomorphic to $K_{1,3}$, and so by Theorem 5.2, the graph $X$ is semisymmetric.

Note that graphs S120, S336b, S336c and S720b in our list can be obtained in this way. To illustrate its generality, we remark that many simple groups can be generated by two elements of order 3, and can thus be used in the above construction. Among these are all alternating groups $A_{n}$ for $n \geq 3$, all $P S L_{2}(p)$ and $P S L_{3}(p)$ for prime $p$, all $P S L_{n}(q)$ and $P S U_{n}(q)$ for large $n$ and all prime powers $q$, many other families of simple groups of Lie type, and most 은 Springer 
of the sporadic simple groups, including the Monster. (Note that most of these groups can be generated by two elements $x$ and $y$ of orders 2 and 3, and then simplicity implies that these groups can also be generated by $y$ and $y^{x}$.)

The next construction is a generalisation of 5.3.

Construction 5.4. Let $\left(\phi_{1}: B \rightarrow P_{1}, \phi_{2}: B \rightarrow P_{2}\right)$ be a finite primitive amalgam of index $(3,3)$, such that for $i=1$ or 2 (say $i=2$ ), the image $\operatorname{Im} \phi_{i}$ is normal in $P_{i}$. By Theorem 2.2 there are exactly four isomorphism classes of such amalgams, namely $G_{1}, G_{1}^{2}, G_{2}$ and $G_{2}^{3}$. Further, let $\left(\psi_{1}: P_{1} \rightarrow S, \psi_{2}: P_{2} \rightarrow S\right)$ be a faithful finite completion of the above amalgam, where $S$ is a simple group. Without loss of generality we may assume that homomorphisms $\phi_{i}$ and $\psi_{i}$ are inclusions (for $i=1$ and 2), so that $P_{1}$ and $P_{2}$ are subgroups generating $S$, and $B=P_{1} \cap P_{2}$. Note that by Proposition 2.1 this is equivalent to the following situation: $S$ is a simple group acting semisymmetrically on a connected bipartite cubic graph, such that the intersection $B$ of the stabilisers $P_{1}$ and $P_{2}$ of two adjacent vertices is normal in $P_{2}$.

Now let $C$ be a cyclic group of order 3 generated by an element $z$, and let $G$ be the direct product $C \times S$. Since $B$ is a normal 2-subgroup of index 3 in $P_{2}$, the group $P_{2}$ is isomorphic to the semidirect product $B \rtimes\langle y\rangle$, where $y^{3}=1$, and there exists a natural epimorphism $\pi: P_{2} \rightarrow\langle y\rangle$ with kernel $B$. We can now define group homomorphisms

$$
f_{1}: P_{1} \rightarrow C \times S \text { by } f_{1}(g)=(1, g), \quad \text { and } f_{2}: P_{2} \rightarrow C \times S \text { by } f_{2}(g)=(\pi(g), g) \text {. }
$$

These homomorphisms are clearly injective, and the simplicity of $S$ implies that their images generate $C \times S$. Indeed, let $H$ be the group generated by $\operatorname{Im} f_{1}$ and $\operatorname{Im} f_{2}$. Since $P_{1}$ and $P_{2}$ generate $S$, the projection of $H$ onto the second factor of $C \times S$ is $S$ itself, and so $H$ has index 1 or 3 in $C \times S$. In the latter case, $H$ would be isomorphic to $S$, and therefore simple. Also $S \cap H$ would be a normal subgroup of $H$ of index 1 or 3 , and since $H$ is simple and $|H|=|S|>3$, the index cannot be 3, so $S \cap H=H$. This is impossible, however, because $f_{2}(x)=(z, x) \notin S$. Thus $H=G=C \times S$.

The above implies that the pair of homomorphisms $\left(f_{1}, f_{2}\right)$ gives a finite faithful completion of the amalgam $\left(\phi_{1}, \phi_{2}\right)$. Let $X$ be the graph of this completion, with vertex-set $\left(G: \operatorname{Im} f_{1}\right) \cup\left(G: \operatorname{Im} f_{2}\right)$. By Proposition 2.1, the group $G=C \times S$ acts semisymmetrically on $X$. Observe also that its subgroup $S$ acts transitively on $\left(G: \operatorname{Im} f_{2}\right)$, but has three orbits on $\left(G: \operatorname{Im} f_{1}\right)$. The quotient graph $X_{S}$ is therefore isomorphic to $K_{1,3}$, and by Theorem 5.3, $X$ is semisymmetric.

This construction may be used to obtain the graphs S330, S546b and $\mathbf{S 6 6 0}$ in our list. (Also Construction 5.3 can be obtained as a special case of this one, by choosing the amalgam $\left(\phi_{1}, \phi_{2}\right)$ to be of type $G_{1}$.)

The following proposition introduces a third construction, for producing a connected bipartite cubic graph that admits a semisymmetric action of a semidirect product of a simple group by a cyclic group of order 3 .

Proposition 5.5. Let $S$ be a simple group which has an automorphism $\omega$ of order 3 , and let $B$ and $P$ be proper subgroups of $S$ such that:

(i) $B$ is a subgroup of $P$ of index 3 , and $B$ is either trivial or not normal in $P$;

(ii) $B^{\omega}=B$;

(iii) The subgroup generated by $P, P^{\omega}$ and $P^{\omega^{2}}$ is $S$. 
Further, let $G$ be the semi-direct product $S \rtimes\langle\omega\rangle$, and define $P_{1}=P$ (viewed as a subgroup of $G$ ) and $P_{2}=\langle B, \omega\rangle$, the subgroup of $G$ generated by $B$ and $\omega$ (isomorphic to the semi-direct product $B \rtimes\langle\omega\rangle)$. Then:

(a) $B=P_{1} \cap P_{2}$, and the pair of inclusions $\left(\phi_{1}: B \rightarrow P_{1}, \phi_{2}: B \rightarrow P_{2}\right)$ is a primitive amalgam of index $(3,3)$;

(b) the pair of inclusions $\left(\psi_{1}: P_{1} \rightarrow G, \psi_{2}: P_{2} \rightarrow G\right)$ is a faithful finite completion of the amalgam $\left(\phi_{1}, \phi_{2}\right)$;

(c) the graph $X$ of this completion admits a semisymmetric action of $G$, such that the quotient graph $X_{S}$ is isomorphic to $K_{1,3}$;

(d) $X$ is a connected semisymmetric cubic graph.

Proof: Everything in part (a) is obvious except for the fact that the amalgam $\left(\phi_{1}, \phi_{2}\right)$ is primitive. So let us assume that $\left(\phi_{1}, \phi_{2}\right)$ is not primitive. Then there exists a non-trivial subgroup $K$ of $B$ such that either:

- $K$ is normal in $P_{1}$ and the normaliser $N_{P_{2}}(K)$ properly contains $B$, or

- $K$ is normal in $P_{2}$ and the normaliser $N_{P_{1}}(K)$ properly contains $B$.

In the former case $N_{P_{2}}(K)=P_{2}$, since $B$ is maximal in $P_{2}$, and so $\omega \in P_{2}$ normalises $K$ and therefore $K$ is normal in $\left\langle P_{1}, P_{1}^{\omega}, P_{1}^{\omega^{2}}\right\rangle=S$, but that is impossible since $S$ is simple. In the latter case $N_{P_{1}}(K)=P_{1}$ since $B$ is maximal in $P_{1}$, while $\omega$ normalises $K$ because $\omega \in P_{2}$, and again $K$ is normal in $\left\langle P_{1}, P_{1}^{\omega}, P_{1}^{\omega^{2}}\right\rangle=S$, which is impossible because $S$ is simple.

To prove part (b) we need only show that $P_{1}$ and $P_{2}$ generate $G$. This follows directly from the fact that $\left\langle P, P^{\omega}, P^{\omega^{2}}\right\rangle=S$ implies $G=\langle S, \omega\rangle=\langle P, \omega\rangle=\left\langle P_{1}, P_{2}\right\rangle$.

For part (c), recall that the two parts of the bipartition of $X$ consist of right cosets of $P_{1}$ and $P_{2}$ in $G$, respectively, and that $G$ acts as a group of automorphisms of $X$ by right multiplication. Clearly $S \leq G$ acts transitively on the cosets of $P_{2}=\langle\omega\rangle B$ in $G=\langle\omega\rangle S$, in essentially the same way as $S$ acts on the cosets of $B$. On the other hand, $S$ acts intransitively on the cosets of $P_{1}=P$ in $G$, with three orbits corresponding to the cosets $S, \omega S$ and $\omega^{2} S$. The quotient graph $X_{S}$ is thus indeed isomorphic to $K_{1,3}$.

Finally, part (d) follows immediately from Theorem 5.2.

Example 5.6. Let $F=G F\left(2^{3}\right)$ be the finite field with 8 elements, let $\lambda$ be the generator of the multiplicative group $F^{*}$ satisfying $\lambda^{3}+\lambda+1=0$, and let

$$
b=\left[\begin{array}{ll}
0 & 1 \\
1 & 0
\end{array}\right] \quad \text { and } \quad a=\left[\begin{array}{cc}
\lambda & \lambda^{-1} \\
\lambda^{-1} & \lambda^{3}
\end{array}\right]
$$

be two elements of $S=S L(2, F) \cong P S L(2,8)$. Also let $B=\langle b\rangle$ and $P=\langle B, a\rangle$. Note that $P$ is a subgroup of $S$ isomorphic to $S_{3}$. Next let $\omega$ be the automorphism of $F$ mapping $\lambda$ to $\lambda^{2}$, which has order 3 since $\lambda^{\omega^{3}}=\lambda^{8}=\lambda$. It is easy to check that $\left\langle a, a^{\omega}, a^{\omega^{2}}\right\rangle=S$, so all conditions of Proposition 5.5 are satisfied. The resulting graph $X$ is a connected semisymmetric cubic graph on 504 vertices, isomorphic to the graph $\mathbf{S 5 0 4}$ in our list.

Example 5.7. Let $S, a$ and $\omega$ be as in the previous example, but let $B$ be the trivial subgroup of $S$, and $P=\langle a\rangle$. Then the conditions of Proposition 5.5 are again satisfied, and the resulting graph is a connected semisymmetric cubic graph on 1008 vertices. 


\section{The diagrams-explaining the list}

In this section we explain some of the structure and number of aspects of the list of semisymmetric graphs provided in Section 7. To do this, we need to discuss relationships between members of a class of graphs, as follows.

Irreducible morphisms and direct objects Let $\mathcal{C}$ be a class of graphs and graph morphisms (not necessarily a subcategory of graphs). A graph in $\mathcal{C}$ is called basic if it has no morphism in $\mathcal{C}$ to another graph in $\mathcal{C}$. A morphism $X \rightarrow Y$ in $\mathcal{C}$ is irreducible if it cannot be written as a composition of two morphisms (neither being an isomorphism) in $\mathcal{C}$. A graph $X$ is a direct object over a graph $Y$ if the $\operatorname{set} \operatorname{Hom}_{\mathcal{C}}(X, Y) \neq \emptyset$ consists of irreducible morphisms only (which are then called direct morphims). For example, in the context of all graphs and regular covering projections, $\wp: X \rightarrow Y$ is irreducible if and only if CT( $\wp)$ is a simple group; in addition, if CT( $\wp)$ is abelian then $X$ is a direct object over $Y$, but might fail to be direct if $\mathrm{CT}(\wp)$ is nonabelian. (We remark that in this setting the concept of a direct object corresponds to that of a direct cover as defined in the Foster census [4].)

When restricting to a specific subclass of graphs (and/or specific regular covering projections), the characterisation of irreducible morphisms, let alone direct ones, largely depends on the chosen subclass - as taking a quotient by the action of a semiregular group may result in a graph (and/or a morphism) outside that subclass; on one hand, some of the irreducible morphisms might not be included in the class at all, and on the other hand, a previously reducible projection might become irreducible. For instance, within the class of cubic arc-transitive graphs and regular covering projections there exist irreducible morphisms, even direct ones such as $\mathbf{F 8} \rightarrow \mathbf{F 2}$, with a nonsimple group as the group of covering transformations.

When speaking of cubic semisymmetric graphs it is necessary to consider all cubic edge-transitive graphs as objects and a certain subclass of regular covering projections as morphisms. This needs further explanation. First, note that there are semisymmetric cubic graphs which have a regular covering projection onto a smaller cubic arc-transitive graph (for example, the smallest one, the Gray graph S54, has a projection onto the Pappus graph F18). Second, as for the choice of morphisms we have restricted ourselves to consider only edge-transitive regular covering projections. By definition, a regular covering projection $\wp_{N}: X \rightarrow X_{N}$ is edge-transitive if at least one (minimal) edge-transitive subgroup of Aut $X$ projects along $\wp_{N}$ (implying that both graphs in question must be edge-transitive). By Proposition 3.1, $\wp_{N}$ is edge transitive if and only if $N$ is normalized by some (minimal) edge-transitive subgroup of Aut $X$; this is equivalent to requiring that $N$ is normal in some edge-transitive subgroup of Aut $X$ or, that the normalizer of $N$ within Aut $X$ is edgetransitive. In view of the above morphism restrictions it follows that an edge-transitive graph is basic whenever it has no edge-transitive covering projection onto a smaller edge-transitive graph, that is, if and only if no edge-transitive subgroup of Aut $X$ has a semiregular normal subgroup.

Note that each edge-transitive graph is a quotient graph arising from an edge-transitive covering projection (for instance, by taking an appropriate voltage assignment with values in the first homology group of the graph, the respective covering projection is edge-transitive). Therefore by restricting ourselves to edge-transitive covering projections, no basic graph (in the wider context of all regular covering projections) is lost; the set of basic graphs could just be larger. (No example is known to the authors, however, of a cubic edge-transitive graph covering another cubic edge-transitive graph with no covering projection between them being edge-transitive.)

Finally, we remark that in a later subsection we shall extend our class of morphisms to include all edge-transitive quotient projections. 
Excluding strongly reducible morphisms Clearly we would like to omit redundant morphisms (ideally, we would keep just the direct ones, up to isomorphism of projections) and even the unnecessary arc-transitive graphs, and still be able to generate all semisymmetric graphs starting from the set of basic graphs (by reconstructing each of the remaining covering projections in terms of voltages).

Recall that an edge-transitive regular covering projection $\wp_{N}: X \rightarrow X_{N}$ is reducible if there exists a decompostion $\wp_{N}=\wp_{N / K} \circ \wp_{K}$ where $\wp_{K}$ and $\wp_{N / K}$ are edge-transitive. In particular, $\wp_{N}$ is strongly reducible whenever there exists $K \triangleleft N$ such that some edge-transitive subgroup projects simultaneously along $\wp_{N}$ and $\wp_{K}$. Unfortunately, reducible projections which are not strongly reducible may exist.

Lemma 6.1. An edge-transitive regular covering projection $\wp_{N}: X \rightarrow X_{N}$ is strongly reducible if and only if there exists an edge-transitive group $H \leq$ Aut $X$ such that $N$ is normal but not a minimal normal subgroup of $H$.

By Lemma 6.1, it suffices to consider all edge-transitive subgroups $H \leq$ Aut $X$ and then take quotients by the action of all subgroups in $H$ which are minimal with respect to being normal and semiregular on $X$. Since each subgroup of a semiregular group is semiregular, these minimal subgroups are minimal normal subgroups of $H$ which are, in addition, semiregular on $X$. Moreover, we can consider edge-transitive subgroups and their minimal normal subgroups only up to conjugation in Aut $X$. Namely, normality and semiregularity are preserved under conjugation, and the projection relative to conjugate subgroups results in isomorphic covering projections. Now, if a selected subgroup $N$ is normal and not minimal normal in some other edge-transitive subgroup (where "some other" should be understood as relative to all edge-transitive subgroups, not just subgroups up to conjugation), then such a projection is discarded. Repeating the procedure recursively on all graphs thus obtained, no "smaller object below $X$ " is lost, and all morphisms are either irreducible, or at least not strongly reducible.

Strong reducibility can also be studied in terms of lifting edge-transitive subgroups along $\wp: Y \rightarrow X$ (usually given by means of a voltage assignment on $X$ ). Observe that if $\wp: Y \rightarrow X$ is a regular covering projection and if some (minimal) edge-transitive subgroup of $G \leq$ Aut $X$ lifts, then the lift $\widetilde{G} \leq$ Aut $Y$ is edge-transitive and hence $\wp$ is edge-transitive. The next lemma says that $\wp$ is strongly reducible if and only if there exists a decomposition $\wp=\wp_{N}=\wp_{N / K} \circ \wp_{K}$ and a (minimal) edge-transitive subgroup of Aut $X$ which lifts both along $\wp_{N}$ and $\wp_{N / K}$.

Lemma 6.2. An edge-transitive regular covering projection $\wp: Y \rightarrow X$ is strongly reducible if and only if there exists a minimal edge-transitive subgroup $G \leq$ Aut $X$ such that $\wp$ is $G$ admissible but not minimally $G$-admissible.

In view of Lemma 6.2, it suffices to consider all minimal edge-transitive subgroups $G \leq$ Aut $X$, and then find all minimally $G$-admissible covers of $X$ (up to isomorphism of covering projections). Moreover, we can consider minimal edge-transitive subgroups only up to conjugation in Aut $X$. Namely, if $G \leq$ Aut $X$ lifts along $\wp_{\zeta}: \operatorname{Cov}(X ; \zeta) \rightarrow X$ then a conjugate subgroup $G^{\alpha}$ lifts along an isomorphic covering projection $\zeta^{\alpha}$, defined by $\zeta^{\alpha}(a)=\zeta\left(\alpha^{-1}(a)\right)$ for each arc $a \in A(X)$. (Note that $G^{\alpha}$ might not lift along $\wp_{\zeta}$.) Repeating the procedure recursively on all larger graphs thus obtained, no "object above $X$ " is lost. (If there is an edge-transitive graph which covers $X$ but does not cover $X$ edge-transitively, then such a $\underline{\text { Springer }}$ 
graph is basic and needs to be found by other means.) All morphisms constructed this way are either irreducible, or at least not strongly reducible.

Characterising irreducible morphisms Let $\wp_{N}: X \rightarrow X_{N}$ be a reducible but not strongly reducible edge-transitive regular covering projection, and let $\wp_{N}=\wp_{N / K} \circ \wp_{K}$ be an edgetransitive depomposition. In such a case, edge-transitive subgroups of Aut $X_{K}$ projecting along $\wp_{N / K}$ are not related to edge-transitive-subgroups of Aut $X$ (via their projections along $\left.\wp_{K}\right)$. Similarly, no lift along $\wp_{N / K}$ of any edge-transitive group lifts along $\wp_{K}$. This makes the characterisation of irreducible morphisms rather difficult if not impossible.

We discuss the matter in some detail from the point of view of projecting groups first. Let $\mathcal{N}_{\text {min }}=\cup \operatorname{Min}_{\triangleleft} H$ be the set of all minimal normal subgroups of all edge-transitive subgroups $H \leq$ Aut $X$, and let nInc denote the "normal subgroup" relation. Observe that the set of nInc-minimal elements

$$
\mathcal{M}=\min _{\text {nInc }} \bigcup_{\text {H edge-transitive }} \operatorname{Min}_{\triangleleft} H
$$

can also be described as the set of all nInc-minimal elements in the set of all subgroups of Aut $X$ which are normalized by some minimal edge-transitive group, that is,

$$
\mathcal{M}=\min _{\mathrm{nInc}}\{M \mid M \text { is normalized by a minimal edge-transitive subgroup }\} \text {. }
$$

After calculation of the nInc-minimal elements, the set $\mathcal{M}$ can be reduced up to conjugationbut not before. Now, if $N \in \mathcal{M}$ acts semiregularly on the graph, then $\wp_{N}$ is edge-transitive and irreducible. However, the set

$$
\min _{\mathrm{nInc}}\left\{\mathcal{N}_{\min } \backslash \mathcal{M}\right\}
$$

may contain groups, acting semiregularly on $X$, which do not give rise to a strongly reducible projection. To decide whether such a projection $\wp_{N}$ is reducible or not, one has to consider $K \triangleleft N, K \in \mathcal{M}$ and the quotient graph $X_{K}$. Therefore, by restricting just to groups in $\mathcal{M}$, the reduction has gone too far. This can happen even in such special cases where Aut $X$ has a single conjugacy class of minimal edge-transitive subgroups.

Specialising further we now consider the case where Aut $X$ has a unique minimal edgetransitive subgroup (which is necessarily normal in Aut $X$ ). In such a case, an edge-transitive regular covering projection is either irreducible or strongly reducible, implying that irreducible projections can be characterised.

Lemma 6.3. Let $X$ be an edge-transitive graph such that Aut $X$ has a unique minimal edge-transitive subgroup, $H$. Let $\mathcal{M}$ be the set of all nInc-minimal elements

$$
\mathcal{M}=\min _{\mathrm{nInc}}\{M \mid \mathrm{M} \text { normalized by } \mathrm{H}\}=\min _{\mathrm{nInc}} \bigcup \operatorname{Min}_{\triangleleft} H^{\prime},
$$

where the union is taken over all subgroups $H^{\prime}$ of Aut $X$ containing $H$. Then the quotient projection byagroup $N \leq$ Aut $X$ is an irreducible edge-transitive regular covering projection if and only if $N$ is semiregular on $X$ and $N \in \mathcal{M}$.

Note that, in contrast with the general case, $\mathcal{M}$ now does contain all minimal normal subgroups of $H$ (if $H$ is not simple). The remaining groups in $\mathcal{M}$, if any, must intersect $H$ trivially.

As from the point of view of lifting groups we consider only the most particular case when Aut $X$ has a unique minimal edge-transitive subgroup, $H$. Like above, an edge-transitive 
regular covering projection $\wp: Y \rightarrow X$ is now either irreducible or strongly reducible. Hence $\wp$ is irreducible if and only if it is minimally $H$-admissible, by Lemma 6.2.

Our reduced list of direct covers Returning to our list, a further removal of many arctransitive graphs can be accomplished: if a graph $X$ is arc-transitive, we keep only those graphs below $X$ obtained by direct morphisms as found in the Foster census [4] (such morphisms are indeed edge-transitive). Moreover, there should be a semisymmetric cubic graph $Y$ on up to 768 vertices and an edge-transitive regular covering projection $Y \rightarrow X$.

As for the semisymmetric ones, recall that all such graphs in the list have been computed separately by other methods (so that we do have all the objects on up to 768 vertices). Checking their automorphism groups (using MAGMA [1]) reveals that, save for one single exceptionthe Gray graph - all have a unique minimal edge-transitive group which is, moreover, of index 1, 2, or 4 in the full automorphism group. As for the Gray graph, its minimal edgetransitive subgroups are precisely the Sylow 3-subgroups of its full automorphism group, and are thus pairwise conjugate.

The graphs F2, F4, F10, F28, F56b, F110, F182d, S110, S126, S182, S210, S504, S506 and $\mathbf{S 6 5 0}$ are basic, for they share the following property: no edge-transitive subgroup of the respective automorphism groups has a normal semiregular subgroup. This clearly holds for the arc-transitive ones, in view of the Foster census [4]. The minimal edge-transitive subgroup of $\mathbf{S 5 0 4}$ is its full automorphism group itself, isomorphic to $\operatorname{PSL}(2,8) \rtimes \mathbb{Z}_{3}$, and $\operatorname{PSL}(2,8)$ is its only proper nontrivial normal subgroup which is not semiregular since the respective quotient graph is $K_{1,3}$. The unique minimal edge-transitive subgroup $H$ for each of the remaining graphs $X$ is simple and of index at most 2 in Aut $X$. Therefore, a normal subgroup $N \triangleleft$ Aut $X$ either contains $H$ or intersects $H$ trivially. In the first case, $N$ is edgetransitive and hence not semiregular on $X$. In the second case, $N$ must be isomorphic to $\mathbb{Z}_{2}$. However, all these graphs are of the form twice an odd number, and so quotienting by $\mathbb{Z}_{2}$ cannot result in a cubic graph.

As for the irreducible morphisms, in the case of Gray graph we can take just the quotient by the minimal normal subgroup, isomorphic to $\mathbb{Z}_{3}$, of some Sylow 3-subgroup. Namely, since the Gray graph has order $54=2 \cdot 3^{3}$ and since cubic graphs have even order, the group by which we take the quotient must be a 3 -group, and hence contained in a minimal edgetransitive subgroup. The respective covering projection is $\mathbf{S 5 4} \rightarrow$ F18. All other irreducible morphisms are found using Lemma 6.3.

There are a few cases where $N \in \mathcal{M}$ intersects $H$ trivially. In this case $N$ must be a minimal normal subgroup of Aut $X$ and isomorphic to $\mathbb{Z}_{2}$. At most three such subgroups exist. This holds true because $H$ is normal and of index 1, 2 or 4 in Aut $X$. The only remaining possiblity that $N \cong \mathbb{Z}_{2}^{2}$ cannot occur for the following reason. If $N \cong \mathbb{Z}_{2}^{2}$ then $\mid$ Aut $X: H \mid=4$ and $N$ is a minimal normal subgroup of Aut $X$. But there is always a subgroup $H^{\prime} \leq$ Aut $X$ of index 2 containing $H$, and $N \cap H^{\prime}$ must be a proper and nontrivial subgroup of $N$. So $N \cap H^{\prime} \triangleleft$ Aut $X$, a contradiction to the minimality of $N$. Thus, $N \cong \mathbb{Z}_{2}$ is the only possibility. Clearly, $N$ is the only subgroup of order 2 complementary to $H$ in the group $N \times H$ (a subgroup of index 2 in Aut $X$ ). Hence $N$ is also normal in Aut $X$. As there are at most three subgroups of index 2 containing $H$, there are at most three minimal normal subgroups of Aut $X$ isomorphic to $\mathbb{Z}_{2}$ and having trivial intersection with $H$. However, a close inspection of our list shows that we only have cases where $H$ is of index 2 and $H$ is a simple group. Hence $N$ is always unique. These are the cases S220 $\rightarrow$ F110, S364 $\rightarrow$ F182d and S420 $\rightarrow$ S210. In all other cases, the group $N \in M$ is actually a minimal normal subgroup of $H$. There are never more than two such subgroups, and with a few exceptions, they are of coprime orders. Apart from $\mathbf{S 2 9 4} \rightarrow \mathbf{S 4 2}$, the minimal normal subgroups of $H$ are always normal in Aut $X$. 
Having found all irreducible morphisms, all graphs in the list can be constructed by a sequence of irreducible covering projections starting from the basic ones. Each semisymmetric graph projects onto at most two distinct smaller graphs in the list, and there are no nonisomorphic projections between the same pair of graphs in the list. Note that any composition of two or more irreducible morphisms is either a strongly reducible edge-transitive regular covering projection - or a covering projection which is not of our concern: a regular but not edge-transitive, or a nonregular covering projection.

We now show that all covering projections constructed as described above are actually direct. By the opening remarks at the beginning of this subsection, all morphisms between arctransitive graphs are direct. This trivially holds also in all cases where the group of covering transformations is of prime order. So what we need to check are the following projectionsthose with $\mid$ Aut $X: H \mid=1$ (namely $\mathbf{S 1 1 2} \rightarrow$ F14, S336a $\rightarrow$ F42, S400 $\rightarrow$ F16, S448 $\rightarrow$ F56a, S448 $\rightarrow$ S112, and S702a $\rightarrow$ F26), and those with $\mid$ Aut $X: H \mid=2$ (namely S144 $\rightarrow$ F16, S216 $\rightarrow$ S54, S432b $\rightarrow$ F48, S576 $\rightarrow$ F64, S576 $\rightarrow$ S144, and S768a $\rightarrow$ F192c), and finally, those with $\mid$ Aut $X: H \mid=4$ (namely S384 $\rightarrow$ F96b and S768b $\rightarrow$ F192a). All these covering projections arise as factorisations by a minimal normal subgroup of $H$.

If one of the above irreducible morphisms $X \rightarrow X_{N}$ is not direct, then there exists a subgroup $N^{\prime} \leq$ Aut $X$, acting semiregularly on $X$, such that $N^{\prime}$ (together with one of its normal subgroups) is normalized by $H$ and $X_{N^{\prime}} \cong X_{N}$. In particular, $\left|N^{\prime}\right|=|N|$.

Now, if any two minimal normal subgroups of $H$ have either equal or coprime cardinality, then $N^{\prime} \cap H=1$. So if $\mid$ Aut $X: H \mid \leq 2$, then $N^{\prime}$ is either trivial or isomorphic to $\mathbb{Z}_{2}$, a contradiction since $\wp_{N^{\prime}}$ was assumed strongly reducible. This allows us to dispose of the cases with $\mid$ Aut $X: H \mid=1$, except possibly for the projections $\mathbf{S 4 4 8} \rightarrow$ F56a and S448 $\rightarrow$ S112, and of the cases with $\mid$ Aut $X: H \mid=2$, except possibly for the projections $\mathbf{S 4 3 2 b} \rightarrow \mathbf{F 4 8}$ and S768a $\rightarrow$ F192c. We now consider these four projections separately.

The minimal normal subgroups of $H=$ Aut $\mathbf{S 4 4 8}$ are $N_{1} \cong \mathbb{Z}_{2}^{3}$ and $N_{2} \cong \mathbb{Z}_{2}^{2}$, giving rise to the two irreducible projections in the list. Clearly, no projection $\wp_{N^{\prime}}: \mathbf{S 4 4 8} \rightarrow \mathbf{S 1 1 2}$ is strongly reducible, for otherwise $H$ would contain a minimal normal subgroup isomorphic to $\mathbb{Z}_{2}$. So $\wp_{N_{2}}$ : S448 $\rightarrow \mathbf{S 1 1 2}$ is direct.

Suppose that $\wp_{N^{\prime}}: \mathbf{S 4 4 8} \rightarrow \mathbf{F 5 6 a}$ is strongly reducible. As $N^{\prime}$ is of order 8 and is not minimal normal in $H=$ Aut $\mathbf{S 4 4 8}$, it contains $N_{2}$. Thus, $\wp_{N^{\prime}}$ would factorise via $\wp_{N_{2}}$. But this is not possible because Aut S112 (the minimal edge-transitive group of S112) does not have a normal subgroup isomorphic to $\mathbb{Z}_{2}$ (as in fact its minimal normal subgroup is $\mathbb{Z}_{2}^{3}$ ). Consequently, $\wp_{N_{1}}: \mathbf{S 4 4 8} \rightarrow \mathbf{F 5 6 a}$ is direct.

Consider a strongly reducible projection $\wp_{N^{\prime}}: \mathbf{S 4 3 2} \mathbf{b} \rightarrow \mathbf{F 4 8}$. The group $N^{\prime}$ is of order 9 , and since $\mid$ Aut $X: H \mid=2$, the group $N^{\prime}$ is contained in $H$ and is not minimal normal in $H$. The minimal normal subgroups of $H$ are $N_{1} \cong \mathbb{Z}_{3}$ and $N_{2} \cong \mathbb{Z}_{3}^{2}$. Thus $N^{\prime}$ contains $N_{1} \cong \mathbb{Z}_{3}$, the group giving rise to the projection $\mathbf{S 4 3 2 b} \rightarrow \mathbf{S 1 4 4}$. The quotient group $N^{\prime} / N_{1} \cong \mathbb{Z}_{3}$ must be normalized by the minimal edge-transitive subgroup of Aut S144. But this minimal subgroup is also of index 2 in Aut $\mathbf{S 1 4 4}$, and must therefore contain the projection $N^{\prime} / N_{1}$. However, the minimal edge-transitive subgroup of Aut S144 has only one minimal normal subgroup, and this is isomorphic to $\mathbb{Z}_{3}^{2}$. Therefore, $\wp_{N_{2}}: \mathbf{S 4 3 2 b} \rightarrow \mathbf{F 4 8}$ is direct.

Consider a strongly reducible projection $\wp_{N^{\prime}}:$ S768a $\rightarrow$ F192c. The minimal normal subgroups of $H$ are $N_{1} \cong \mathbb{Z}_{2}$ and $N_{2} \cong \mathbb{Z}_{2}^{2}$. As $\left|N^{\prime}\right|=4$ and $\mid$ Aut $X: H \mid=2$, the group $N^{\prime}$ has a nontrivial intersection with $H$, implying that $\wp_{N^{\prime}}$ factorises through $\wp_{N_{1}}$ :S768a $\rightarrow$ S384. Thus Aut $\mathbf{S 3 8 4}$ has a group $N^{\prime} / N_{1} \cong \mathbb{Z}_{2}$ which is normalized by the minimal edge-transitive subgroup $H^{\prime} \leq$ Aut $\mathbf{S 3 8 4}$. The group $N^{\prime} / N_{1}$ is not contained in $H^{\prime}$ since the minimal normal subgroup of $H^{\prime}$ is isomorphic to $\mathbb{Z}_{2}^{2}$. But neither can $N^{\prime} / N_{1}$ have trivial intersection with $H^{\prime}$ for the following reason. First note that $H^{\prime}$ has index 4 in Aut S384, and that there would be 
a group $\mathbb{Z}_{2} \times H^{\prime}$ of index 2 in Aut $\mathbf{S 3 8 4}$ with a nontrivial centre. However, any of the three index 2 subgroups of Aut $\mathbf{S 3 8 4}$ are, according to MAGMA, centreless. This contradiction proves that $\wp_{N_{2}}: \mathbf{S 7 6 8 a} \rightarrow \mathbf{F 1 9 2 \mathbf { c }}$ is direct.

To complete the proof we need to discuss the two cases where $H$ is of index 4 in Aut $X$. First we consider a strongly reducible projection $\wp_{N^{\prime}}: \mathbf{S 3 8 4} \rightarrow \mathbf{F 9 6 b}$. Now, if $N^{\prime} \cap H=1$ then Aut $\mathbf{S 3 8 4}=N^{\prime} \times H$, and the centre of Aut $\mathbf{S 3 8 4}$ would be nontrivial. That is a contradiction, by MAGMA. We cannot have $N^{\prime} \cap H \cong \mathbb{Z}_{2}$ since the minimal normal subgroup of $H$ is $N=\mathbb{Z}_{2}^{2}$, and $N^{\prime} \leq H$ implies $N^{\prime}=N$, a contradiction. Thus, $\wp_{N}: \mathbf{S 3 8 4} \rightarrow \mathbf{F 9 6 b}$ is direct.

Finally, consider a strongly reducible projection $\wp_{N^{\prime}}: \mathbf{S 7 6 8 b} \rightarrow$ F192a. If $N^{\prime} \cap H=1$, then Aut $\mathbf{S 7 6 8 b}=N^{\prime} \times H$, a contradiction since the centre of Aut $\mathbf{S 7 6 8 b}$ coincides with that of $H$, by MAGMA. Now $N^{\prime} \cap H \neq 1$ implies that $\wp_{N^{\prime}}$ factorises through $\wp_{N_{1}}: \mathbf{S 7 6 8 b} \rightarrow \mathbf{S 3 8 4}$. But this would mean that the minimal edge-transitive subgroup of Aut $\mathbf{S 3 8 4}$ would normalize $N^{\prime} / N_{1} \cong \mathbb{Z}_{2}$, a contradiction since $\mathbf{S 3 8 4}$ has a unique irreducible projection, the one by the action of $\mathbb{Z}_{2}^{2}$. So $\wp_{N_{2}}$ :S768b $\rightarrow$ F192a is direct.

The above discussion is summarised in the following theorem.

Theorem 6.4. Our list displays, up to isomorphism of covering projections, all possible constructions of all cubic semisymmetric graphs on up to 768 vertices by taking successive direct regular covers of the following basic edge-transitive graphs: F2, F4, F10, F28, F56b, F110, F182d, S110, S126, S182, S210, S504, S506 and S650.

As was shown in [29], every finite cubic graph admitting an edge-transitive solvable group may be obtained from either the 3-dipole $\mathrm{Dip}_{3}$ (the graph F2), or the complete graph $K_{4}$ (the graph F4), by a sequence of elementary abelian covers. In other words, F2 and F4 are the only basic graphs needed to generate all finite edge-transitive cubic graphs having a solvable automorphism group. Thus each of the 21 cubic semisymmetric graphs in our list that has a solvable automorphism group can be obtained from one of these two graphs, as may be seen from our first diagram, see Figure 5.

The remaining 22 semisymmetric cubic graphs with a non-solvable automorphism group are displayed in Figures 6a and 6b. Note that every graph which reduces to F6 (the complete bipartite graph $K_{3,3}$ ) reduces also to $K_{1,3}$, see Figure 5. In Figures $6 \mathrm{a}$ and $6 \mathrm{~b}$, the eight

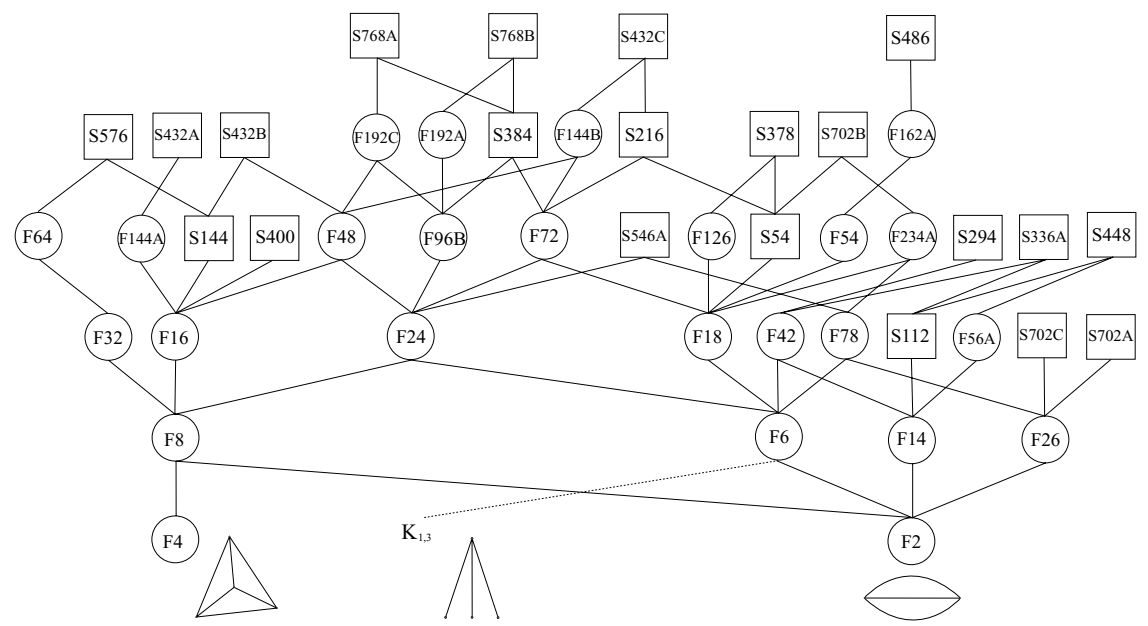

Fig. 5 Semisymmetric graphs with solvable automorphism group arising as covers of F2 and F4 


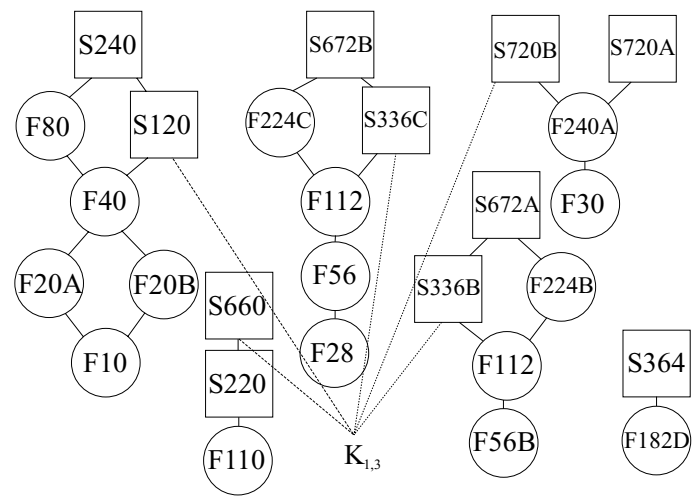

Fig. 6a The 11 semisymmetric graphs with nonsolvable automorphism group arising as covers of F10, F28, F56B, F110 and F182D

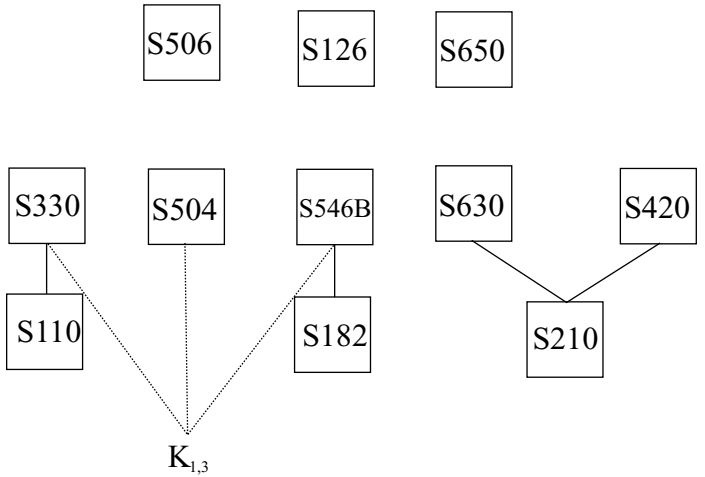

Fig. 6b The remaining 11 semisymmetric graphs with nonsolvable automorphism group

irreducible quotients of semisymmetric graphs onto $K_{1,3}$ are also displayed. These quotients are discussed below.

Quotients onto $K_{1,3}$ There are several examples of cubic semisymmetric graphs in our list having the tripod $K_{1,3}$ as a quotient, and as Section 5 demonstrates, they appear to be of particular interest. So let us enlarge our set of morphisms (and consequently, our set of objects). By definition, a quotient projection $q_{N}: X \rightarrow X_{N}$ is edge-transitive whenever at least one (minimal) edge-transitive subgroup of Aut $X$ projects along $q_{N}$. By Proposition 3.1, $q_{N}$ is edge-transitive if and only if $\operatorname{Ker} q_{N}$ is normalized by some (minimal) edge-transitive subgroup of Aut $X$.

As for the objects of our study, we note the following. If $N$ is edge-transitive, then the quotient graph is obviously isomorphic either to $K_{2}$ or to a one-vertex graph with a single loop or semiedge. Such quotients appear worthless and are exluded from further consideration. If $N$ is not edge-transitive then an edge-transitive quotient projection is either a regular covering projection, or else $X$ is bipartite and $X_{N} \cong K_{1,3}$. In view of these remarks, our extended class $\mathcal{C}$ of graphs and morphisms now includes: all edge-transitive cubic graphs as well as the graph $K_{1,3}$ as objects, and all edge-transitive regular covering projections together with all edge-transitive quotient projections onto $K_{1,3}$ as morphisms. The set of basic graphs 
consists of the graph $K_{1,3}$ and of all cubic edge-transitive graphs, which are basic relative to edge-transitive regular covering projections and do not have an edge-transitive quotient projection onto $K_{1,3}$. However, we still prefer to view all graphs that are basic in the old sense, as basic. (In the list on up to 768 vertices only the graph $\mathbf{S 5 0 4}$ is, formally, not basic anymore.)

When discussing (ir)reducibility of morphisms, the situation with non-covering quotient projections is subtler and more difficult to analyse because of certain anomalies which never occur with regular covering projections. In particular, a reducible quotient projection $q_{N}: X \rightarrow X_{N}$ might not arise as a strong decomposition. This anomaly can occur even if $q_{N}$ is strongly reducible (that is, one can have a decomposition $q_{N}=q_{M} \circ q_{K}$ where an edge transitive subgroup $H \leq$ Aut $X$ projects along $q_{N}$ and along $q_{K}$-and hence $\bar{H}=q_{K}^{*}(H)$ projects along $q_{M}$-however $N$ does not project along $q_{K}$ ). In our case the analysis is simplified to some extent — partly because noncovering quotients involve just a single graph, $K_{1,3}$, and partly because each semisymmetric graph $X$ on up to 768 vertices has a single minimal edge-transitive group (save for the Gray graph). But even this does not seem to be enough to obtain a characterisation of irreducible morphisms comparable to that of Lemma 6.3 (and worse, not even a characterisation of strongly reducible morphisms as in Lemma 6.1). The crucial additional information used in the analysis below is the fact that the minimal edgetransitive subgroup has a very small index in Aut $X$. Thus, we do not attempt to give a general approach to this problem (not even in the case of cubic graphs), and the special case of the Gray graph is treated separately.

Recall that the Gray graph has four minimal edge-transitive groups, namely the four Sylow 3 -subgroups of order 81 (thus acting regularly on the set of edges) having index 16 in the full automorphism group. Let $N$ be a subgroup giving rise to an edge-transitive quotient onto $K_{1,3}$. Then, as $N$ acts transitively on one bipartition set of 27 vertices, $|N|$ is divisible by 27. Now, a Sylow 3-subgroup of $N$ is entirely contained in some Sylow 3-subgroup $H$ of the full automorphism group, and must have order at least 27. So the Sylow 3-subgroup of $N$ is precisely $H \cap N$. As $N \neq H$ we have that $|H \cap N|=27$. Moreover, since $H \cap \operatorname{Ker} q_{N}$ cannot be $H$, we have $H \cap N=H \cap \operatorname{Ker} q_{N}$. Thus, $H \cap N$ is normal in $H$. The group $H \cap N$ cannot give rise to a covering projection for the respective quotient would be the dipole $\mathbf{F} 2$ (which does not have a graph morphism onto $K_{1,3}$ ). Consequently, quotienting by $H \cap N$ gives us $K_{1,3}$, and $q_{H \cap N}=q_{N}$. Now $H$ has a unique minimal normal subgroup $N_{0} \cong \mathbb{Z}_{3}$ (contained in $H \cap N$ ) which gives rise to a regular covering projection onto F18. So $q_{N}=q_{H \cap N / N_{0}} \circ q_{N_{0}}$ is an edge-transitive strong decomposition. Thus, no edge-transitive quotient of the Gray graph onto $K_{1,3}$ is irreducible.

Lemma 6.5. Let $X$ be an edge-transitive cubic graph having a unique minimal edgetransitive subgroup $H \leq$ Aut $X$ of index 1,2 or 4 in Aut $X$. If $q_{N}: X \rightarrow K_{1,3}$ is an irreducible edge-transitive quotient projection, then $q_{N}=q_{N_{0}}$, where $N_{0}$ is a minimal normal subgroup of $H$.

Proof: First observe that $N \cap H$ is nontrivial. For otherwise $N$ is a group of order at most 4 and hence $|V(X)| \leq 2|N| \leq 8$ (the first inequality follows from the fact that $N$ acts transitively on one part of the bipartition set of $X$ ). So $X$ is either $\mathbf{F 8}$ or $K_{3,3}$. Now, although $\mathbf{F 8}$ and $K_{3,3}$ do have $K_{1,3}$ as an epimorphic image, neither can be realised as an edge-transitive quotient by a subgroup outside the minimal edge-transitive group. Thus, $N \cap H$ is indeed nontrivial. Consider $N^{\prime}=\operatorname{Ker} q_{N} \cap H$. As $H$ normalizes $\operatorname{Ker} q_{N}$ we have $N^{\prime} \triangleleft H$. Also, $N^{\prime}$ is normal in $\operatorname{Ker} q_{N}$ (because $H$ is normal in Aut $X$ ) and hence $N$ normalizes $N^{\prime}$. Therefore, there exists a strong edge-transitive decomposition $q_{N}=q_{N / N \cap N^{\prime}} \circ q_{N^{\prime}}$. But $q_{N}$ is assumed 
irreducible, implying that $q_{N^{\prime}}=q_{N}$. Now let $N_{0}$ be a minimal normal subgroup of $H$ contained in $N^{\prime}$. Then $q_{N}=q_{N^{\prime}}=q_{N^{\prime} / N_{0}} \circ q_{N_{0}}$ is an edge-transitive strong decomposition. Since $q_{N}$ is assumed irreducible, the decomposition is trivial. Therefore, $q_{N}=q_{N_{0}}$.

There are eight semisymmetric graphs in our list having an edge-transitive quotient projection onto $K_{1,3}$ arising from a minimal normal subgroup $N$ of $H$; namely, the following: $\mathbf{S 1 2 0}$, S330, S336b, S336c, S504, S546b, S660 and S720b. Moreover, $N$ is uniquely determined since in all cases $H=\mathbb{Z}_{3} \times N$. Thus, $N$ is always normal in Aut $X$.

Note that if $N$ is a minimal normal subgroup of $H$ giving a quotient onto $K_{1,3}$, then $q_{N}$ might still be reducible (but it is unclear whether such a case exists or not). This is in striking contrast with the situation encountered in Lemma 6.3. Fortunately, nothing like that happens in the case of our finite list.

Lemma 6.6. Let $X$ be an edge-transitive cubic graph having a unique minimal edgetransitive subgroup $H \leq$ Aut $X$ of index 1, 2 or 4 in Aut $X$, and let $N$ be a minimal normal subgroup of index 3 in $H$ giving rise to an edge-transitive quotient projection $q_{N}: X \rightarrow K_{1,3}$. If $q_{N}$ is reducible, then there exists a strong edge-transitive decomposition $q_{N}=q_{N / N \cap L} \circ q_{L}$, where $L \cong \mathbb{Z}_{2}$ intersects $H$ trivially.

Proof: Let $K=\operatorname{Ker} q_{K}$ be a nontrivial semiregular subgroup contained in $\operatorname{Ker} q_{N}$ giving rise to an edge-transitive decomposition of $q_{N}$. Consider $N^{\prime}=\operatorname{Ker} q_{N} \cap H \triangleleft H$. Since $N \leq$ $N^{\prime} \leq \operatorname{Ker} q_{N}$ we have $q_{N^{\prime}}=q_{N}$ (and $N^{\prime}$ is actually the largest subgroup of $H$ with this property). Thus, $N^{\prime} \neq H$. So $|H: N|=3$ implies $N^{\prime}=N$. It follows that $H \cap K \leq H \cap$ $\operatorname{Ker} q_{N}=N$, and since $K$ is normalized by $H$ we have $H \cap K \triangleleft H$. But $N$ is a minimal normal subgroup of $H$. As $H \cap K$ is also semiregular on $X$ and hence not equal to $N$, we have $H \cap K=1$. Consequently, $K$ is a group of order 2 or 4 . Now, if $H=$ Aut $X$ then $K=1$, a contradiction. If $\mid$ Aut $X: H \mid=2$, let $L=K \cong \mathbb{Z}_{2}$. If $\mid$ Aut $X: H \mid=4$ then since $H$ is normal in Aut $X$, there is always a subgroup $H^{\prime}$ of index 2 in Aut $X$ which contains $H$ as an index 2 subgroup. In this case, let $L=K \cap H^{\prime} \cong \mathbb{Z}_{2}$. In both cases the group $L$ is normalized by $H$, and in particular, by $N$. So we do have an edge-transitive strong decomposition $q_{N}=$ $q_{N / N \cap L} \circ q_{L}$.

This lemma guarantees that, in our finite list, all quotients of semisymmetric graphs onto $K_{1,3}$ arising from minimal normal subgroups of $H$ are indeed irreducible. Namely, the only cases where there exists an edge-transitive regular covering projection by the action of a group $\mathbb{Z}_{2}$ intersecting the minimal edge-transitive subgroup trivially, are the cases $\mathbf{S 2 2 0} \rightarrow$ F110, $\mathbf{S 3 6 4} \rightarrow \mathbf{F 1 8 2 d}$ and $\mathbf{S 4 2 0} \rightarrow \mathbf{S 2 1 0}$. But in all these three cases the minimal edge-transitive subgroup is simple. Hence the respective graphs cannot have an edge-transitive quotient onto $K_{1,3}$.

There are other quotients onto $K_{1,3}$ in the list which are reducible. We now show that these cannot be defined on the semisymmetric graphs listed above, that is, all irreducible quotients in the list are indeed direct. So let $q_{N_{0}}: X \rightarrow K_{1,3}$ be an irreducible quotient projection, where $N_{0}$ is a minimal normal subgroup of index 3 in the minimal edge-transitive subgroup $H$ of Aut $X$. Further, let $q_{N}: X \rightarrow K_{1,3}$ be reducible. Consider $N^{\prime}=\operatorname{Ker} q_{N} \cap H$ which must be normal in $H$. If $N_{0} \leq N^{\prime}$ then $q_{N}=q_{N_{0}}$, a contradiction since $q_{N}$ is assumed reducible 
while $q_{N_{0}}$ is not. So $N^{\prime} \cap N=1$, implying that $N^{\prime} \cong \mathbb{Z}_{3}$. Hence $X$ is isomorphic to $K_{3,3}$, a contradiction.

The preceeding discussion is summarised in the following theorem.

Theorem 6.7. In our finite list, each semisymmetric graph having an irreducible edgetransitive quotient projection onto $K_{1,3}$ is a direct object over $K_{1,3}$.

\section{The list}

We are now ready to display the list of all connected cubic semisymmetric graphs on up to 768 vertices. For each graph in the list we have computed the order of the minimal edgetransitive group $H$. Recall that, except in the case of the Gray graph, this group is unique and characteristic in the full automorphism group. We have also computed (up to isomorphism of quotient/covering projections) all its direct quotient and covering projections. As explained in Section 6, these are found by taking the quotient relative to minimal normal subgroups of $H$ (also in the case of the Gray graph), except for the three cases where the quotient is taken by an action of $\mathbb{Z}_{2}$ outside $H$. For each projection we found the respective quotient graph, and the largest subgroup of the full automorphism group that projects. The use of MAGMA [1], and to some extent also the ATLAS [9], proved to be very helpful in our computations.

In summary the following information on these graphs is given in the list:

(i) the order;

(ii) the diameter;

(iii) the girth;

(iv) the order of the full automorphism group, as well as the order of the corresponding vertex stabilisers;

(v) the Goldschmidt type, together with additional relations which are satisfied by the generators of the automorphism group;

(vi) the two distance partitions with their refinements into orbits of the corresponding vertex stabilisers;

(vii) the order of a minimal edge-transitive subgroup (denoted by mET) with some additional information such as its solvability, simplicity, and isomorphism class;

(viii) all of its direct covers in the list, and the corresponding indices of the minimal edgetransitive subgroup in the maximal group that lifts;

(ix) all of its direct quotients (including the arc-transitive ones) and the maximal group that projects;

(x) possible membership of one of the families described in Section 7;

(xi) information on whether the graph is a basic semisymmetric graph, that is, a graph having no smaller regular graph as an edge-transitive quotient;

(xii) additional group relations which give, together with the Goldschmidt type, full information on the automorphism group of the graph and the stabilisers of two adjacent vertices.

The form of the information given in the list needs no explanation for items (i) to (v), (vii), (x) and (xi). The form in which the distance partitions (item (iv)) are given can be 
explained as follows. For a vertex $u$ of a graph $X$, let $N_{i}(u)$ denote the set of vertices which are at distance $i$ from $u$. Each such set $N_{i}(u)$ is clearly a union of orbits of the stabiliser (Aut $X)_{u}$, and sometimes just a single orbit. Let $\left\{n_{i, 1}, n_{i, 2}, \ldots, n_{i, t_{i}}\right\}$ be the set of lengths of the orbits of $N_{i}(u)$, and let $k_{i, j}$ denote the number of these orbits of length $n_{i, j}$, for $1 \leq j \leq t_{i}$. The distance partition of the graph $X$ relative to the vertex $u$ is then defined as the sequence

$$
\left(n_{0,1}^{k_{0,1}}, n_{1,1}^{k_{1,1}} \ldots n_{1, t_{i}}^{k_{1, t_{i}}}, \ldots, n_{d, 1}^{k_{d, 1}} \ldots n_{d, t_{i}}^{k_{d, t_{i}}}\right)
$$

where $d$ is the diameter of the graph $X$.

Clearly, any two vertices in the same orbit of Aut $X$ have identical distance partitions, and since the automorphism group of a semisymmetric graph has two orbits on vertices, two sequences are given for every graph in our list. If there are no vertices at distance $d$ from $u$ (which can occur if the longest geodesics begin and end in the orbit not containing $u$ ), then we take the last symbol in the above sequence to be 0. For example, in the case of the graph S112, it can be read from the sequences $\left(1^{1}, 3^{1}, 3^{2}, 3^{4}, 3^{8}, 1^{1} 3^{11}, 3^{8}, 1^{1} 3^{2}, 1^{1}\right)$ and $\left(1^{1}, 3^{1}, 3^{2}, 3^{4}, 3^{8}, 1^{1} 3^{11}, 1^{1} 3^{8}, 1^{1} 3^{2}, 0\right)$ that the number of vertices at distance 6 from a given vertex $u$ is either $8 \cdot 3=24$ or $8 \cdot 3+1 \cdot 1=25$. The vertex stabiliser (Aut $X)_{u}$ has 8 orbits of length 3 on $N_{6}(u)$ in the first case, and has 8 orbits of length 3 and one of length 1 in the second case.

Information regarding direct covers and direct quotients (items (viii) and (ix)) for each graph $X$ in our list is given in a table with three sections: a top section, a middle section, and a bottom section, with the middle section containing only the name of the graph $X$.

The bottom section is divided by vertical lines into several subsections, one for each of the direct quotients of the graph $X$. (If there are no direct quotients, that is, if $X$ is a basic graph, then the bottom section is omitted, and if there is exactly one direct quotient, there is no division into subsections.) Each subsection is again divided into an upper and lower part. The lower part gives the name of the quotient graph, which can be either a semisymmetric cubic graph (appearing elsewhere in the list), or a symmetric cubic graph (appearing in [7]), or $K_{1,3}$. In the upper part, three pieces of information are given. First, the symbol "Cov" is used to indicate that the quotient projection is a regular covering projection, and otherwise "Quo" is used to indicate that it is not. The second piece of information refers to the largest possible subgroup of Aut $X$ that projects along the quotient projection; "Aut $X$ " is written if this is the full automorphism group Aut $X$ itself, and otherwise only the index of the minimal edge-transitive group of $X$ in this maximal subgroup is given, and denoted by [- : mET]. The third piece of information is a description of the subgroup with respect to which the quotient projection is defined.

Similarly the top section is divided by vertical lines into several subsections, one for each graph in the list which is a direct cover of $X$. The upper part of each subsection gives the name of the covering graph, while the lower part gives two pieces of information: first, either "Aut $X$ " if Aut $X$ lifts along the covering projection, or otherwise the index $\left.\right|_{-}: \operatorname{mET} \mid$ of the minimal edge-transitive group in the largest subgroup of Aut $X$ that so lifts, and second, the voltage group (the group of covering transformations). If there are no such covering graphs in our list, then this is indicated by two sets of three dots. 
S54 (The Gray graph), families III and VII

G-Type: $G_{2}^{4} \quad$ additional relations: $x y x \bar{y} \bar{x} y \bar{x} \bar{y}$

Diam $X=6, \quad$ Girth $X=8$

$\mid$ Aut $X \mid=1296=2^{4} \cdot 3^{4}$

$\mid(\text { Aut } X)_{u} \mid=48=2^{4} \cdot 3$

Stab-Part:

$\left(1^{1}, 3^{1}, 6^{1}, 12^{1}, 4^{1} 12^{1}, 12^{1}, 4^{1}\right)$

$\left(1^{1}, 3^{1}, 6^{1}, 12^{1}, 12^{1}, 12^{1}, 8^{1}\right)$

$|\mathrm{mET}|=81$, solvable

(The four Syl 3-subgroups)

\begin{tabular}{|c|c|c|}
\hline S216 & S378 & S702b \\
\hline $\begin{array}{c}\text { : }: \mathrm{mET} \mid \\
\mathbb{Z}_{2}^{2} \\
\end{array}$ & $\begin{array}{c}\text { - }: \mathrm{mET} \mid \\
\mathbb{Z}_{7}\end{array}$ & $\begin{array}{c}\left.\right|_{-}: \operatorname{mET} \mid=1 \\
\mathbb{Z}_{13}\end{array}$ \\
\hline \multicolumn{3}{|c|}{$X=\mathbf{S 5 4}$} \\
\hline \multicolumn{3}{|c|}{$\begin{array}{c}\text { Cov } \\
\mid-: \mathrm{mET}^{\prime}=4 \\
\mathbb{Z}_{3} \\
\end{array}$} \\
\hline \multicolumn{3}{|c|}{ F18 } \\
\hline
\end{tabular}

S110, family II, basic

G-Type: $G_{2}^{1} \quad$ additional relations: $c d(\bar{x} \bar{y})^{2} x \bar{y}(\bar{x} \bar{y})^{2}$

Diam $X=7, \quad$ Girth $X=10$

$\mid$ Aut $X \mid=1320=2^{3} \cdot 3 \cdot 5 \cdot 11, \operatorname{PGL}(2,11)$

$\mid(\text { Aut } X)_{u} \mid=2^{3} \cdot 3$

StabPart:

$\left(1^{1}, 3^{1}, 6^{1}, 12^{1}, 12^{2}, 12^{1} 24^{1}, 12^{2}, 4^{1}\right)$

$\left(1^{1}, 3^{1}, 6^{1}, 12^{1}, 24^{1}, 12^{1} 24^{1}, 4^{1} 8^{1} 12^{1}, 4^{1}\right)$

\begin{tabular}{|c|}
\hline \hline S330 \\
\hline \hline Aut $X$ \\
$\mathbb{Z}_{3}$ \\
\hline$X=$ S110 \\
\hline \hline
\end{tabular}

$|\mathrm{mET}|=660$, simple, $\operatorname{PSL}(2,11)$

S112, family IV

G-Type: $G_{1} \quad$ additional relations: $\quad x y x \bar{y} x y(\bar{x} \bar{y})^{2}$

Diam $X=8, \quad$ Girth $X=10$

$\mid$ Aut $X \mid=168=2^{3} \cdot 3 \cdot 7$

$\mid(\text { Aut } X)_{u} \mid=3$

Stab-Part:

$\left(1^{1}, 3^{1}, 3^{2}, 3^{4}, 3^{8}, 1^{1} 3^{11}, 3^{8}, 1^{1} 3^{2}, 1^{1}\right)$

$\left(1^{1}, 3^{1}, 3^{2}, 3^{4}, 3^{8}, 1^{1} 3^{11}, 1^{1} 3^{8}, 1^{1} 3^{2}, 0\right)$

$|\mathrm{mET}|=168$, solvable

\begin{tabular}{|c|c|}
\hline \hline S336a & S448 \\
\hline \hline Aut $X$ & $\operatorname{Aut} X$ \\
$\mathbb{Z}_{3}$ & $\mathbb{Z}_{2}^{2}$ \\
\hline \hline \multicolumn{2}{|c|}{$X=\mathbf{S 1 1 2}$} \\
\hline \hline \multicolumn{2}{|c|}{ Cov } \\
Aut $X$ \\
$\mathbb{Z}_{2}^{3}$ \\
\hline \hline F14 \\
\hline \hline
\end{tabular}

\section{S120, family IIV}

G-Type: $G_{1}^{3}$ additional relations: $(x \bar{y} \bar{x} \bar{y})^{2}$

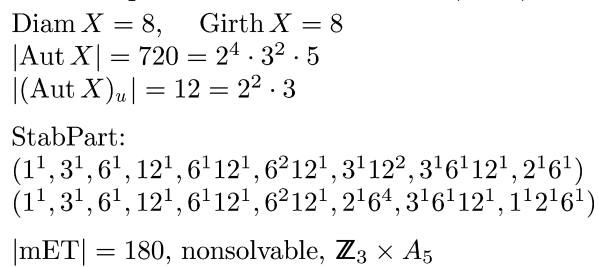

\begin{tabular}{|c|c|}
\hline \hline \multicolumn{2}{|c|}{ S240 } \\
\hline \multicolumn{2}{|c|}{ Aut $X$} \\
$\mathbb{Z}_{2}$ \\
\hline \hline \multicolumn{2}{|c|}{$X=\mathbf{S 1 2 0}$} \\
\hline \hline Cov & Quo \\
Aut $X$ & Aut $X$ \\
$\mathbb{Z}_{3}$ & $A_{5}$ \\
\hline \hline F40 & $K_{1,3}$ \\
\hline \hline
\end{tabular}


S126 (Tutte's 12-cage), basic

G-Type: $G_{4}^{1} \quad$ additional relations: $a^{3} b(x y)^{3}(\bar{x} \bar{y})^{3}$

Diam $X=6, \quad$ Girth $X=12$

$\mid$ Aut $X \mid=12096=2^{6} \cdot 3^{3} \cdot 7, U_{3}(3) \dot{2}$

$\mid(\text { Aut } X)_{u} \mid=192=2^{6} \cdot 3$

StabPart:

$\left(1^{1}, 3^{1}, 6^{1}, 12^{1}, 24^{1}, 48^{1}, 32^{1}\right)$

$\left(1^{1}, 3^{1}, 6^{1}, 12^{1}, 24^{1}, 48^{1}, 32^{1}\right)$

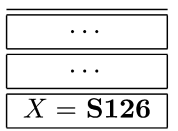

$|\mathrm{mET}|=6048$, simple, $U_{3}(3)$

S144, family V

G-Type: $G_{1}^{1} \quad$ additional relations: $(\bar{x} \bar{y})^{4}, x y \bar{x} y x y \bar{x} \bar{y} x \bar{y} \bar{x} \bar{y}$

Diam $X=8, \quad$ Girth $X=8$

$\mid$ Aut $X \mid=432=2^{4} \cdot 3^{3}$

$\mid(\text { Aut } X)_{u} \mid=6=2 \cdot 3$

Stab-Part:

$\left(1^{1}, 3^{1}, 6^{1}, 6^{2}, 3^{1} 6^{3}, 3^{2} 6^{5}, 3^{1} 6^{6}, 3^{3} 6^{2}, 1^{2} 3^{1}\right)$

$\left(1^{1}, 3^{1}, 6^{1}, 6^{2}, 3^{1} 6^{3}, 3^{2} 6^{5}, 1^{1} 2^{1} 3^{3} 6^{3}, 3^{3} 6^{2}, 2^{1} 6^{2}\right)$

$|\mathrm{mET}|=216$, solvable

\begin{tabular}{|c|c|}
\hline \hline S432b & S576 \\
\hline \hline Aut $X$ & Aut $X$ \\
$\mathbb{Z}_{3}$ & $\mathbb{Z}_{2}^{2}$ \\
\hline \hline \multicolumn{2}{|c|}{$X=$ S144 } \\
\hline \hline \multicolumn{2}{|c|}{ Cov } \\
Aut $X$ \\
$\mathbb{Z}_{3}^{2}$ \\
\hline \hline F16 \\
\hline \hline
\end{tabular}

S182, family II, basic

G-Type: $G_{2}^{1} \quad$ additional relations: $\quad(\bar{x} \bar{y})^{6}, c d \bar{x} y x \bar{y} \bar{x} \bar{y} \bar{x} y(\bar{x} \bar{y})^{2}$

Diam $X=8, \quad$ Girth $X=12$

$\mid$ Aut $X \mid=2184=2^{3} \cdot 3 \cdot 7 \cdot 13, P G L(2,13)$

$\mid(\text { Aut } X)_{u} \mid=24=2^{3} \cdot 3$

StabPart:

$\left(1^{1}, 3^{1}, 6^{1}, 12^{1}, 12^{2}, 24^{2}, 12^{2} 24^{1}, 4^{1} 12^{2}, 12^{1}\right)$

$\left(1^{1}, 3^{1}, 6^{1}, 12^{1}, 24^{2}, 24^{2}, 8^{1} 12^{2} 24^{1}, 4^{1} 12^{2}, 4^{1}\right)$

\begin{tabular}{|c|}
\hline \hline S546b \\
\hline \hline Aut $X$ \\
$\mathbb{Z}_{3}$ \\
\hline$X=$ S182 \\
\hline
\end{tabular}

$|\mathrm{mET}|=1092$, simple, $\operatorname{PSL}(2,13)$

S210, basic

G-Type: $G_{2}^{4} \quad$ additional relations: $\quad c d^{2} e \bar{x} \bar{y}(\bar{x} y \bar{x} \bar{y})^{2},(\bar{x} \bar{y})^{7}$

Diam $X=8, \quad$ Girth $X=10$

$\mid$ Aut $X \mid=5040=2^{4} \cdot 3^{2} \cdot 5 \cdot 7, S_{7}$

$\mid(\text { Aut } X)_{u} \mid=48=2^{4} \cdot 3$

Stab-Part:

$\left(1^{1}, 3^{1}, 6^{1}, 12^{1}, 12^{2}, 12^{1} 24^{1}, 12^{2} 24^{1}, 6^{1} 24^{2}, 2^{1} 24^{1}\right)$

$\left(1^{1}, 3^{1}, 6^{1}, 12^{1}, 24^{1}, 12^{1} 24^{1}, 6^{1} 48^{1}, 6^{1} 24^{2}, 8^{1} 12^{1}\right)$

\begin{tabular}{|c|c|}
\hline \hline S420 & S630 \\
\hline \hline Aut $X$ & Aut $X$ \\
$\mathbb{Z}_{2}$ & $\mathbb{Z}_{3}$ \\
\hline \hline \multicolumn{2}{|c|}{$X=$ S210 } \\
\hline
\end{tabular}

$|\mathrm{mET}|=2520$, simple, $A_{7}$

\section{S216}

G-Type: $G_{1}^{1} \quad$ additional relations: $(x y)^{3}(\bar{x} \bar{y})^{3},(x \bar{y} \bar{x} \bar{y})^{3}, x(y \bar{x})^{4} y x \bar{y} \bar{x} \bar{y}$

Diam $X=8, \quad$ Girth $X=12$

$\mid$ Aut $X \mid=648=2^{3} \cdot 3^{4}$

$\mid(\text { Aut } X)_{u} \mid=6=2 \cdot 3$

StabPart:

$\left(1^{1}, 3^{1}, 6^{1}, 6^{2}, 6^{4}, 6^{8}, 2^{1} 3^{2} 6^{10}, 3^{5} 6^{5}, 1^{2} 2^{2} 3^{1}\right)$

$\left(1^{1}, 3^{1}, 6^{1}, 6^{2}, 6^{4}, 6^{8}, 2^{1} 3^{2} 6^{10}, 3^{5} 6^{5}, 3^{3}\right)$

$|\mathrm{mET}|=324$, solvable

\begin{tabular}{|c|c|}
\hline \hline \multicolumn{2}{|c|}{ S432c } \\
\hline \hline \multicolumn{2}{|c|}{ Aut $X$} \\
$\mathbb{Z}_{2}$ \\
\hline \hline \multicolumn{2}{|c|}{$X=$ S216 } \\
\hline \hline Cov & Cov \\
Aut $X$ & Aut $X$ \\
$\mathbb{Z}_{3}$ & $\mathbb{Z}_{2}^{2}$ \\
\hline \hline F72 & S54 \\
\hline \hline
\end{tabular}




\section{$\mathrm{S} 220$}

G-Type: $G_{1}^{3} \quad$ additional relations: $\quad c \bar{x} \bar{y}(x \bar{y} \bar{x} \bar{y})^{2},(\bar{x} \bar{y})^{6}$

Diam $X=10, \quad$ Girth $X=10$

$\mid$ Aut $X \mid=1320=2^{3} \cdot 3 \cdot 5 \cdot 11$

$\mid(\text { Aut } X)_{u} \mid=12=2^{2} \cdot 3$

StabPart:

$\left(1^{1}, 3^{1}, 6^{1}, 12^{1}, 12^{2}, 6^{1} 12^{3}, 6^{3} 12^{3}, 2^{1} 6^{4} 12^{2}, 6^{2} 12^{1}, 3^{1}, 1^{1}\right)$

$\left(1^{1}, 3^{1}, 6^{1}, 12^{1}, 12^{2}, 6^{1} 12^{3}, 6^{5} 12^{2}, 2^{1} 6^{4} 12^{2}, 6^{4}, 3^{1}, 1^{1}\right)$

$|\mathrm{mET}|=660$, simple, $\operatorname{PSL}(2,11)$

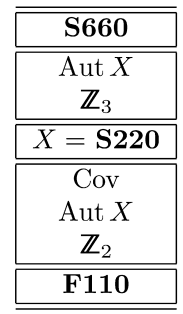

\section{$\mathrm{S} 240$}

G-Type: $G_{1}^{3} \quad$ additional relations: $\quad x \bar{y}(x y)^{2} \bar{x} y(\bar{x} \bar{y})^{2}, x y \bar{x} \bar{y} x \bar{y} \bar{x} y x \bar{y} \bar{x} \bar{y}$

Diam $X=10, \quad$ Girth $X=12$

$\mid$ Aut $X \mid=1440=2^{5} \cdot 3^{2} \cdot 5$

$\mid(\text { Aut } X)_{u} \mid=12=2^{2} \cdot 3$

StabPart:

$\left(1^{1}, 3^{1}, 6^{1}, 12^{1}, 12^{2}, 12^{4}, 6^{2} 12^{4}, 3^{1} 6^{2} 12^{3}, 1^{1} 2^{1} 12^{2}, 6^{1}, 2^{1}\right)$

$\left(1^{1}, 3^{1}, 6^{1}, 12^{1}, 12^{2}, 12^{4}, 4^{1} 6^{2} 12^{4}, 3^{1} 6^{2} 12^{3}, 1^{1} 2^{2} 6^{3}, 6^{1}, 2^{1}\right)$

$|\mathrm{mET}|=360$, nonsolvable

\begin{tabular}{|c|c|}
\hline \hline \multicolumn{2}{|c|}{$\cdots$} \\
\hline \hline \multicolumn{2}{|c|}{$\cdots$} \\
\hline \hline \multicolumn{2}{|c|}{$X=\mathbf{S 2 4 0}$} \\
Cov & Cov \\
$\mathbb{Z}_{2}$ & Aut $X$ \\
\hline \hline S120 & ․8 \\
\hline \hline
\end{tabular}

S294, family III

G-Type: $G_{1}^{2} \quad$ additional relations: $\quad(x \bar{y} \bar{x} \bar{y})^{3}, x y(\bar{x} y x \bar{y})^{2} \bar{x} \bar{y},(\bar{x} y \bar{x} \bar{y})^{3}$

$\operatorname{Diam} X=9, \quad$ Girth $X=12$

$\mid$ Aut $X \mid=882=2 \cdot 3^{2} \cdot 7^{2}$

$\mid(\text { Aut } X)_{u} \mid=6=2 \cdot 3$

StabPart:

$\left(1^{1}, 3^{1}, 3^{2}, 6^{2}, 6^{4}, 6^{8}, 3^{4} 6^{9}, 3^{4} 6^{10}, 1^{2} 3^{12} 6^{2}, 3^{2} 6^{1}\right)$

$\left(1^{1}, 3^{1}, 6^{1}, 6^{2}, 6^{4}, 6^{8}, 2^{1} 6^{12}, 3^{4} 6^{10}, 3^{2} 6^{6}, 3^{2} 6^{1}\right)$

$|\mathrm{mET}|=441$, solvable

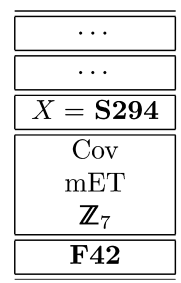

S330, family VII

G-Type: $G_{2}^{1} \quad$ additional relations: $\quad d^{2} \bar{x} y x y \bar{x} y(\bar{x} \bar{y})^{3}$

Diam $X=10, \quad$ Girth $X=12$

$\mid$ Aut $X \mid=3960=2^{3} \cdot 3^{2} \cdot 5 \cdot 11$

$\mid(\text { Aut } X)_{u} \mid=24=2^{3} \cdot 3$

StabPart:

$\left(1^{1}, 3^{1}, 6^{1}, 12^{1}, 12^{2}, 24^{2}, 12^{1} 24^{2}, 12^{2} 24^{2}, 6^{1} 12^{4}, 6^{1} 12^{2}, 2^{1} 6^{1} 12^{1}\right)$

$\left(1^{1}, 3^{1}, 6^{1}, 12^{1}, 24^{1}, 24^{2}, 8^{1} 12^{1} 24^{2}, 12^{2} 24^{2}, 12^{1} 24^{2}, 6^{1} 12^{2}, 2^{1} 4^{1}\right)$

$|\mathrm{mET}|=1980$, nonsolvable, $\mathbb{Z}_{3} \times \operatorname{PSL}(2,11)$

\begin{tabular}{|c|c|}
\hline \multicolumn{2}{|c|}{$\ldots$} \\
\hline \multicolumn{2}{|c|}{$\ldots$} \\
\hline \multicolumn{2}{|c|}{$X=\mathbf{S 3 3 0}$} \\
\hline Cov & Quo \\
\hline Aut $X$ & Aut $X$ \\
\hline $\mathbb{Z}_{3}$ & $\operatorname{PSL}(2,11)$ \\
\hline S110 & $K_{1,3}$ \\
\hline
\end{tabular}

\section{S336a}

G-Type: $G_{1} \quad$ additional relations: $(\bar{x} \bar{y})^{6},(x \bar{y} \bar{x} \bar{y})^{3}, x y(\bar{x} y x \bar{y})^{2} \bar{x} \bar{y}$

Diam $X=10, \quad$ Girth $X=12$

$\mid$ Aut $X \mid=504=2^{3} \cdot 3^{2} \cdot 7$

$\mid(\text { Aut } X)_{u} \mid=3$

StabPart:

$\left(1^{1}, 3^{1}, 3^{2}, 3^{4}, 3^{8}, 3^{16}, 3^{23}, 3^{26}, 1^{2} 3^{21}, 3^{9}, 1^{3}\right)$

$\left(1^{1}, 3^{1}, 3^{2}, 3^{4}, 3^{8}, 3^{16}, 1^{2} 3^{25}, 3^{26}, 3^{19}, 3^{9}, 1^{3}\right)$

$|\mathrm{mET}|=$ Aut $X$, solvable

\begin{tabular}{|c|c|}
\hline & \\
\hline & \\
\hline \multicolumn{2}{|c|}{$X=\mathbf{S 3 3 6 a}$} \\
\hline Cov & Cov \\
\hline Aut $X$ & Aut $X$ \\
\hline $\mathbb{Z}_{3}$ & $\mathbb{Z}_{2}^{3}$ \\
\hline S112 & F42 \\
\hline
\end{tabular}


S336b, family VII

G-Type: $G_{1}^{1} \quad$ additional relations: $\quad x \bar{y}(\bar{x} \bar{y})^{2} x \bar{y}(\bar{x} \bar{y})^{2}, \bar{x} y x \bar{y} x y \bar{x} \bar{y} \bar{x} y \bar{x} \bar{y}$

Diam $X=10, \quad$ Girth $X=12$

$\mid$ Aut $X \mid=1008=2^{4} \cdot 3^{2} \cdot 7$

$\mid(\text { Aut } X)_{u} \mid=6=2 \cdot 3$

StabPart:

$\left(1^{1}, 3^{1}, 6^{1}, 6^{2}, 6^{4}, 6^{8}, 2^{1} 3^{1} 6^{11}, 3^{4} 6^{12}, 2^{1} 3^{3} 6^{9}, 3^{1} 6^{3}, 1^{1}\right)$

$\left(1^{1}, 3^{1}, 6^{1}, 6^{2}, 6^{4}, 6^{8}, 3^{3} 6^{9}, 3^{4} 6^{12}, 2^{1} 3^{1} 6^{11}, 3^{1} 6^{3}, 3^{1}\right)$

$|\mathrm{mET}|=504$, nonsolvable, $\mathbb{Z}_{3} \times \operatorname{PSL}(2,7)$

\begin{tabular}{|c|c|}
\hline \hline \multicolumn{2}{|c|}{ S672a } \\
\hline \hline \multicolumn{2}{|c|}{ Aut $X$} \\
$\mathbb{Z}_{2}$ \\
\hline \hline \multicolumn{2}{|c|}{$X=$ S336b } \\
\hline \hline Cov & Quo \\
Aut $X$ & Aut $X$ \\
$\mathbb{Z}_{3}$ & PSL $(2,7)$ \\
\hline \hline F112a & $K_{1,3}$ \\
\hline \hline
\end{tabular}

S336c, family VII

G-Type: $G_{1}^{3} \quad$ additional relations: $\quad x y(\bar{x} y)^{2} x \bar{y}(\bar{x} \bar{y})^{2}, x \bar{y} \bar{x} \bar{y} \bar{x} y x \bar{y} x y(\bar{x} \bar{y})^{2}$

Diam $X=10, \quad$ Girth $X=12$

$\mid$ Aut $X \mid=2016=2^{5} \cdot 3^{2} \cdot 7$

$\mid(\text { Aut } X)_{u} \mid=12=2^{2} \cdot 3$

Stab-Part:

$\left(1^{1}, 3^{1}, 6^{1}, 12^{1}, 12^{2}, 12^{4}, 6^{1} 12^{6}, 6^{3} 12^{6}, 2^{1} 6^{7} 12^{1}, 3^{1} 6^{2}, 1^{1} 2^{1}\right)$

$\left(1^{1}, 3^{1}, 6^{1}, 12^{1}, 12^{2}, 12^{4}, 6^{1} 12^{6}, 6^{3} 12^{6}, 2^{1} 6^{3} 12^{3}, 3^{1} 6^{2}, 3^{1}\right)$

$|\mathrm{mET}|=504$, nonsolvable, $\mathbb{Z}_{3} \times \operatorname{PSL}(2,7)$

\begin{tabular}{|c|c|}
\hline \hline \multicolumn{2}{|c|}{ S672b } \\
\hline \hline \multicolumn{2}{|c|}{ Aut $X$} \\
$\mathbb{Z}_{2}$ \\
\hline \hline \multicolumn{2}{|c|}{$X=$ S336c } \\
\hline \hline Cov & Quo \\
Aut $X$ & Aut $X$ \\
$\mathbb{Z}_{3}$ & $P S L(2,7)$ \\
\hline \hline F112b & $K_{1,3}$ \\
\hline \hline
\end{tabular}

\section{S364}

G-Type: $G_{1}^{3} \quad$ additional relations: $\quad c(\bar{x} \bar{y})^{2}(x \bar{y})^{2}(\bar{x} \bar{y})^{2},(x y x \bar{y} \bar{x} \bar{y})^{2}$

Diam $X=12, \quad$ Girth $X=12$

$\mid$ Aut $X \mid=2184=2^{3} \cdot 3 \cdot 7 \cdot 13, \mathbb{Z}_{2} \times \operatorname{PSL}(2,13)$

$\mid(\text { Aut } X)_{u} \mid=12=2^{2} \cdot 3$

StabPart:

$\left(1^{1}, 3^{1}, 6^{1}, 12^{1}, 12^{2}, 12^{4}, 6^{1} 12^{5}, 6^{4} 12^{4}, 6^{3} 12^{4}, 2^{1} 6^{1} 12^{3}, 6^{1} 12^{1}, 3^{1}, 1^{1}\right)$

$\left(1^{1}, 3^{1}, 6^{1}, 12^{1}, 12^{2}, 12^{4}, 6^{3} 12^{4}, 6^{4} 12^{4}, 6^{5} 12^{3}, 2^{1} 6^{1} 12^{3}, 6^{1} 12^{1}, 3^{1}, 1^{1}\right)$

$|\mathrm{mET}|=1092$, simple, $\operatorname{PSL}(2,13)$

\begin{tabular}{|c|}
\hline \hline$\cdots$ \\
\hline \hline$\cdots$ \\
\hline \hline$X=$ S364 \\
\hline \hline $\operatorname{Cov}$ \\
Aut $X$ \\
$\mathbb{Z}_{2}=Z($ Aut $X)$ \\
\hline \hline F182d \\
\hline \hline
\end{tabular}

\section{S378}

G-Type: $G_{1} \quad$ additional relations: $\quad(x y)^{3}(\bar{x} \bar{y})^{3},(x \bar{y} \bar{x} \bar{y})^{3}, x(y \bar{x})^{2}(\bar{y} x)^{2}(y \bar{x})^{4} \bar{y}$

Diam $X=10, \quad$ Girth $X=12$

$\mid$ Aut $X \mid=567=3^{4} \cdot 7$

$\mid(\text { Aut } X)_{u} \mid=3$

StabPart:

$\left(1^{1}, 3^{1}, 3^{2}, 3^{4}, 3^{8}, 3^{16}, 1^{2} 3^{22}, 3^{23}, 3^{24}, 3^{19}, 3^{6}\right)$

$\left(1^{1}, 3^{1}, 3^{2}, 3^{4}, 3^{8}, 3^{16}, 1^{2} 3^{22}, 3^{23}, 3^{24}, 3^{19}, 1^{6} 3^{4}\right)$

$|\mathrm{mET}|=$ Aut $X$, solvable

\begin{tabular}{|c|c|}
\hline \hline \multicolumn{2}{|c|}{$\cdots$} \\
\hline \hline \multicolumn{2}{|c|}{$\cdots$} \\
\hline \hline \multicolumn{2}{|c|}{$X=\mathbf{S 3 7 8}$} \\
\hline \hline Cov & Cov \\
Aut $X$ & Aut $X$ \\
$\mathbb{Z}_{3}$ & $\mathbb{Z}_{7}$ \\
\hline \hline F126 & S54 \\
\hline \hline
\end{tabular}

\section{S384}

G-Type: $G_{1}^{3} \quad$ additional relations: $(\bar{x} \bar{y})^{6}, x y \bar{x} y x y \bar{x} \bar{y} x \bar{y} \bar{x} \bar{y}$

Diam $X=10, \quad$ Girth $X=12$

$\mid$ Aut $X \mid=2304=2^{8} \cdot 3^{2}$

$\mid(\text { Aut } X)_{u} \mid=12=2^{2} \cdot 3$

Stab-Part:

$\left(1^{1}, 3^{1}, 6^{1}, 12^{1}, 12^{2}, 12^{4}, 6^{3} 12^{4}, 6^{6} 12^{4}, 3^{1} 6^{8} 12^{3}, 3^{1} 6^{1} 12^{3}, 2^{1} 6^{1}\right)$

$\left(1^{1}, 3^{1}, 6^{1}, 12^{1}, 12^{2}, 12^{4}, 4^{1} 6^{3} 12^{4}, 6^{6} 12^{4}, 2^{1} 6^{4} 12^{4}, 3^{1} 6^{1} 12^{3}, 1^{1} 4^{1} 12^{1}\right)$

$|\mathrm{mET}|=576$, solvable

\begin{tabular}{|c|c|}
\hline \hline S768a & S768b \\
\hline \hline $\begin{array}{c}\text { Aut } X \\
\mathbb{Z}_{2}\end{array}$ & Aut $X$ \\
$\mathbb{Z}_{2}$ \\
\hline \hline \multicolumn{2}{|c|}{$X=$ S384 } \\
\hline \hline \multicolumn{2}{|c|}{ Cov } \\
Aut $X$ \\
$\mathbb{Z}_{2}^{2}$ \\
\hline \hline F96b \\
\hline \hline
\end{tabular}


S400, family $\mathbf{V}$

G-Type: $G_{1} \quad$ additional relations: $(\bar{x} \bar{y})^{4},(x \bar{y})^{6}, x y x \bar{y} \bar{x} y x(y \bar{x})^{2} \bar{y} x \bar{y} \bar{x} \bar{y}$

$\operatorname{Diam} X=10, \quad$ Girth $X=8$

$\mid$ Aut $X \mid=600=2^{3} \cdot 3 \cdot 5^{2}$

$\left|(\operatorname{Aut} X)_{u}\right|=3$

StabPart:

$\left(1^{1}, 3^{1}, 3^{2}, 3^{4}, 3^{7}, 3^{12}, 3^{20}, 3^{32}, 1^{1} 3^{36}, 1^{2} 3^{17}, 3^{1}\right)$

$\left(1^{1}, 3^{1}, 3^{2}, 3^{4}, 3^{7}, 3^{12}, 3^{20}, 3^{32}, 3^{36}, 1^{2} 3^{17}, 1^{1} 3^{1}\right)$

$|\mathrm{mET}|=\operatorname{Aut} X$, solvable

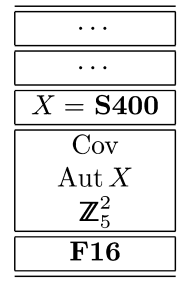

\section{$\mathbf{S} 420$}

G-Type: $G_{2}^{4} \quad$ additional relations: $\quad c d \bar{x} y \bar{x} \bar{y} x y x \bar{y} \bar{x} y \bar{x} \bar{y},(\bar{x} \bar{y})^{7}$

Diam $X=10, \quad$ Girth $X=12$

$\mid$ Aut $X \mid=10080=2^{5} \cdot 3^{2} \cdot 5 \cdot 7, \mathbb{Z}_{2} \times\left(\mathrm{mET} \rtimes \mathbb{Z}_{2}\right)$

$\mid(\text { Aut } X)_{u} \mid=48=2^{4} \cdot 3$

StabPart:

$\left(1^{1}, 3^{1}, 6^{1}, 12^{1}, 12^{2}, 24^{2}, 12^{1} 24^{3}, 12^{2} 24^{2} 48^{1}, 4^{1} 6^{1} 12^{1} 24^{3}, 3^{1} 24^{1}, 1^{1}\right)$

$\left(1^{1}, 3^{1}, 6^{1}, 12^{1}, 24^{1}, 24^{2}, 12^{1} 24^{1} 48^{1}, 12^{2} 24^{2} 48^{1}, 6^{1} 8^{1} 24^{1} 48^{1}, 3^{1} 24^{1}, 1^{1} 8^{1}\right)$

$|\mathrm{mET}|=2520$, simple, $A_{7}$

\begin{tabular}{|c|}
\hline \hline$\cdots$ \\
\hline \hline$\cdots$ \\
\hline \hline$X=\mathbf{S 4 2 0}$ \\
\hline \hline $\operatorname{Cov}$ \\
Aut $X$ \\
$\mathbb{Z}_{2}=Z($ Aut $X)$ \\
\hline \hline S210 \\
\hline
\end{tabular}

\section{S432a}

G-Type: $G_{1} \quad$ additional relations: $(x \bar{y} \bar{x} y)^{2}(\bar{x} \bar{y})^{3},(x y)^{2} \bar{x} y(x \bar{y})^{2}(\bar{x} \bar{y})^{2}$

Diam $X=10, \quad$ Girth $X=14$

$\mid$ Aut $X \mid=648=2^{3} \cdot 3^{4}$

$\left|(\operatorname{Aut} X)_{u}\right|=3$

StabPart:

$\left(1^{1}, 3^{1}, 3^{2}, 3^{4}, 3^{8}, 3^{16}, 3^{32}, 1^{2} 3^{39}, 1^{2} 3^{26}, 1^{1} 3^{11}, 3^{3}\right)$

$\left(1^{1}, 3^{1}, 3^{2}, 3^{4}, 3^{8}, 3^{16}, 3^{32}, 1^{2} 3^{39}, 1^{2} 3^{26}, 1^{1} 3^{11}, 3^{3}\right)$

$|\mathrm{mET}|=$ Aut $X$, solvable

\begin{tabular}{|c|}
\hline \hline$\cdots$ \\
\hline \hline$X=\mathbf{S 4 3 2 a}$ \\
\hline \hline Cov \\
Aut $X$ \\
$\mathbb{Z}_{3}$ \\
\hline \hline $\mathbf{F 1 4 4 a}$ \\
\hline \hline
\end{tabular}

\section{$\mathrm{S} 432 \mathrm{~b}$}

G-Type: $G_{1}^{1} \quad$ additional relations: $(y x)^{6}, x y \bar{x} y x y \bar{x} \bar{y} x \bar{y} \bar{x} \bar{y},(\bar{x} y)^{2} \bar{x} \bar{y} x y \bar{x} \bar{y} \bar{x} y(\bar{x} \bar{y})^{2}$

$\operatorname{Diam} X=10, \quad$ Girth $X=12$

$\mid$ Aut $X \mid=1296=2^{4} \cdot 3^{4}$

$\mid(\text { Aut } X)_{u} \mid=6=2 \cdot 3$

StabPart:

$\left(1^{1}, 3^{1}, 6^{1}, 6^{2}, 6^{4}, 6^{8}, 3^{1} 6^{12}, 3^{2} 6^{19}, 2^{3} 3^{1} 6^{15}, 3^{3} 6^{4}, 1^{2} 3^{1} 6^{1}\right)$

$\left(1^{1}, 3^{1}, 6^{1}, 6^{2}, 6^{4}, 6^{8}, 2^{2} 3^{1} 6^{12}, 3^{2} 6^{19}, 2^{4} 3^{3} 6^{14}, 3^{3} 6^{4}, 1^{1} 2^{2}\right)$

$|\mathrm{mET}|=648$, solvable

\begin{tabular}{|c|c|}
\hline \multicolumn{2}{|c|}{$\ldots$} \\
\hline \multicolumn{2}{|c|}{$\ldots$} \\
\hline \multicolumn{2}{|c|}{$X=\mathbf{S} 432 \mathrm{~b}$} \\
\hline Cov & $\mathrm{Cov}$ \\
\hline Aut $X$ & Aut $X$ \\
\hline $\mathbb{Z}_{3}$ & $\mathbb{Z}_{3}^{2}$ \\
\hline S144 & F48 \\
\hline
\end{tabular}

\section{S432c}

G-Type: $G_{1}^{1} \quad$ additional relations: $(x y)^{3}(\bar{x} \bar{y})^{3}, x(y \bar{x})^{4} y x \bar{y} \bar{x} \bar{y}$

Diam $X=12, \quad$ Girth $X=12$

$\mid$ Aut $X \mid=1296=2^{4} \cdot 3^{4}$

$\mid(\text { Aut } X)_{u} \mid=6=2 \cdot 3$

StabPart:

$\left(1^{1}, 3^{1}, 6^{1}, 6^{2}, 6^{4}, 6^{8}, 2^{1} 6^{13}, 3^{2} 6^{17}, 1^{2} 2^{4} 6^{14}, 3^{2} 6^{6}, 1^{2} 2^{1} 6^{1}, 3^{1}, 1^{1}\right)$

$\left(1^{1}, 3^{1}, 6^{1}, 6^{2}, 6^{4}, 6^{8}, 2^{1} 6^{13}, 3^{2} 6^{17}, 3^{4} 6^{14}, 3^{2} 6^{6}, 2^{1} 6^{1}, 3^{1}, 1^{1}\right)$

$|\mathrm{mET}|=648$, solvable

\begin{tabular}{|c|c|}
\hline \multicolumn{2}{|c|}{$\cdots$} \\
\hline \multicolumn{2}{|c|}{$\cdots$} \\
\hline \multicolumn{2}{|c|}{$X=\mathbf{S} 432 \mathrm{c}$} \\
\hline Cov & Cov \\
\hline Aut $X$ & Aut $X$ \\
\hline $\mathbb{Z}_{2}$ & $\mathbb{Z}_{3}$ \\
\hline S216 & F144b \\
\hline
\end{tabular}


S448, family IV

G-Type: $G_{1} \quad$ additional relations: $(\bar{x} \bar{y})^{6},(x \bar{y} \bar{x} \bar{y})^{3},\left(x(y \bar{x})^{2} \bar{y}\right)^{2}$

$\operatorname{Diam} X=10, \quad$ Girth $X=12$

$\mid$ Aut $X \mid=672=2^{5} \cdot 3 \cdot 7$

$\mid(\text { Aut } X)_{u} \mid=3$

StabPart:

$\left(1^{1}, 3^{1}, 3^{2}, 3^{4}, 3^{8}, 3^{16}, 3^{26}, 1^{1} 3^{36}, 3^{37}, 1^{1} 3^{17}, 1^{1} 3^{1}\right)$

$\left(1^{1}, 3^{1}, 3^{2}, 3^{4}, 3^{8}, 3^{16}, 3^{26}, 1^{1} 3^{36}, 3^{35}, 1^{1} 3^{17}, 1^{1} 3^{3}\right)$

$|\mathrm{mET}|=$ Aut $X$, solvable

\begin{tabular}{|c|c|}
\hline \hline \multicolumn{2}{|c|}{$\cdots$} \\
\hline \hline \multicolumn{2}{|c|}{$\cdots$} \\
\hline \hline \multicolumn{2}{|c|}{$X=\mathbf{S 4 4 8}$} \\
\hline \hline Cov & Cov \\
Aut $X$ & Aut $X$ \\
$\mathbb{Z}_{2}^{2}$ & $\mathbb{Z}_{2}^{3}$ \\
\hline \hline S112 & F56a \\
\hline \hline
\end{tabular}

\section{S486}

G-Type: $G_{1}^{1} \quad$ additional relations: $(x y)^{3}(\bar{x} \bar{y})^{3},(x \bar{y} \bar{x} \bar{y})^{3}, x(y \bar{x})^{7} y x \bar{y} \bar{x} \bar{y}$

Diam $X=12, \quad$ Girth $X=12$

$\mid$ Aut $X \mid=1458=2 \cdot 3^{6}$

$\mid(\text { Aut } X)_{u} \mid=6=2 \cdot 3$

StabPart:

$\left(1^{1}, 3^{1}, 6^{1}, 6^{2}, 6^{4}, 6^{8}, 2^{1} 3^{2} 6^{10}, 3^{3} 6^{10}, 3^{2} 6^{11}, 3^{1} 6^{13}, 3^{2} 6^{10}, 3^{4} 6^{3}, 3^{2}\right)$

$\left(1^{1}, 3^{1}, 6^{1}, 6^{2}, 6^{4}, 6^{8}, 2^{1} 3^{2} 6^{10}, 3^{3} 6^{10}, 3^{2} 6^{11}, 3^{1} 6^{13}, 3^{2} 6^{10}, 3^{4} 6^{3}, 3^{2}\right)$

$|\mathrm{mET}|=729$, solvable

\begin{tabular}{|c|}
\hline \hline$\cdots$ \\
\hline \hline$\cdots$ \\
\hline \hline$X=\mathbf{S 4 8 6}$ \\
\hline \hline Cov \\
Aut $X$ \\
$\mathbb{Z}_{3}$ \\
\hline \hline F162a \\
\hline \hline
\end{tabular}

S504, family VII, basic

G-Type: $G_{1}^{2} \quad$ additional relations: $(\bar{x} \bar{y})^{6}, c \bar{x} y x \bar{y} x y(x \bar{y})^{2}(\bar{x} \bar{y})^{2}$

Diam $X=10, \quad$ Girth $X=12$

$\mid$ Aut $X \mid=1512=2^{3} \cdot 3^{3} \cdot 7, \operatorname{PSL}(2,8) \rtimes \mathbb{Z}_{3}$

$\mid(\text { Aut } X)_{u} \mid=6=2 \cdot 3$

StabPart:

$\left(1^{1}, 3^{1}, 3^{2}, 6^{2}, 6^{4}, 6^{8}, 6^{15}, 6^{22}, 2^{1} 3^{6} 6^{17}, 3^{3} 6^{8}, 1^{2} 2^{2} 3\right)$

$\left(1^{1}, 3^{1}, 6^{1}, 6^{2}, 6^{4}, 6^{8}, 6^{15}, 6^{22}, 6^{19}, 3^{3} 6^{8}, 2^{1} 3^{1} 6^{2}\right)$

$|\mathrm{mET}|=$ Aut $X$, nonsolvable

\begin{tabular}{|c|}
\hline \hline$\cdots$ \\
\hline \hline$\cdots$ \\
\hline \hline$X=$ S504 \\
\hline \hline Quo \\
Aut $X$ \\
PSL $(2,8)$ \\
\hline \hline$K_{1,3}$ \\
\hline \hline
\end{tabular}

S506, family II, basic

G-Type: $G_{2}^{1} \quad$ additional relations: $c d^{2}(\bar{x} \bar{y})^{2}(x y)^{2}(\bar{x} \bar{y})^{2}$

Diam $X=11, \quad$ Girth $X=12$

$\mid$ Aut $X \mid=6072=2^{3} \cdot 3 \cdot 11 \cdot 23, \operatorname{PSL}(2,23)$

$\mid(\text { Aut } X)_{u} \mid=24=2^{4} \cdot 3$

StabPart:

$\left(1^{1}, 3^{1}, 6^{1}, 12^{1}, 12^{2}, 24^{2}, 12^{2} 24^{2}, 12^{2} 24^{3}, 6^{1} 12^{3} 24^{3}, 4^{1} 6^{1} 12^{2} 24^{2}, 12^{3}, 12^{1}\right)$

$\left(1^{1}, 3^{1}, 6^{1}, 12^{1}, 24^{1}, 24^{2}, 24^{3}, 12^{2} 24^{3}, 8^{1} 12^{3} 24^{3}, 4^{1} 6^{1} 12^{2} 24^{2}, 4^{1} 6^{1} 24^{1}, 12^{1}\right)$

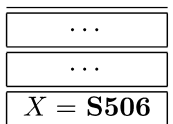

$|\mathrm{mET}|=$ Aut $X$, simple

S546a, family III

G-Type: $G_{1} \quad$ additional relations: $\quad(x \bar{y} \bar{x} \bar{y})^{3},(\bar{x} y \bar{x} \bar{y})^{3}, x \bar{y}(x y)^{2}(x \bar{y})^{2}(\bar{x} \bar{y})^{2}, x y x \bar{y} \bar{x} y x y \bar{x} \bar{y} x y(\bar{x} \bar{y})^{2}$

Diam $X=10, \quad$ Girth $X=12$

$\mid$ Aut $X \mid=819=3^{2} \cdot 7 \cdot 13$

$\mid(\text { Aut } X)_{u} \mid=3$

StabPart:

$\left(1^{1}, 3^{1}, 3^{2}, 3^{4}, 3^{8}, 3^{16}, 3^{28}, 3^{40}, 3^{44}, 3^{30}, 1^{2} 3^{8}\right)$

$\left(1^{1}, 3^{1}, 3^{2}, 3^{4}, 3^{8}, 3^{16}, 3^{28}, 3^{40}, 1^{2} 3^{46}, 3^{30}, 3^{6}\right)$

$|\mathrm{mET}|=$ Aut $X$, solvable

\begin{tabular}{|c|c|}
\hline \hline \multicolumn{2}{|c|}{$\cdots$} \\
\hline \hline \multicolumn{2}{|c|}{$X=$ S546a } \\
\hline \hline Cov & Cov \\
Aut $X$ & Aut $X$ \\
$\mathbb{Z}_{7}$ & $\mathbb{Z}_{13}$ \\
\hline \hline F78 & F42 \\
\hline \hline
\end{tabular}


S546b, family VII

G-Type: $G_{2}^{1} \quad$ additional relations: $(\bar{x} \bar{y})^{6}, c d x(y \bar{x})^{2} \bar{y} \bar{x} y x \bar{y} \bar{x} y(\bar{x} \bar{y})^{2}$

Diam $X=12, \quad$ Girth $X=12$

$\mid$ Aut $X \mid=6552=2^{3} \cdot 3^{2} \cdot 7 \cdot 13$

$\mid(\text { Aut } X)_{u} \mid=24=2^{3} \cdot 3$

StabPart:

$\left(1^{1}, 3^{1}, 6^{1}, 12^{1}, 12^{2}, 24^{2}, 12^{1} 24^{3}, 12^{2} 24^{5}, 12^{5} 24^{3}, 12^{3} 24^{1}, 6^{2} 12^{1}, 6^{1}, 2^{1}\right)$

$\left(1^{1}, 3^{1}, 6^{1}, 12^{1}, 24^{1}, 24^{2}, 12^{1} 24^{3}, 12^{2} 24^{5}, 8^{1} 12^{1} 24^{5}, 12^{3} 24^{1}, 4^{1} 12^{1}, 6^{1}, 2^{1}\right)$

$|\mathrm{mET}|=3276$, nonsolvable, $\mathbb{Z}_{3} \times \operatorname{PSL}(2,13)$

\begin{tabular}{|c|c|}
\hline & \\
\hline & \\
\hline \multicolumn{2}{|c|}{$X=\mathrm{S546b}$} \\
\hline $\mathrm{Cov}$ & Quo \\
\hline Aut $X$ & Aut $X$ \\
\hline $\mathbb{Z}_{3}$ & $\operatorname{PSL}(2,13)$ \\
\hline S182 & $K_{1,3}$ \\
\hline
\end{tabular}

\section{S576}

G-Type: $G_{1}^{1} \quad$ additional relations: $\quad(\bar{x} \bar{y})^{4},(x \bar{y})^{6},(x y \bar{x} \bar{y})^{4}, x \bar{y}(\bar{x} y)^{2} x \bar{y} x(y \bar{x})^{2} \bar{y} x \bar{y} \bar{x} \bar{y}$

Diam $X=12, \quad$ Girth $X=8$

$\mid$ Aut $X \mid=1728=2^{6} \cdot 3^{3}$

$\mid(\text { Aut } X)_{u} \mid=6=2 \cdot 3$

StabPart:

$\left(1^{1}, 3^{1}, 6^{1}, 6^{2}, 3^{1} 6^{3}, 3^{2} 6^{5}, 3^{2} 6^{9}, 3^{2} 6^{15}, 3^{3} 6^{22}, 3^{6} 6^{19}, 1^{2} 3^{2} 6^{8}, 3^{1} 6^{1}, 3^{1}\right)$

$\left(1^{1}, 3^{1}, 6^{1}, 6^{2}, 3^{1} 6^{3}, 3^{2} 6^{5}, 3^{2} 6^{9}, 3^{2} 6^{15}, 3^{3} 6^{22}, 3^{6} 6^{19}, 1^{1} 2^{2} 3^{3} 6^{7}, 3^{1} 6^{1}, 3^{1}\right)$

$|\mathrm{mET}|=864$, solvable

\begin{tabular}{|c|c|}
\hline \hline \multicolumn{2}{|c|}{$\cdots$} \\
\hline \hline \multicolumn{2}{|c|}{$\cdots$} \\
\hline \hline \multicolumn{2}{|c|}{$X=\mathbf{S 5 7 6}$} \\
\hline Cov & Cov \\
Aut $X$ & Aut $X$ \\
$\mathbb{Z}_{2}^{2}$ & $\mathbb{Z}_{3}^{2}$ \\
\hline \hline S144 & $\mathbf{F 6 4}$ \\
\hline \hline
\end{tabular}

\section{S630}

G-Type: $G_{2}^{4} \quad$ additional relations: $c d^{2} e \bar{x} \bar{y}(\bar{x} y \bar{x} \bar{y})^{2}$

Diam $X=14, \quad$ Girth $X=10$

$\mid$ Aut $X \mid=15120=2^{4} \cdot 3^{3} \cdot 5 \cdot 7$

$\mid(\text { Aut } X)_{u} \mid=$

StabPart:

$\left(1^{1}, 3^{1}, 6^{1}, 12^{1}, 12^{2}, 12^{1} 24^{1}, 12^{2} 24^{1}, 6^{1} 24^{1} 48^{1}, 6^{1} 48^{2}, 12^{1} 24^{2} 48^{1}\right.$,

$\left.24^{4}, 24^{3}, 12^{1} 24^{1}, 6^{1}, 2^{1}\right)$

$\left(1^{1}, 3^{1}, 6^{1}, 12^{1}, 24^{1}, 12^{1} 24^{1}, 6^{1} 48^{1}, 6^{1} 24^{1} 48^{1}, 12^{2} 24^{1} 48^{1}, 12^{1} 24^{2} 48^{1}\right.$,

$12^{2} 48^{2}, 24^{3}, 12^{1}, 6^{1}, 2^{1}$ )

$|\mathrm{mET}|=7560$, nonsolvable, $\mathbb{Z}_{3} \rtimes A_{7}$

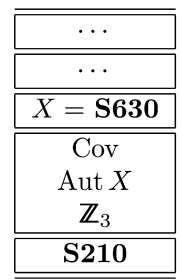

S650, basic

G-Type: $G_{2}^{4} \quad$ additional relations: $d \bar{x} \bar{y}(\bar{x} y \bar{x} \bar{y})^{2}$

Diam $X=13, \quad$ Girth $X=10$

$\mid$ Aut $X \mid=15600=2^{4} \cdot 3 \cdot 5^{2} \cdot 13, \mathrm{P} \Sigma \mathrm{L}(2,25)$

$\mid(\text { Aut } X)_{u} \mid=24=2^{4} \cdot 3$

StabPart:

$\left(1^{1}, 3^{1}, 6^{1}, 12^{1}, 12^{2}, 12^{1} 24^{1}, 12^{2} 24^{1}, 6^{1} 24^{1} 48^{1}, 6^{1} 48^{2}, 12^{1} 24^{2} 48^{1}, 12^{1} 24^{2} 48^{1}\right.$,

$\left.12^{1} 24^{1} 48^{1}, 12^{1} 24^{1}, 4^{1}\right)$

$\left(1^{1}, 3^{1}, 6^{1}, 12^{1}, 24^{1}, 12^{1} 24^{1}, 6^{1} 48^{1}, 6^{1} 24^{1} 48^{1}, 12^{2} 24^{1} 48^{1}, 12^{1} 24^{2} 48^{1}, 8^{1} 24^{3} 48^{1}\right.$,

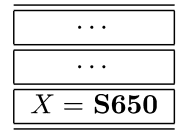

$\left.12^{1} 24^{1} 48^{1}, 4^{1} 12^{1}, 4^{1}\right)$

$|\mathrm{mET}|=7800$, simple, $\operatorname{PSL}(2,25)$

S660, family VII

G-Type: $G_{1}^{3} \quad$ additional relations: $(\bar{x} \bar{y})^{6}, d x \bar{y} \bar{x} \bar{y} x y \bar{x} y x \bar{y} \bar{x} \bar{y}$

Diam $X=12, \quad$ Girth $X=12$

$\mid$ Aut $X \mid=3960=2^{3} \cdot 3^{2} \cdot 5 \cdot 11$

$\mid(\text { Aut } X)_{u} \mid=12=2^{2} \cdot 3$

StabPart:

$\left(1^{1}, 3^{1}, 6^{1}, 12^{1}, 12^{2}, 12^{4}, 6^{3} 12^{5}, 6^{4} 12^{8}, 6^{5} 12^{11}, 6^{4} 12^{10}, 2^{1} 3^{1} 6^{1} 12^{4}, 3^{1}, 0\right)$

$\left(1^{1}, 3^{1}, 6^{1}, 12^{1}, 12^{2}, 12^{4}, 6^{1} 12^{6}, 6^{4} 12^{8}, 6^{5} 12^{11}, 6^{4} 12^{10}, 2^{2} 6^{3} 12^{3}, 3^{1}, 1^{1}\right)$

$|\mathrm{mET}|=1980$, nonsolvable, $\mathbb{Z}_{3} \times \operatorname{PSL}(2,11)$

\begin{tabular}{|c|c|}
\hline \hline \multicolumn{2}{|c|}{$\cdots$} \\
\hline \hline \multicolumn{2}{|c|}{$X=\mathbf{S 6 6 0}$} \\
\hline \hline \multicolumn{2}{|c|}{ Quo } \\
\hline \hline Cov & Aut $X$ \\
Aut $X$ & PSL $(2,11)$ \\
$\mathbb{Z}_{3}$ & $K_{1,3}$ \\
\hline \hline S220 \\
\hline \hline
\end{tabular}




\section{S672a}

G-Type: $G_{1}^{1} \quad$ additional relations: $\quad x y \bar{x} y(x \bar{y} \bar{x} \bar{y})^{2}, x \bar{y}(x y)^{3} \bar{x} y(\bar{x} \bar{y})^{3}$

Diam $X=12, \quad$ Girth $X=12$

$\mid$ Aut $X \mid=2016=2^{5} \cdot 3^{2} \cdot 7$

$\mid(\text { Aut } X)_{u} \mid=6=2 \cdot 3$

StabPart:

$\left(1^{1}, 3^{1}, 6^{1}, 6^{2}, 6^{4}, 6^{8}, 3^{2} 6^{11}, 3^{2} 6^{19}, 2^{1} 6^{26}, 3^{2} 6^{22}, 3^{2} 6^{11}, 3^{1} 6^{2}, 1^{1} 2^{1}\right)$

$\left(1^{1}, 3^{1}, 6^{1}, 6^{2}, 6^{4}, 6^{8}, 2^{1} 6^{13}, 3^{2} 6^{19}, 2^{1} 3^{4} 6^{25}, 3^{2} 6^{22}, 2^{1} 6^{9}, 3^{1} 6^{2}, 1^{1} 2^{2}\right)$

$|\mathrm{mET}|=1008$, nonsolvable

$\mathbb{Z}_{2} \operatorname{ext}_{\text {cent }}\left(\mathbb{Z}_{3} \times \operatorname{PSL}(2,7)\right) \cong \mathbb{Z}_{6} \operatorname{ext}_{\text {cent }} \operatorname{PSL}(2,7)$

\begin{tabular}{|c|c|}
\hline \hline \multicolumn{2}{|c|}{$\cdots$} \\
\hline \hline \multicolumn{2}{|c|}{$\cdots$} \\
\hline \hline \multicolumn{2}{|c|}{$X=\mathbf{S 6 7 2 a}$} \\
\hline \hline Cov & Cov \\
Aut $X$ & Aut $X$ \\
$\mathbb{Z}_{2}$ & $\mathbb{Z}_{3}$ \\
\hline \hline S336b & F224b \\
\hline \hline
\end{tabular}

\section{S672b}

G-Type: $G_{1}^{3} \quad$ additional relations: $\quad x y(\bar{x} y)^{2} x \bar{y}(\bar{x} \bar{y})^{2}$

Diam $X=14, \quad$ Girth $X=12$

$\mid$ Aut $X \mid=4032=2^{6} \cdot 3^{2} \cdot 7$

$\mid(\text { Aut } X)_{u} \mid=12=2^{2} \cdot 3$

StabPart:

$\left(1^{1}, 3^{1}, 6^{1}, 12^{1}, 12^{2}, 12^{4}, 6^{1} 12^{6}, 6^{2} 12^{9}, 2^{1} 6^{3} 12^{11}, 6^{2} 12^{10}, 2^{1} 4^{1} 6^{2} 12^{4}\right.$, $\left.6^{1} 12^{1}, 2^{1} 6^{1}, 3^{1}, 1^{1}\right)$

$\left(1^{1}, 3^{1}, 6^{1}, 12^{1}, 12^{2}, 12^{4}, 6^{1} 12^{6}, 6^{2} 12^{9}, 2^{1} 12^{12}, 6^{2} 12^{10}, 6^{2} 12^{5}\right.$,

$\left.6^{1} 12^{1}, 2^{1} 6^{1}, 3^{1}, 1^{1}\right)$

$|\mathrm{mET}|=1008$, nonsolvable

\begin{tabular}{|c|c|}
\hline \hline \multicolumn{2}{|c|}{$\cdots$} \\
\hline \hline \multicolumn{2}{|c|}{$\cdots$} \\
\hline \hline \multicolumn{2}{|c|}{$X=\mathbf{S 6 7 2 b}$} \\
\hline \hline Cov & Cov \\
Aut $X$ & Aut $X$ \\
$\mathbb{Z}_{2}$ & $\mathbb{Z}_{3}$ \\
\hline \hline S336c & F224c \\
\hline \hline
\end{tabular}

$\mathbb{Z}_{2} \operatorname{ext}_{\text {cent }}\left(\mathbb{Z}_{3} \times \operatorname{PSL}(2,7)\right) \cong \mathbb{Z}_{6} \operatorname{ext}_{\text {cent }} \operatorname{PSL}(2,7)$

\section{S702a}

G-Type: $G_{1} \quad$ additional relations: $(x \bar{y} \bar{x} \bar{y})^{3}, x y \bar{x} \bar{y} \bar{x} y x y(\bar{x} \bar{y})^{4}$

Diam $X=10, \quad$ Girth $X=12$

$\mid$ Aut $X \mid=1053=3^{4} \cdot 13$

$\mid(\text { Aut } X)_{u} \mid=3$

StabPart:

$\left(1^{1}, 3^{1}, 3^{2}, 3^{4}, 3^{8}, 3^{16}, 3^{30}, 3^{56}, 1^{2} 3^{66}, 3^{3} 3^{39}, 3^{10}\right)$

$\left(1^{1}, 3^{1}, 3^{2}, 3^{4}, 3^{8}, 3^{16}, 3^{30}, 3^{56}, 3^{64}, 1^{3} 3^{39}, 1^{2} 3^{12}\right)$

$|\mathrm{mET}|=$ Aut $X$, solvable

\begin{tabular}{|c|}
\hline \hline$\cdots$ \\
\hline \hline$\cdots$ \\
\hline \hline$X=$ S702a \\
\hline \hline Cov \\
Aut $X$ \\
$\mathbb{Z}_{3}^{3}$ \\
\hline \hline F26 \\
\hline \hline
\end{tabular}

\section{S702b}

G-Type: $G_{1} \quad$ additional relations: $\quad(x y)^{3}(\bar{x} \bar{y})^{3},(x \bar{y} \bar{x} \bar{y})^{3}, x(y \bar{x})^{2} \bar{y} x y \bar{x} \bar{y} x(y \bar{x})^{6} \bar{y}$

Diam $X=14, \quad$ Girth $X=12$

$\mid$ Aut $X \mid=1053=3^{4} \cdot 13$

$\mid(\text { Aut } X)_{u} \mid=3$

StabPart:

$\left(1^{1}, 3^{1}, 3^{2}, 3^{4}, 3^{8}, 3^{16}, 1^{2} 3^{22}, 3^{23}, 3^{24}, 3^{27}, 3^{30}, 3^{33}, 3^{28}, 3^{13}, 3^{2}\right)$

$\left(1^{1}, 3^{1}, 3^{2}, 3^{4}, 3^{8}, 3^{16}, 1^{2} 3^{22}, 3^{23}, 3^{24}, 3^{27}, 3^{30}, 3^{33}, 3^{28}, 3^{13}, 1^{6}\right)$

$|\mathrm{mET}|=$ Aut $X$, solvable

\begin{tabular}{|c|c|}
\hline \hline \multicolumn{2}{|c|}{$\cdots$} \\
\hline \hline \multicolumn{2}{|c|}{$\cdots$} \\
\hline \hline \multicolumn{2}{|c|}{$X=$ S702b } \\
\hline \hline Cov & Cov \\
Aut $X$ & Aut $X$ \\
$\mathbb{Z}_{3}$ & $\mathbb{Z}_{13}$ \\
\hline \hline F234a & S54 \\
\hline \hline
\end{tabular}

\section{S702c}

G-Type: $G_{1} \quad$ additional relations: $(x y)^{2} \bar{x} \bar{y} x y \bar{x} y(\bar{x} \bar{y})^{2}$

Diam $X=11, \quad$ Girth $X=14$

$\mid$ Aut $X \mid=1053=3^{4} \cdot 13$

$\mid(\text { Aut } X)_{u} \mid=3$

StabPart:

$\left(1^{1}, 3^{1}, 3^{2}, 3^{4}, 3^{8}, 3^{16}, 3^{32}, 3^{57}, 3^{68}, 1^{2} 3^{38}, 1^{2} 3^{6}, 1^{1}\right)$

$\left(1^{1}, 3^{1}, 3^{2}, 3^{4}, 3^{8}, 3^{16}, 3^{32}, 3^{57}, 1^{2} 3^{66}, 1^{2} 3^{38}, 3^{8}, 1^{1}\right)$

$|\mathrm{mET}|=$ Aut $X$, solvable

\begin{tabular}{|c|}
\hline \hline$\cdots$ \\
\hline \hline$\cdots$ \\
\hline \hline$X=$ S702c \\
\hline \hline $\operatorname{Cov}$ \\
Aut $X$ \\
$\mathbb{Z}_{3}^{3}$ \\
\hline \hline F26 \\
\hline \hline
\end{tabular}




\section{S720a}

G-Type: $G_{1} \quad$ additional relations: $\quad x y(\bar{x} y)^{2} x \bar{y}(\bar{x} \bar{y})^{3}, x \bar{y} \bar{x} \bar{y}(x y)^{2} \bar{x} \bar{y} \bar{x} y(\bar{x} \bar{y})^{2}$

Diam $X=10, \quad$ Girth $X=14$

$\mid$ Aut $X \mid=1080=2^{3} \cdot 3^{3} \cdot 5, \mathbb{Z}_{3} \operatorname{ext}_{\text {cent }} A_{6}$

$\mid(\text { Aut } X)_{u} \mid=3$

StabPart:

$\left(1^{1}, 3^{1}, 3^{2}, 3^{4}, 3^{8}, 3^{16}, 3^{32}, 3^{57}, 1^{4} 3^{64}, 3^{42}, 1^{1} 3^{12}\right)$

$\left(1^{1}, 3^{1}, 3^{2}, 3^{4}, 3^{8}, 3^{16}, 3^{32}, 3^{57}, 1^{2} 3^{66}, 3^{42}, 1^{3} 3^{10}\right)$

$|\mathrm{mET}|=$ Aut $X$, nonsolvable

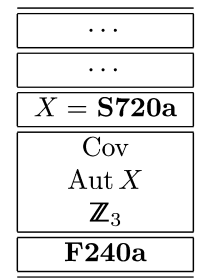

S720b, family VII

G-Type: $G_{1}^{1} \quad$ additional relations: $\quad x y(\bar{x} y)^{2} x \bar{y}(\bar{x} \bar{y})^{3}, x y x \bar{y} x y \bar{x} \bar{y} x \bar{y} \bar{x} y(\bar{x} \bar{y})^{2}$

Diam $X=12, \quad$ Girth $X=14$

$\mid$ Aut $X \mid=2160=2^{4} \cdot 3^{3} \cdot 5$

$\mid(\text { Aut } X)_{u} \mid=6=2 \cdot 3$

StabPart:

$\left(1^{1}, 3^{1}, 6^{1}, 6^{2}, 6^{4}, 6^{8}, 6^{16}, 3^{1} 6^{28}, 2^{2} 3^{3} 6^{29}, 3^{5} 6^{18}, 2^{2} 3^{4} 6^{5}, 3^{1}, 0\right)$

$\left(1^{1}, 3^{1}, 6^{1}, 6^{2}, 6^{4}, 6^{8}, 6^{16}, 3^{1} 6^{28}, 2^{4} 3^{1} 6^{30}, 3^{5} 6^{18}, 2^{4} 3^{5} 6^{3}, 3^{1}, 1^{1}\right)$

$|\mathrm{mET}|=1080$, nonsolvable, $\mathbb{Z}_{3} \times A_{6}$

\begin{tabular}{|c|c|}
\hline \multicolumn{2}{|c|}{$\ldots$} \\
\hline \multicolumn{2}{|c|}{$\cdots$} \\
\hline \multicolumn{2}{|c|}{$X=\mathbf{S 7 2 0 b}$} \\
\hline Cov & Quo \\
\hline Aut $X$ & Aut $X$ \\
\hline $\mathbb{Z}_{3}$ & $A_{6}$ \\
\hline F240a & $K_{1,3}$ \\
\hline
\end{tabular}

\section{S768a}

G-Type: $G_{1}^{1} \quad$ additional relations: $\quad(\bar{x} \bar{y})^{6}, x y \bar{x} y x y \bar{x} \bar{y} x \bar{y} \bar{x} \bar{y}, x \bar{y} x y(x \bar{y})^{2}(\bar{x} y)^{3} \bar{x} \bar{y}$

Diam $X=12, \quad$ Girth $X=12$

$\mid$ Aut $X \mid=2304=2^{8} \cdot 3^{2}$

$\mid(\text { Aut } X)_{u} \mid=6=2 \cdot 3$

StabPart:

$\left(1^{1}, 3^{1}, 6^{1}, 6^{2}, 6^{4}, 6^{8}, 3^{1} 6^{12}, 3^{2} 6^{19}, 3^{2} 6^{27}, 3^{3} 6^{29}, 2^{2} 3^{2} 6^{16}, 3^{2} 6^{2}, 1^{1} 3^{1}\right)$

$\left(1^{1}, 3^{1}, 6^{1}, 6^{2}, 6^{4}, 6^{8}, 2^{2} 3^{1} 6^{12}, 3^{2} 6^{19}, 2^{1} 3^{2} 6^{26}, 3^{3} 6^{29}, 1^{1} 2^{5} 3^{1} 6^{15}, 3^{2} 6^{2}, 1^{2} 2^{2}\right)$

$|\mathrm{mET}|=1152$, solvable

\begin{tabular}{|c|c|}
\hline & \\
\hline & \\
\hline \multicolumn{2}{|c|}{$X=\mathbf{S 7 6 8 a}$} \\
\hline Cov & $\mathrm{Cov}$ \\
\hline $\begin{array}{c}\text { Aut } X \\
\mathbb{Z}_{2}\end{array}$ & $\begin{array}{c}\text { Aut } X \\
\mathbb{Z}_{2}^{2}\end{array}$ \\
\hline S384 & S192c \\
\hline
\end{tabular}

\section{S768b}

G-Type: $G_{1}^{3} \quad$ additional relations: $\quad x y \bar{x} y x y \bar{x} \bar{y} x \bar{y} \bar{x} \bar{y},(\bar{x} y \bar{x} \bar{y})^{3}$

Diam $X=14, \quad$ Girth $X=12$

$\mid$ Aut $X \mid=4608=2^{9} \cdot 3^{2}$

$\mid(\text { Aut } X)_{u} \mid=12=2^{2} \cdot 3$

StabPart:

$\left(1^{1}, 3^{1}, 6^{1}, 12^{1}, 12^{2}, 12^{4}, 6^{2} 12^{5}, 6^{4} 12^{7}, 3^{1} 6^{9} 12^{8}, 3^{1} 6^{5} 12^{11}, 2^{2} 6^{9} 12^{4}\right.$, $\left.3^{1} 12^{3}, 1^{1} 6^{1} 12^{1}, 3^{1} 6^{1}, 3^{1}\right)$

$\left(1^{1}, 3^{1}, 6^{1}, 12^{1}, 12^{2}, 12^{4}, 4^{1} 6^{2} 12^{5}, 6^{4} 12^{7}, 2^{1} 6^{4} 12^{11}, 3^{1} 6^{5} 12^{11}, 1^{1} 4^{3} 6^{2} 12^{6}\right.$, $\left.3^{1} 12^{3}, 1^{1} 6^{3}, 3^{1} 6^{1}, 1^{1} 2^{1}\right)$

$|\mathrm{mET}|=1152$, solvable

\begin{tabular}{|c|c|}
\hline & \\
\hline & \\
\hline \multicolumn{2}{|c|}{$X=\mathbf{S 7 6 8 b}$} \\
\hline Cov & Cov \\
\hline Aut $X$ & Aut $X$ \\
\hline $\mathbb{Z}_{2}$ & $\mathbb{Z}_{2}^{2}$ \\
\hline S384 & F192a \\
\hline
\end{tabular}




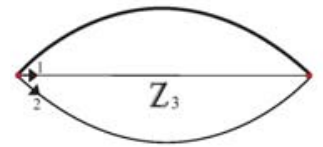

F2

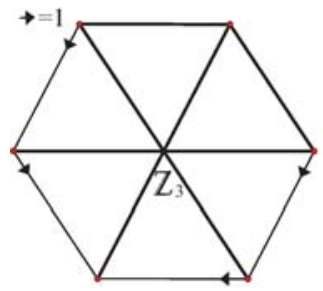

F6

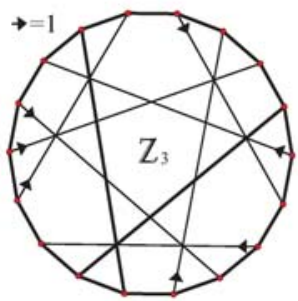

F18

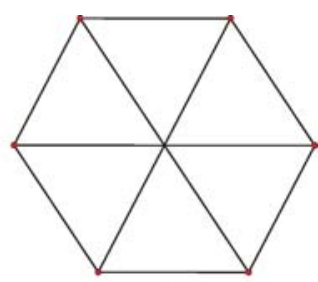

F6

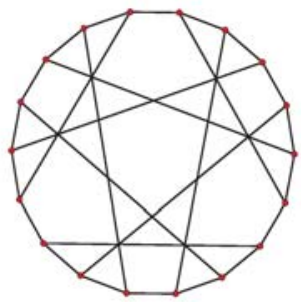

F18

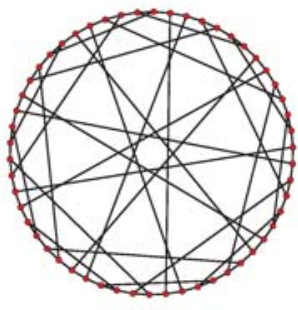

S54

Fig. 7 A sequence of three regular $\mathbb{Z}_{3}$-covers leading to the Gray graph S54

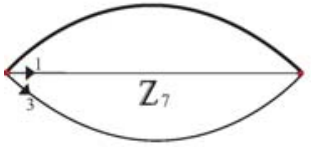

F2

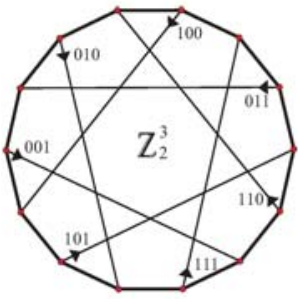

F14

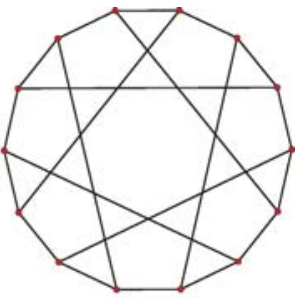

F14

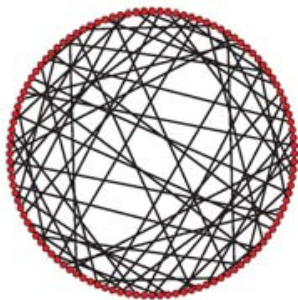

$\mathrm{S} 112$

Fig. 8 Construction of $\mathbf{S 1 1 2}$ via a regular $\mathbb{Z}_{7}$-cover of F2 and a regular $\mathbb{Z}_{2}^{3}$-cover of $\mathbf{F 1 4}$ 

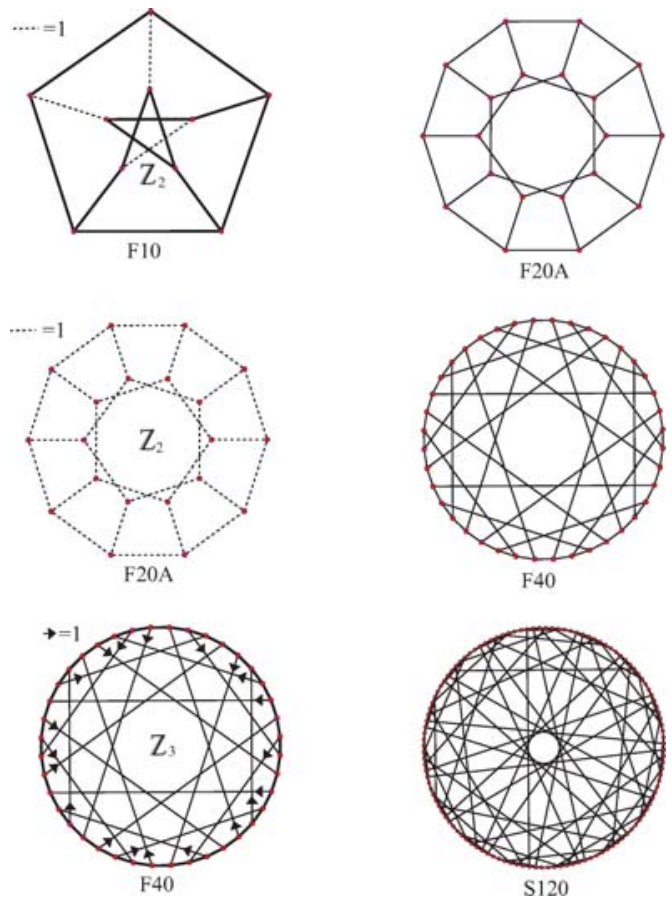

Fig. 9 A sequence of three regular cyclic covers leading to S120 via the dodecahedron F20A
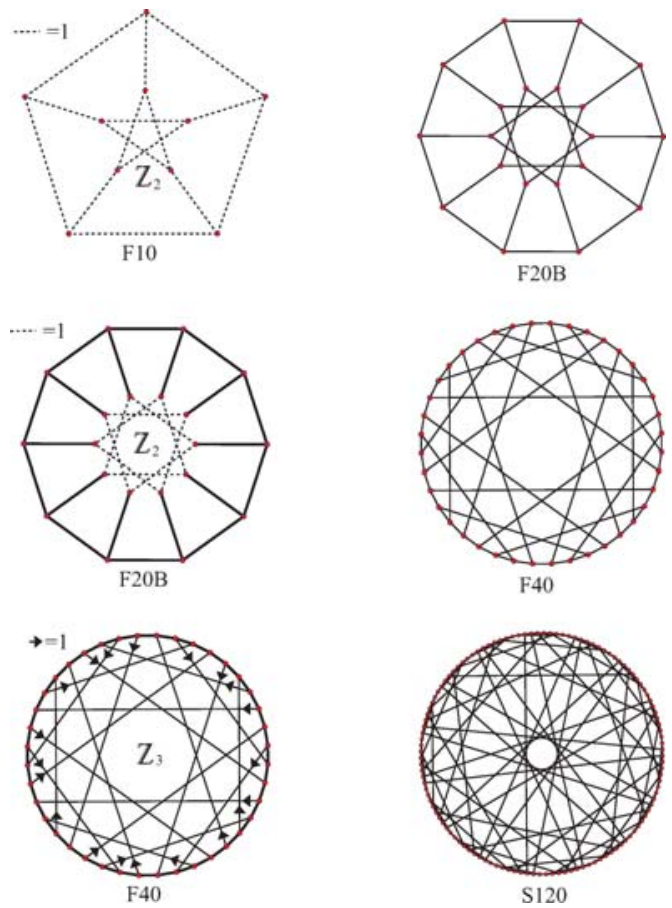

Fig. 10 A sequence of three regular cyclic covers leading to $\mathbf{S 1 2 0}$ via the Desargues graph F20B $\underline{\text { Springer }}$ 


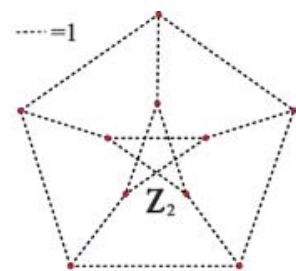

F10

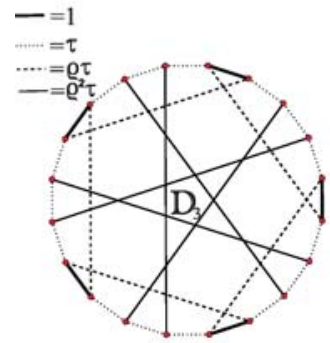

F20B

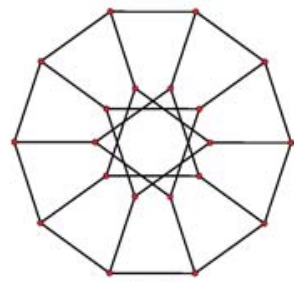

F20B

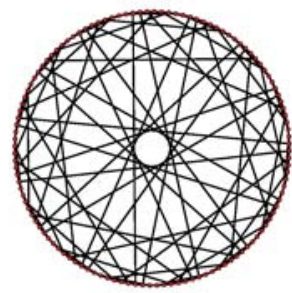

S120

Fig. 11 A voltage rule giving $\mathbf{S 1 2 0}$ as a regular (but indirect) $D_{3}$-cover of $\mathbf{F 2 0 B}$

\section{Voltage rules generating S54, S112 and S120}

In this final section we present five diagrams (see Figures 7, 8, 9, 10, 11) describing a voltage

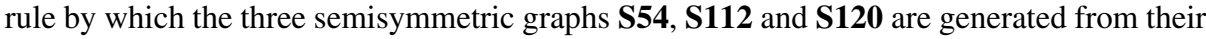
respective basic (and symmetric) counterparts: F2, F2, and the Petersen graph F10, via a sequence of direct elementary abelian covers; see also Figures 5, 6a and 6b. In the last case, two different routes may be taken, one leading through the dodecahedron F20a and the other through the Desargues graph F20b. Furthermore, a voltage rule is also described that gives the graph $\mathbf{S 1 2 0}$ as a regular (but indirect) $D_{3}$-cover of $\mathbf{F 2 0 b}$.

\section{References}

1. W. Bosma, C. Cannon, and C. Playoust, "The MAGMA algebra system I: The user language," J. Symbolic Comput. 24 (1997), 235-265.

2. I.Z. Bouwer, "An edge but not vertex transitive cubic graph," Bull. Can. Math. Soc. 11 (1968), 533-535.

3. I.Z. Bouwer, "On edge but not vertex transitive regular graphs," J. Combin. Theory, B 12 (1972), 32-40.

4. I.Z. Bouwer (ed.), The Foster Census, Charles Babbage Research Centre, Winnipeg, 1988.

5. M.D.E. Conder and P. Lorimer, "Automorphism Groups of Symmetric Graphs of Valency 3," J. Combin. Theory, Series B 47 (1989), 60-72.

6. M.D.E. Conder, P. Dobcsányi, B. Mc Kay and G. Royle, The Extended Foster Census, http: / /www.cs.uwa.edu.au/ gordon/remote/foster/.

7. M.D.E. Conder and P. Dobcsányi, "Trivalent symmetric graphs on up to 768 vertices," J. Combin. Math. Combin. Comput. 40 (2002), 41-63.

8. M.D.E. Conder, A. Malnič, D. Marušič, T. Pisanski and P. Potočnik, "The edge-transitive but not vertextransitive cubic graph on 112 vertices", J. Graph Theory 50 (2005), 25-42.

9. J.H. Conway, R.T. Curtis, S.P. Norton, R.A. Parker, and R.A. Wilson, Atlas of finite groups, Oxford University Press, Eynsham, 1985.

10. J.D. Dixon and B. Mortimer, Permutation Groups, Springer-Verlag, New York, 1996.

11. D.Ž. Djoković and G.L. Miller, "Regular groups of automorphisms of cubic graphs," J. Combin. Theory. B 29 (1980), 195-230.

12. P. Dobcsányi, Home Page, http://www. scitec.auckland.ac.nz/ peter. 
13. S.F. Du and D. Marušič, "Biprimitive graphs of smallest order," J. Algebraic Combin. 9 (1999), 151-156.

14. S.F. Du and M.Y. Xu, "A classification of semisymmetric graphs of order $2 p q$ (I)," Comm. Algebra 28 (2000), 2685-2715. J. Combin. Theory, Series B 29 (1980), 195-230.

15. J. Folkman, "Regular line-symmetric graphs," J. Combin. Theory 3 (1967), 215-232.

16. R. Frucht, "A canonical representation of trivalent Hamiltonian graphs," J. Graph Theory 1 (1977), 45-60.

17. M. Giudici, C.H. Li and C.E. Praeger, "Characterising finite locally $s$-arc transitive graphs with a star normal quotient," preprint.

18. C. Godsil, "On the full automorphism group of Cayley graphs," Combinatorica 1 (1981), 143-156.

19. C. Godsil and G. Royle, Algebraic Graph Theory, Graduate Texts in Mathematics 207, Springer-Verlag, New York, 2001.

20. D. Goldschmidt, “Automorphisms of trivalent graphs," Ann. Math. 111 (1980), 377-406.

21. D. Gorenstein, Finite Groups, Harper and Row, New York, 1968.

22. D. Gorenstein, Finite Simple Groups: An Introduction To Their Classification, Plenum Press, New York, 1982.

23. J.L. Gross and T.W. Tucker, Topological Graph Theory, Wiley-Interscience, New York, 1987.

24. M.E. Iofinova and A.A. Ivanov, Biprimitive cubic graphs, Investigations in Algebraic Theory of Combinatorial Objects (Proceedings of the seminar, Institute for System Studies, Moscow, 1985) Kluwer Academic Publishers, London, 1994, pp 459-472.

25. A.V. Ivanov, "On edge but not vertex transitive regular graphs," Ann. Discrete Math. 34 (1987), $273-286$.

26. M.H. Klin, "On edge but not vertex transitive graphs," Coll. Math. Soc. J. Bolyai, (25. Algebraic Methods in Graph Theory, Szeged, 1978), Budapest, 1981, 399-403.

27. F. Lazebnik and R. Viglione, "An infinite series of regular edge- but not vertex-transitive graphs," J. Graph Theory 41 (2002), 249-258.

28. S. Lipschutz and M.Y. Xu, "Note on infinite families of trivalent semisymmetric graphs," European J. Combin. 23 (2002), 707-711.

29. A. Malnič, D. Marušič and P. Potočnik, "On cubic graphs admitting an edge-transitive solvable group", J. Algebraic Combinatorics 20 (2004), 99-113.

30. A. Malnič, D. Marušič, P. Potočnik and C.Q. Wang, "An infinite family of cubic edge- but not vertextransitive graphs", Discrete Mathematics 280 (2004), 133-148.

31. A. Malnič, D. Marušič and P. Potočnik, "Elementary abelian covers of graphs", J. Algebraic Combinatorics 20 (2004), 71-97.

32. A. Malnič, R. Nedela, and M. Škoviera, "Lifting graph automorphisms by voltage assignments," European J. Combin. 21 (2000), 927-947.

33. D. Marušič, "Constructing cubic edge- but not vertex-transitive graphs," J. Graph Theory 35 (2000), 152-160.

34. D. Marušič and T. Pisanski, "The Gray graph revisited," J. Graph Theory 35 (2000), 1-7.

35. D. Marušič and P. Potočnik, "Semisymmetry of generalized Folkman graphs," European J. Combin. 22 (2001), 333-349.

36. M. Śkoviera, "A contribution to the theory of voltage graphs," Discrete Math. 61 (1986), 281-292.

37. W.T. Tutte, "A family of cubical graphs," Proc. Cambridge Phil. Soc. 43 (1948), 459-474.

38. H. Wielandt, Finite Permutation Groups, Academic Press, New York-London, 1964.

39. S.E. Wilson, “A worthy family of semisymmetric graphs", DiscreteMath. 271 (2003), 283-294. 